Supporting Information

\title{
Stereoselective Synthesis of cis-2-Ene-1,4-Diones via Aerobic Oxidation of Substituted Furans Catalyzed by $\mathrm{ABNO} / \mathrm{HNO}_{3}$
}

Liqun Yang, Jingyang Wang, Yue Wang, Xiaotong Li, Wei Liu, Zhaoguo Zhang*⿰† and Xiaomin $\mathrm{Xie}^{* \dagger}$

${ }^{\dagger}$ Shanghai Key Laboratory for Molecular Engineering of Chiral Drugs, School of Chemistry and Chemical Engineering, Shanghai Jiao Tong University, 800 Dongchuan Road, Shanghai 200240, China

"State Key Laboratory of Organometallic Chemistry, Shanghai Institute of Organic Chemistry, Chinese Academy of Sciences, 345 Lingling Road, Shanghai 200032, 


\section{Table of Contents}

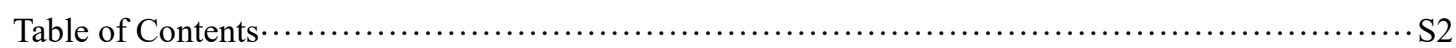

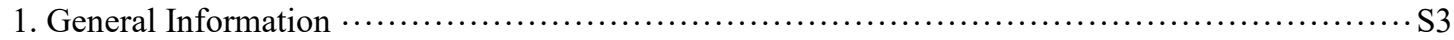

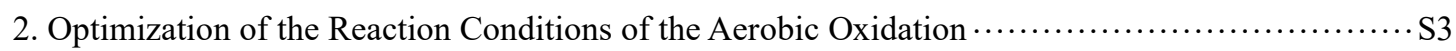

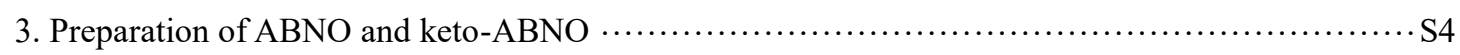

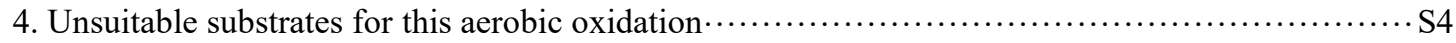

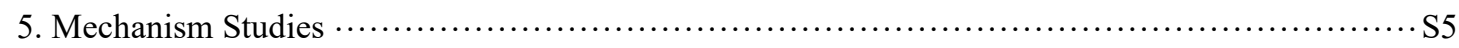

References $\quad$ S5

${ }^{1} \mathrm{H},{ }^{13} \mathrm{C},{ }^{19} \mathrm{~F}$ NMR Spectrum Copies $\quad$ S6 


\section{General Information}

Unless otherwise noted, all oxidation reactions were carried out under an atmosphere of $\mathrm{O}_{2}$ balloon. Materials were purchased from commercial suppliers and used without further purification. ${ }^{1} \mathrm{H}$ NMR, ${ }^{13} \mathrm{C}$ NMR and ${ }^{19} \mathrm{~F}$ NMR spectra were recorded on $400 \mathrm{MHz}, 500 \mathrm{MHz}$ or $600 \mathrm{MHz}$ spectrometers. The chemical shifts for ${ }^{1} \mathrm{H}$ NMR were recorded in ppm downfield from tetramethylsilane (TMS) with the solvent resonance as the internal standard. The chemical shifts for ${ }^{13} \mathrm{C}$ NMR were recorded in ppm downfield using the central peak of deuterochloroform $(77.16 \mathrm{ppm})$ as the internal standard. Coupling constants $(J)$ are reported in $\mathrm{Hz}$ and refer to apparent peak multiplications. HRMS were obtained on an ESI-TOF mass spectrometer. Flash column chromatography was performed on silica gel.

\section{Optimization of the Reaction Conditions of the Aerobic Oxidation}

Table S1. General screen of reaction conditions $\mathbf{s}^{\mathrm{a}}$

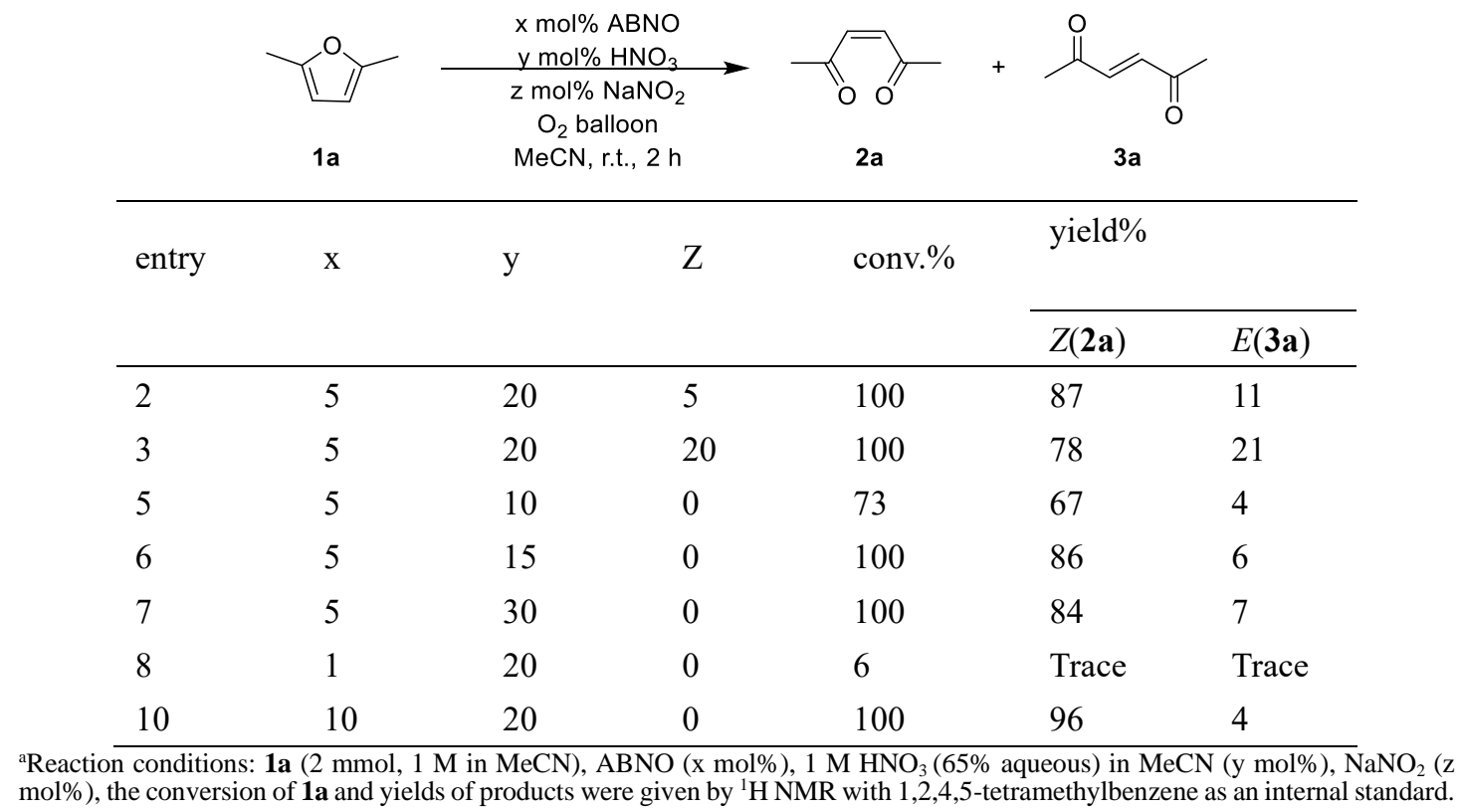


Figure S1. The profiles for the aerobic oxidation of 2,5-dimethylfuran
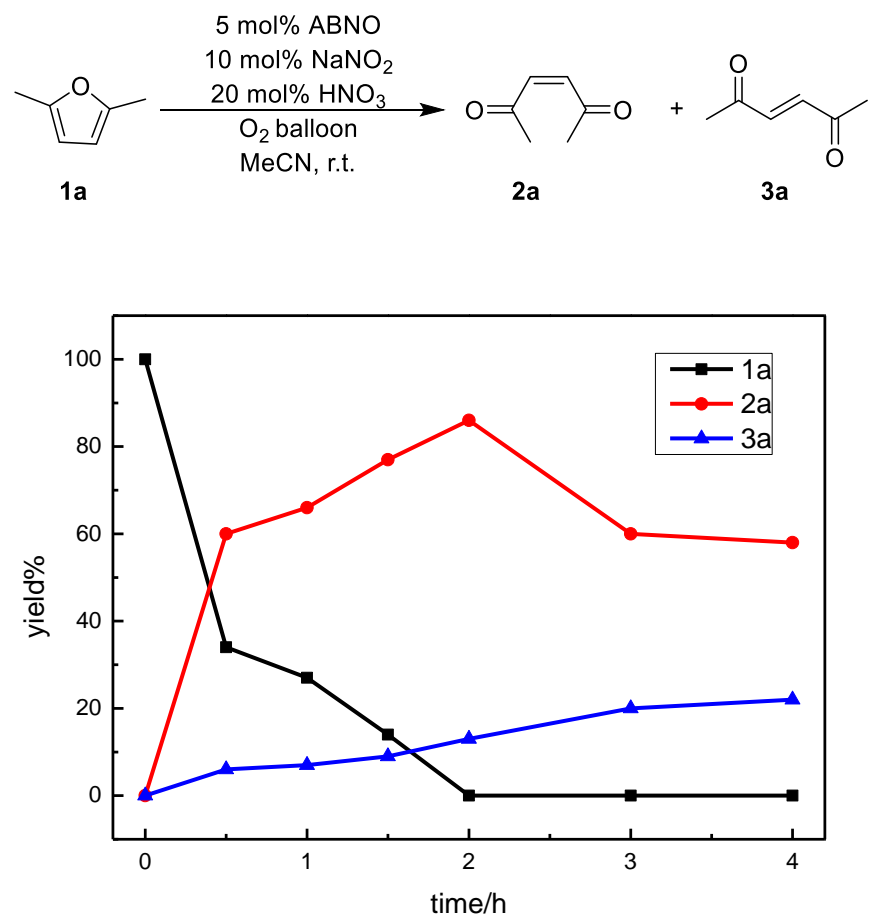

\section{Preparation of ABNO and keto-ABNO}
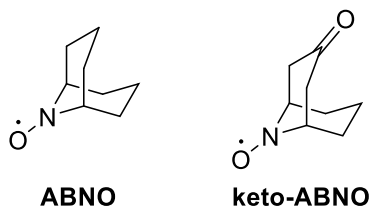

Synthesis according to the literature reporting. ${ }^{1}$

ABNO: Red solid. Purified by flash column chromatography (300-400 mesh silica gel, PE:EA=1:1).

HRMS-ESI (m/z): Calculated for $\mathrm{C}_{8} \mathrm{H}_{15} \mathrm{NO}(\mathrm{M}+\mathrm{H})^{+}: 141.1148$, Found: 141.1142 .

keto-ABNO: Yellow solid. Purified by flash column chromatography (300-400 mesh silica gel, PE:EA $=1: 1)$.

HRMS-ESI (m/z): Calculated for $\mathrm{C}_{8} \mathrm{H}_{14} \mathrm{NO}_{2}(\mathrm{M}+2 \mathrm{H})^{+}: 156.1019$, Found: 156.1023.

\section{Unsuitable substrates for this aerobic oxidation}<smiles>CC(=O)c1ccc(C)o1</smiles>

N.R.<smiles>O=C(O)c1ccc(C(=O)O)o1</smiles>

N.R.<smiles>CC(C)=CCc1ccc(C)o1</smiles>

N.R.

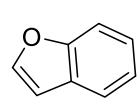

N.R.<smiles>Cc1ccc(CCCCC(=O)O)o1</smiles>

Trace<smiles>Cc1oc(C)c(C)c1C</smiles>

Trace<smiles>CC(=O)Cc1oc2c(c1C)C(=O)CCC2</smiles>

N.R. 


\section{Mechanism Studies}

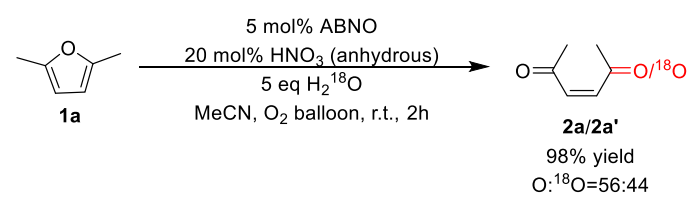

Step 1: Synthesis of anhydrous $\mathrm{HNO}_{3}{ }^{2}$ A mixture of nitric acid $(2 \mathrm{~mL})$ and sulfuric acid is distilled (2 $\mathrm{mL}$ ) twice to obtain anhydrous $\mathrm{HNO}_{3}$.

Step 2: Prepared $1 \mathrm{M} \mathrm{HNO}_{3}-\mathrm{MeCN}$ solution with anhydrous $\mathrm{MeCN}$ and anhydrous nitric acid prepared above. ABNO (7.0 mg, $0.05 \mathrm{mmol}, 5 \mathrm{~mol} \%$ ) was added into the reaction tube. After the tube was sealed with a rubber stopper, the atmoshpere was replaced to oxygen with oxygen balloons. The solution of $\mathrm{HNO}_{3}$ in $\left.\mathrm{CH}_{3} \mathrm{CN}(0.2 \mathrm{~mL}, 1 \mathrm{M} \mathrm{HNO})_{3}\right)$ and $\mathrm{H}_{2}{ }^{18} \mathrm{O}(90 \mathrm{uL}, 5 \mathrm{eq})$ were added to the tube by injection syringe, and white smoke was generated in the reaction system. After the solution of the substrate $\mathbf{1}$ in acetonitrile $(1 \mathrm{mmol}, 1 \mathrm{M})$ was added, the reaction mixture was stirred at room temperature for $2 \mathrm{~h}$. After the substituted furan was completely converted, the solvent was removed by rotary evaporator, and the residue was purified by fast column chromatography to obtain the target product. HRMS shows that about $44 \%$ of the products are ${ }^{18} \mathrm{O}$-labeled.

HRMS-ESI (m/z): Calculated for $\mathrm{C}_{6} \mathrm{H}_{9} \mathrm{O}^{18} \mathrm{O}(\mathrm{M}+\mathrm{H})^{+}: 115.0640$, Found: 115.0639 .

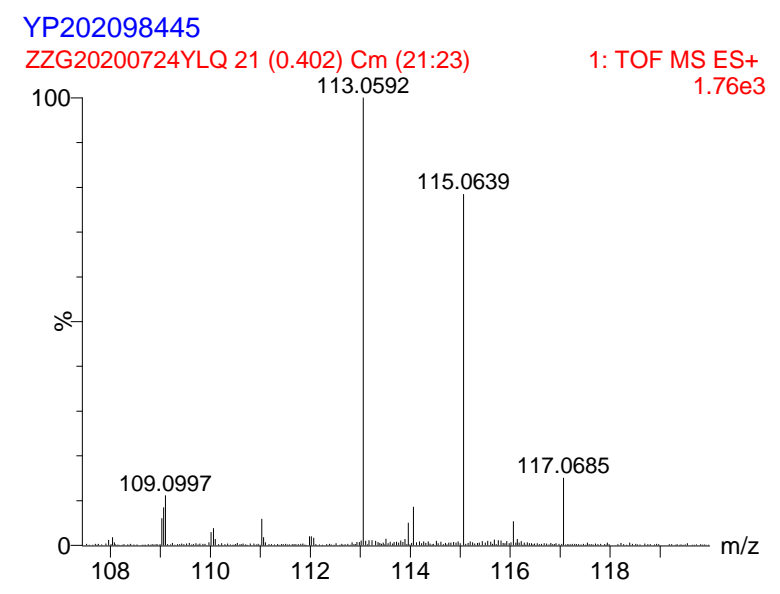

\section{References:}

(1) Shibuya, M.; Tomizawa, M.; Sasano, Y.; Iwabuchi, Y. An Expeditious Entry to 9Azabicyclo[3.3.1]nonane N-Oxyl (ABNO): Another Highly Active Organocatalyst for Oxidation of Alcohols. J. Org. Chem. 2009, 74, 4619-4622.

(2) Liang, P. Tetranitromethane. Org. Synth. 1941, 21, 105. 

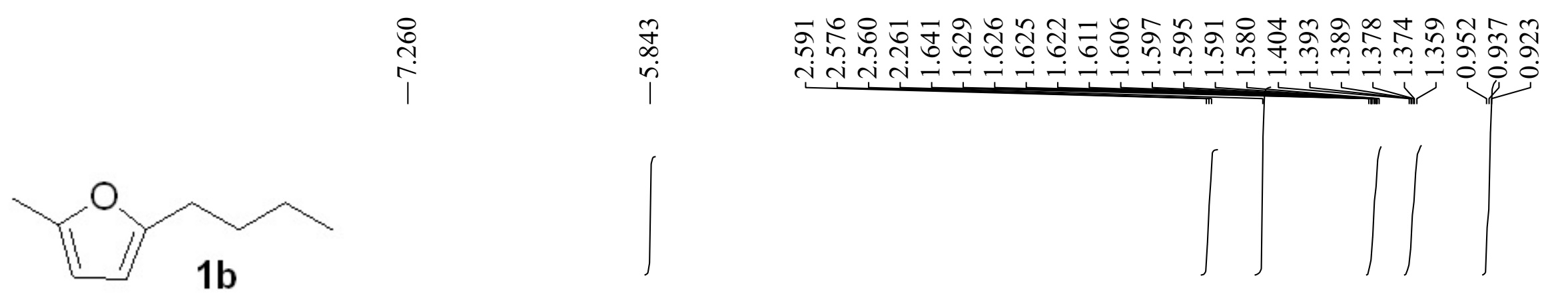

${ }^{1} \mathrm{HNMR}\left(500 \mathrm{MHz}, \mathrm{CDCl}_{3}\right)$

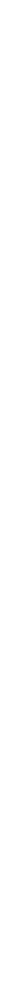




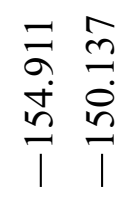

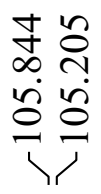

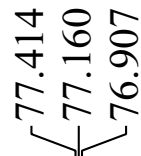

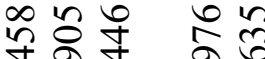

वंतส

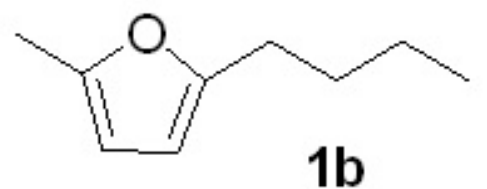

${ }^{13} \mathrm{C}\left\{{ }^{1} \mathrm{H}\right\} \mathrm{NMR}\left(101 \mathrm{MHz}, \mathrm{CDCl}_{3}\right)$

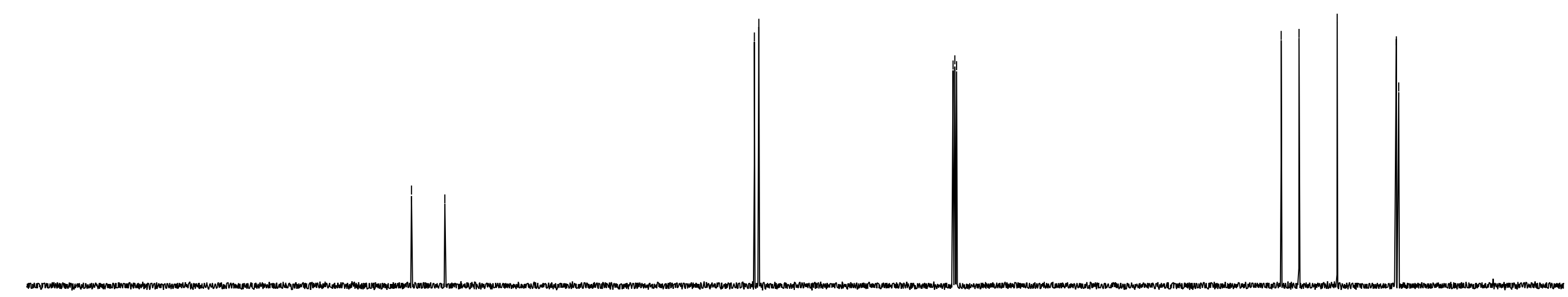

$\begin{array}{rllllllllllllllllllllllll}210 & 200 & 190 & 180 & 170 & 160 & 150 & 140 & 130 & 120 & 110 & 100 & 90 & 80 & 70 & 60 & 50 & 40 & 30 & 20 & 10 & 0 & -10\end{array}$




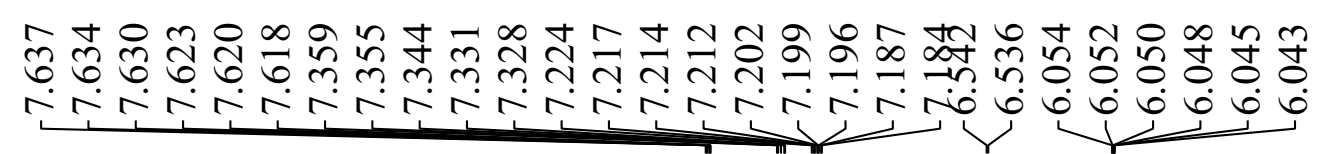

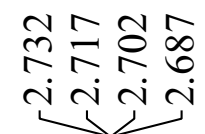

วิกำก

N

Et<smiles>Cc1ccc(-c2ccccc2)o1</smiles>

$1 \mathrm{c}$

${ }^{1} \mathrm{HNMR}\left(500 \mathrm{MHz}, \mathrm{CDCl}_{3}\right)$

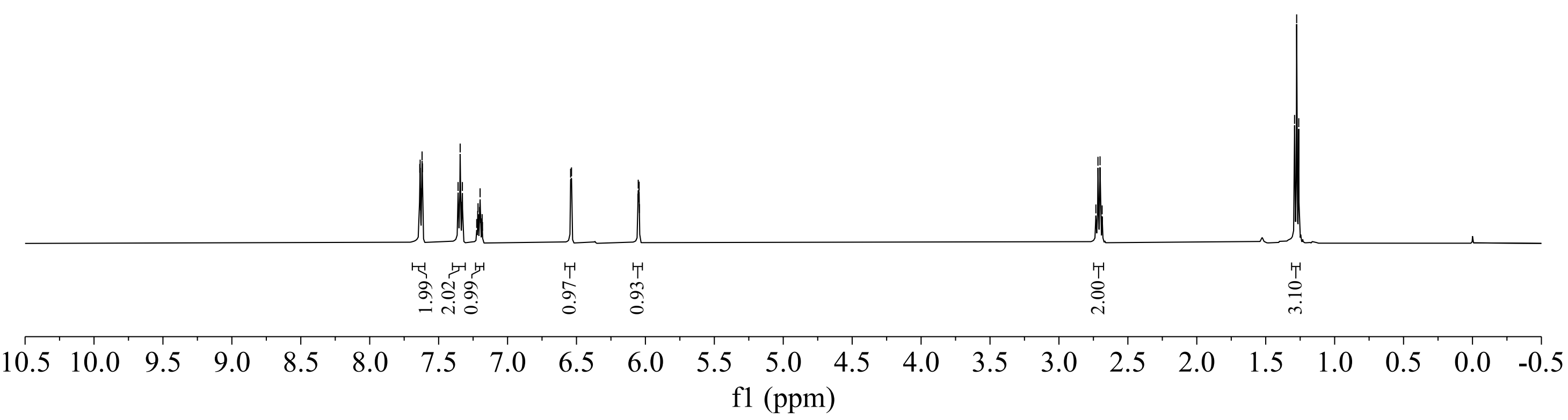



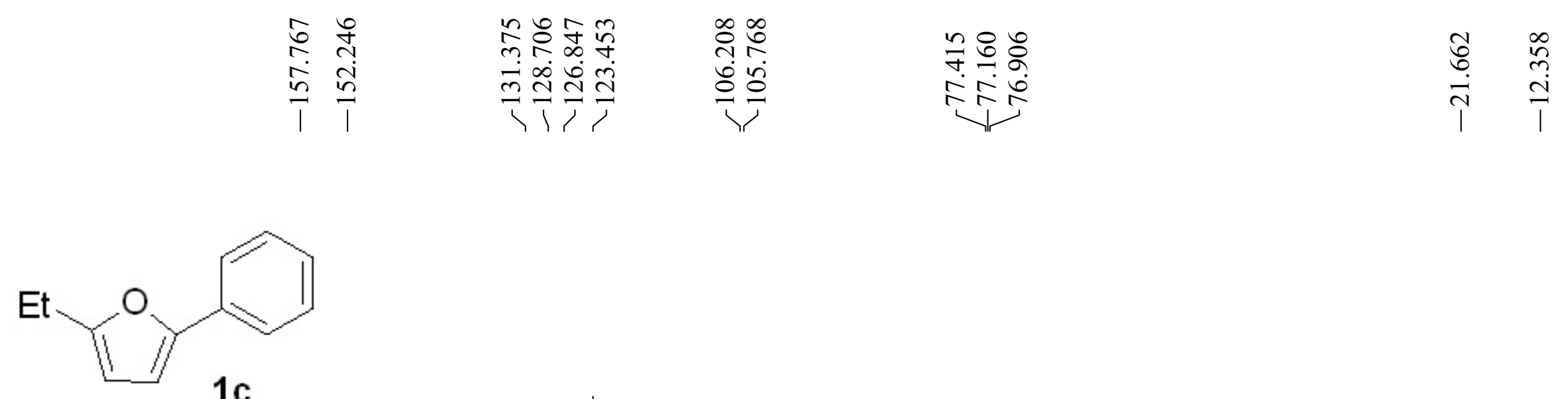

${ }^{13} \mathrm{C}\left\{{ }^{1} \mathrm{H}\right\}$ NMR $\left(126 \mathrm{MHz}, \mathrm{CDCl}_{3}\right)$
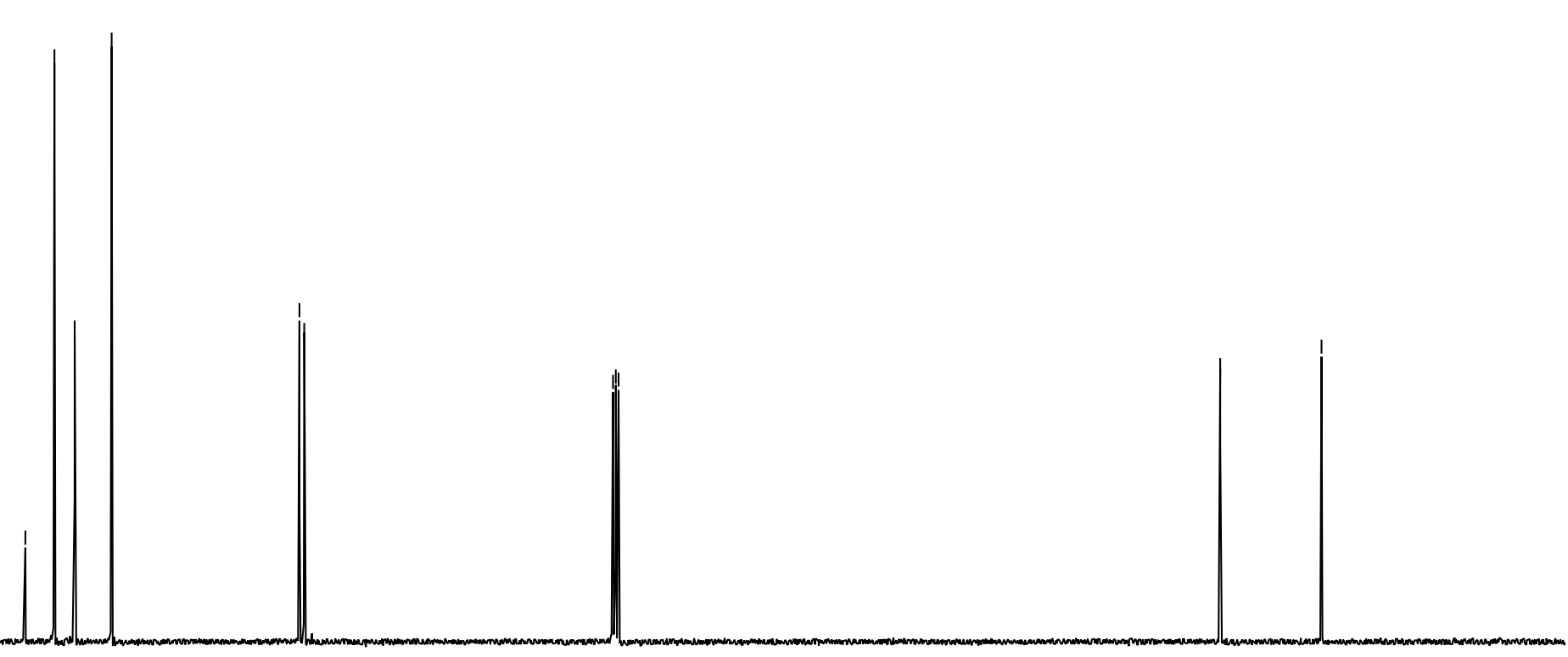

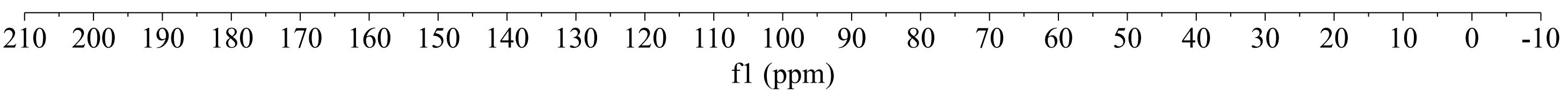



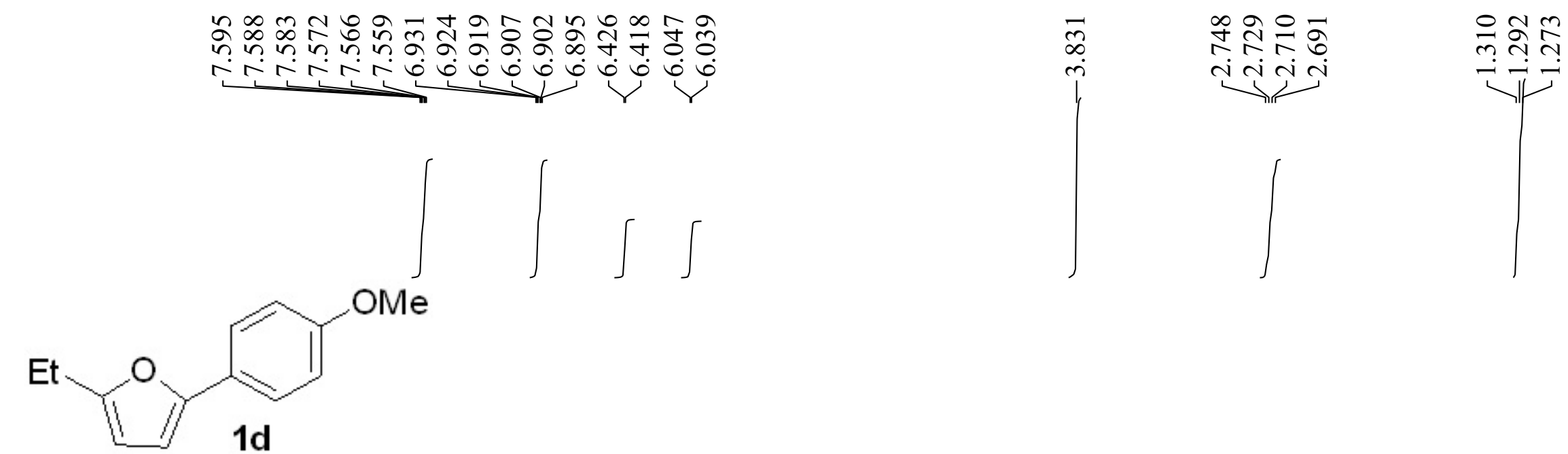

${ }^{1} \mathrm{H}$ NMR $\left(400 \mathrm{MHz}, \mathrm{CDCl}_{3}\right)$

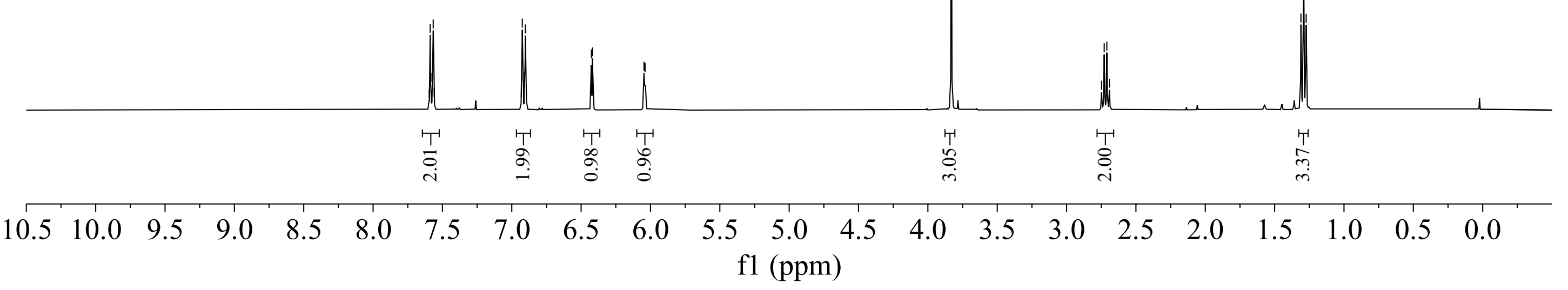




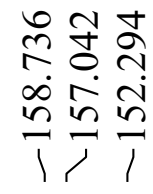

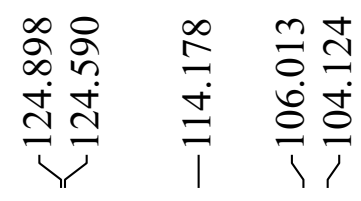

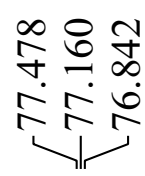
$\stackrel{\%}{\stackrel{7}{i}}$

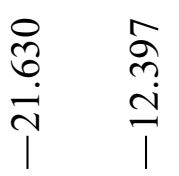

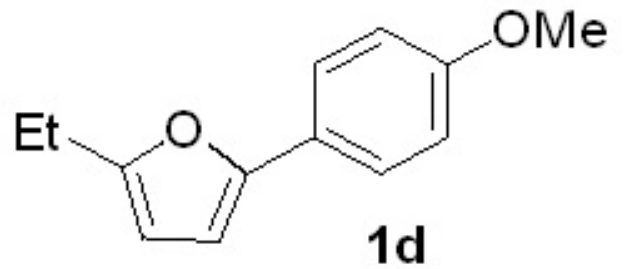

${ }^{13} \mathrm{C}\left\{{ }^{1} \mathrm{H}\right\}$ NMR $\left(101 \mathrm{MHz}, \mathrm{CDCl}_{3}\right)$

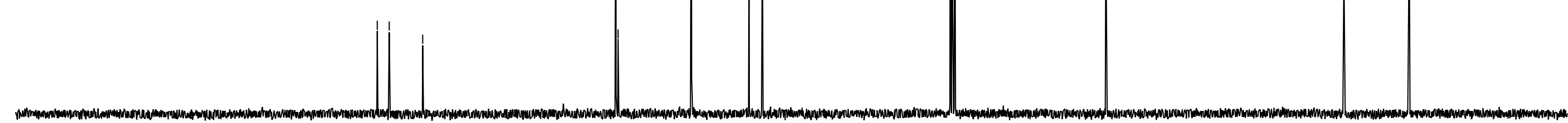

$\begin{array}{rlllllllllllllllllllllll}210 & 200 & 190 & 180 & 170 & 160 & 150 & 140 & 130 & 120 & 110 & 100 & 90 & 80 & 70 & 60 & 50 & 40 & 30 & 20 & 10 & 0 & -10 \\ \text { fl }(\mathrm{ppm})\end{array}$



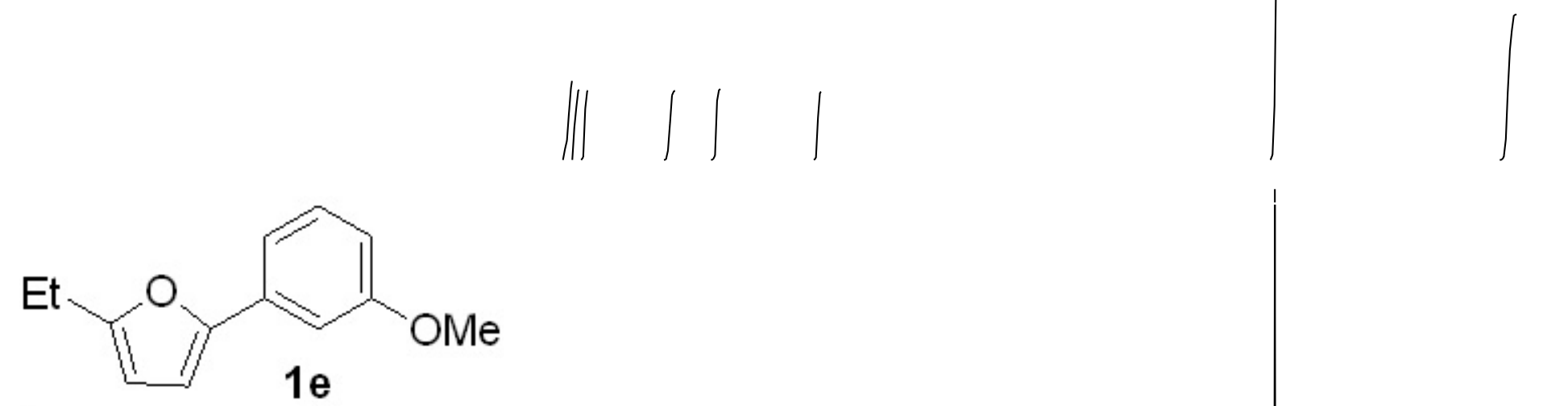

${ }^{1} \mathrm{H}$ NMR $\left(600 \mathrm{MHz}, \mathrm{CDCl}_{3}\right)$

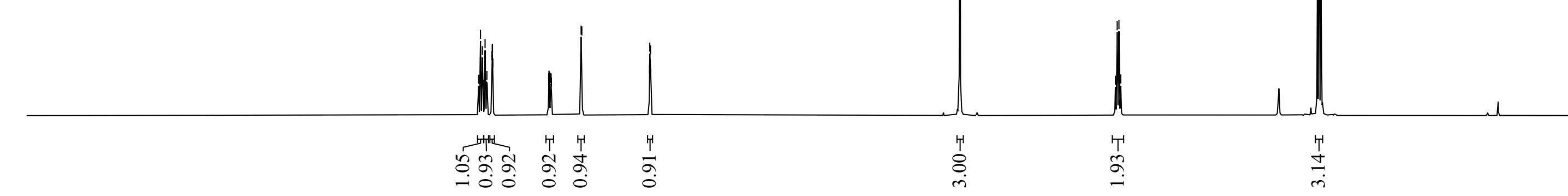

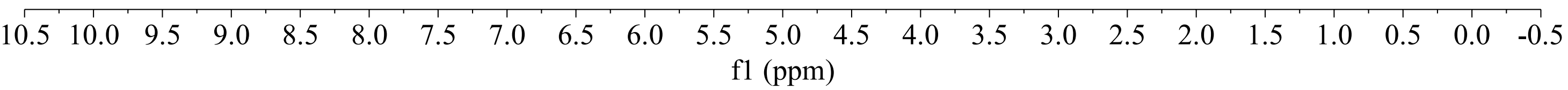




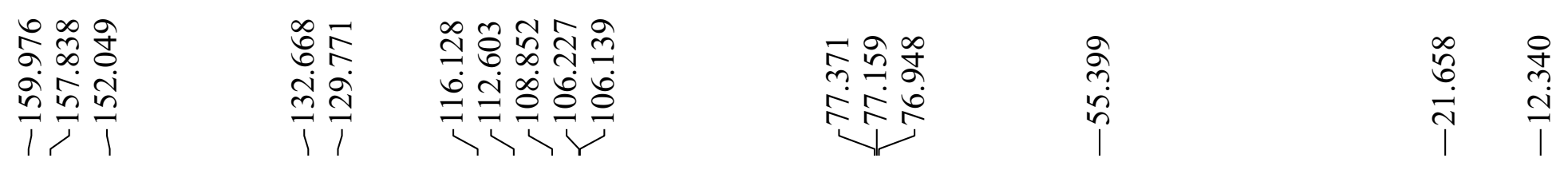

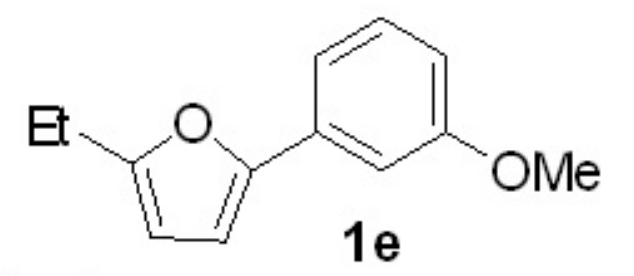

${ }^{13} \mathrm{C}\left\{{ }^{1} \mathrm{H}\right\} \operatorname{NMR}\left(151 \mathrm{MHz}, \mathrm{CDCl}_{3}\right)$

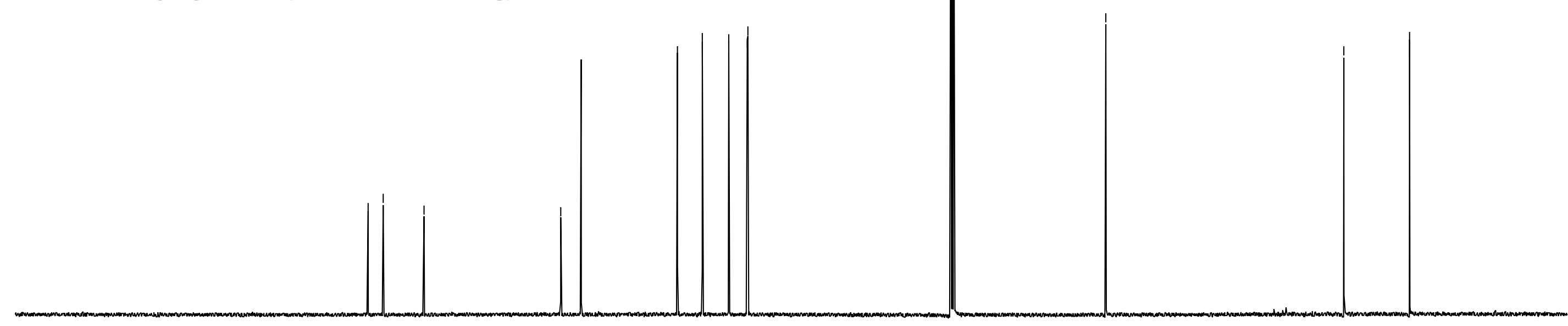

$\begin{array}{rlllllllllllllllllllllllll}210 & 200 & 190 & 180 & 170 & 160 & 150 & 140 & 130 & 120 & 110 & 100 & 90 & 80 & 70 & 60 & 50 & 40 & 30 & 20 & 10 & 0 & -10 \\ \mathrm{fl}(\mathrm{ppm})\end{array}$



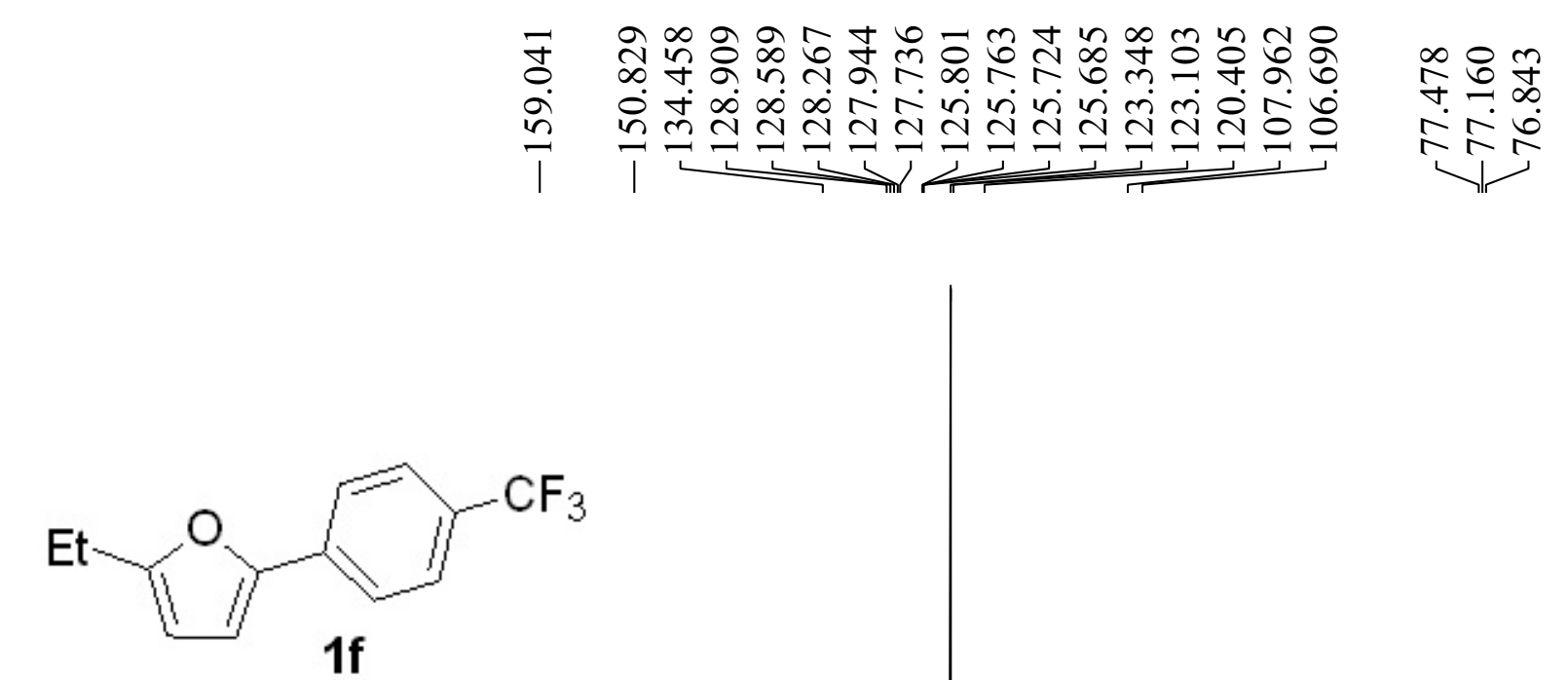

${ }^{13} \mathrm{C}\left\{{ }^{1} \mathrm{H}\right\}$ NMR $\left(101 \mathrm{MHz}, \mathrm{CDCl}_{3}\right)$

$\begin{array}{lllllllllllll}210 & 200 & 190 & 180 & 170 & 160 & 150 & 140 & 130 & 120 & 110 & 100 & 90\end{array}$ 


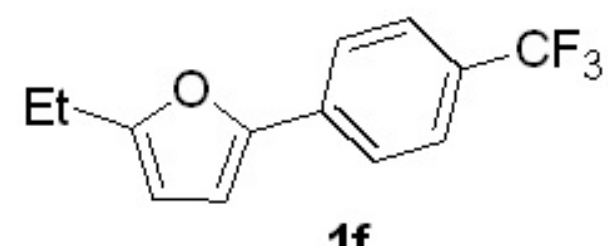

${ }^{19} \mathrm{~F}$ NMR $\left(376 \mathrm{MHz}, \mathrm{CDCl}_{3}\right)$

$$
\begin{aligned}
& \begin{array}{llllllllllllllllllllllllllllllll}
10 & 0 & -10 & -20 & -30 & -40 & -50 & -60 & -70 & -80 & -90 & -100 & -110 & -120 & -130 & -140 & -150 & -160 & -170 & -180 & -190 & -200 & -210
\end{array} \\
& \text { f1 ( } \mathrm{ppm})
\end{aligned}
$$




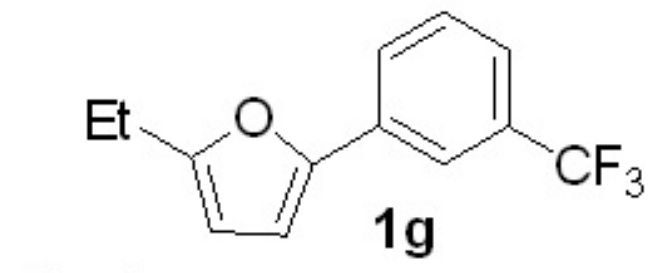

${ }^{13} \mathrm{C}\left\{{ }^{1} \mathrm{H}\right\} \operatorname{NMR}\left(151 \mathrm{MHz}, \mathrm{CDCl}_{3}\right)$

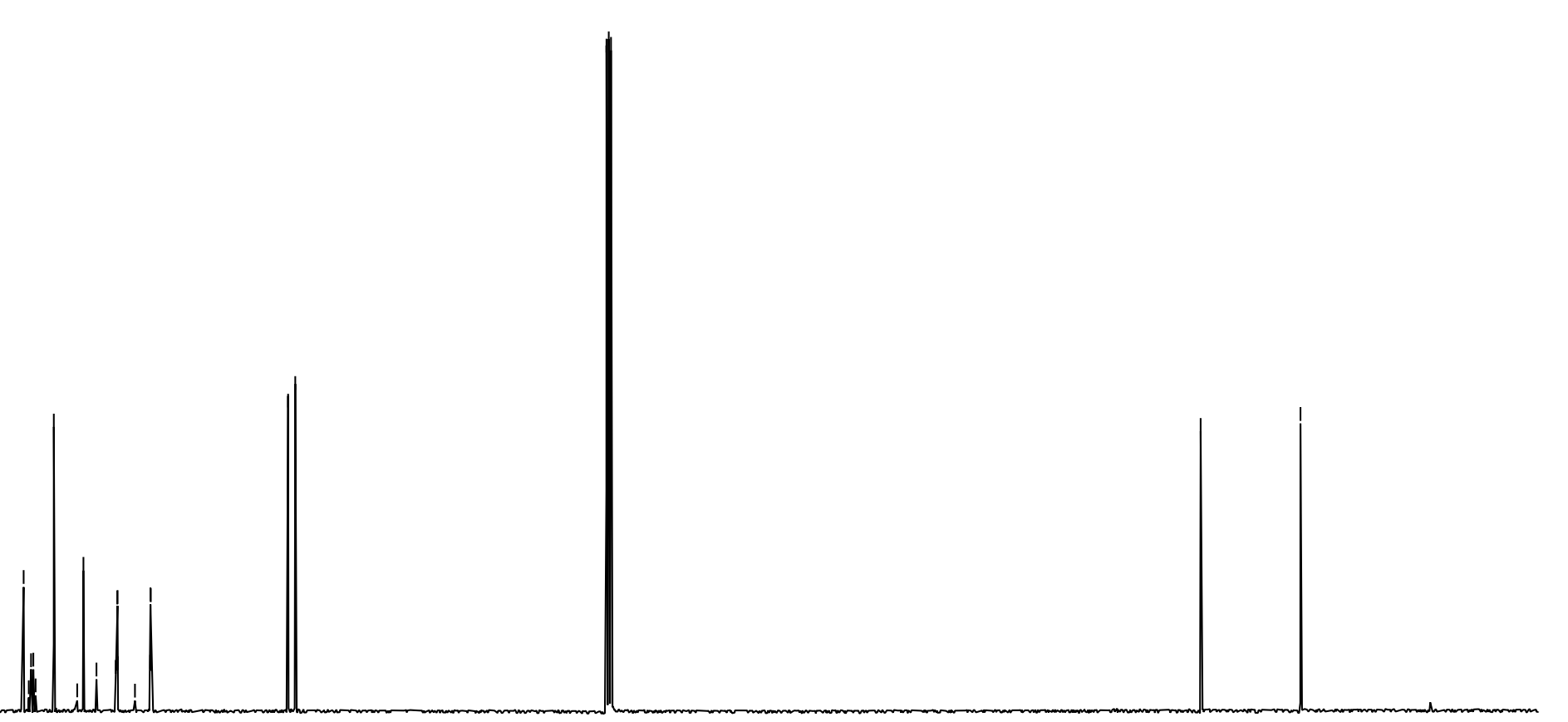

$\begin{array}{rllllllllllllllllllllll}210 & 200 & 190 & 180 & 170 & 160 & 150 & 140 & 130 & 120 & \begin{array}{c}110 \\ \mathrm{f} 1(\mathrm{ppm})\end{array} & 100 & 90 & 80 & 70 & 60 & 50 & 40 & 30 & 20 & 10 & 0 & -10\end{array}$




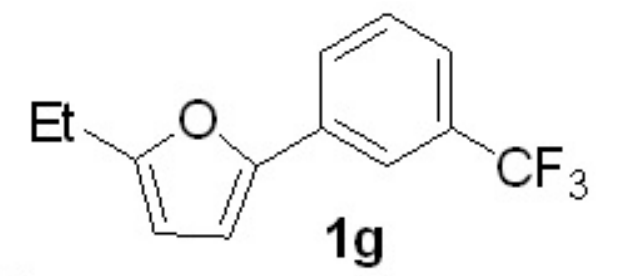

${ }^{19} \mathrm{~F}$ NMR (376 MHz, CDCl 3 )

$$
\begin{aligned}
& \begin{array}{llllllllllllllllllllllllll}
10 & 0 & -10 & -20 & -30 & -40 & -50 & -60 & -70 & -80 & -90 & -100 & -110 & -120 & -130 & -140 & -150 & -160 & -170 & -180 & -190 & -200 & -210
\end{array} \\
& \text { f1 (ppm) }
\end{aligned}
$$



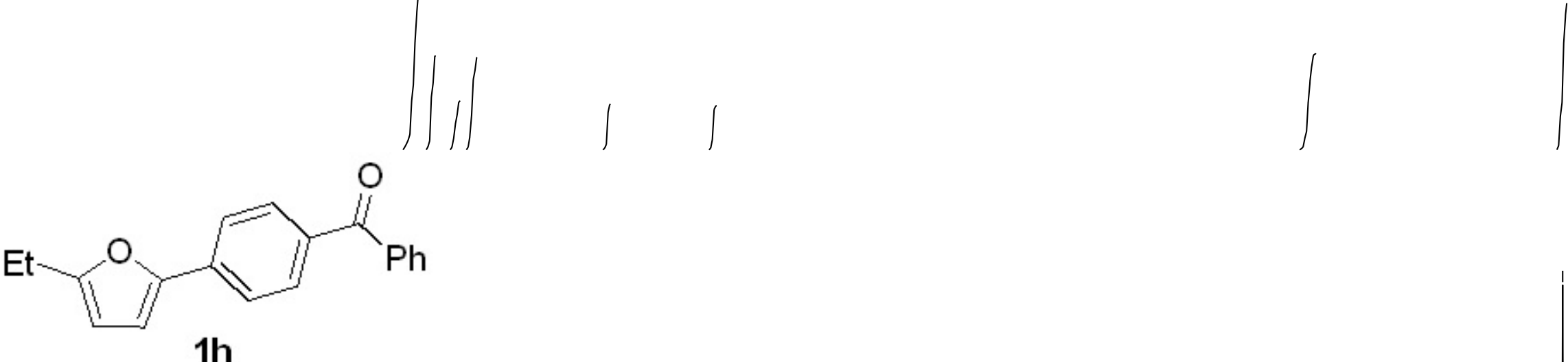

${ }^{1} \mathrm{H}$ NMR $\left(500 \mathrm{MHz}, \mathrm{CDCl}_{3}\right)$

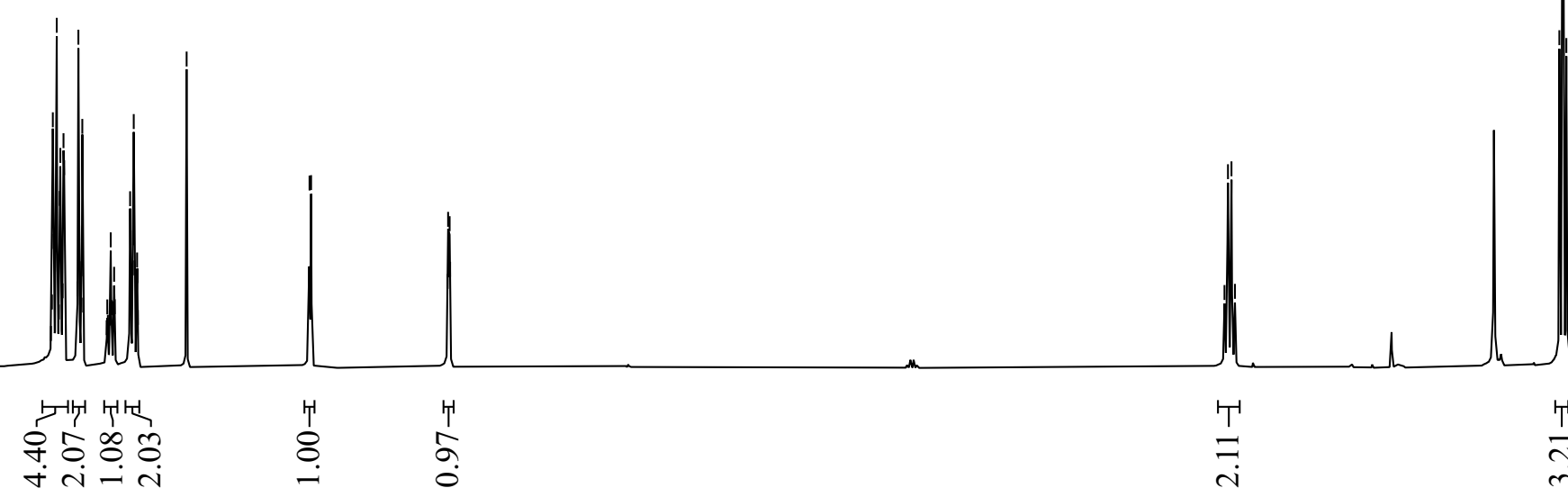

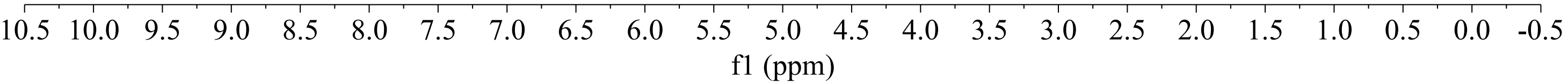



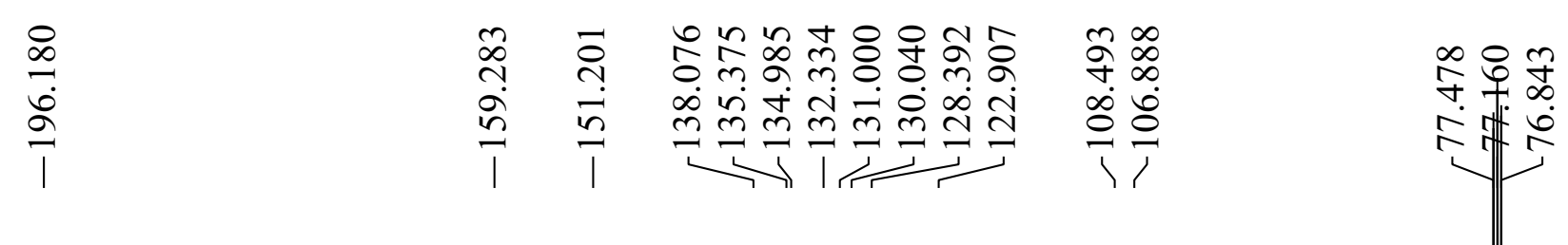

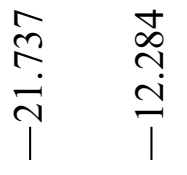

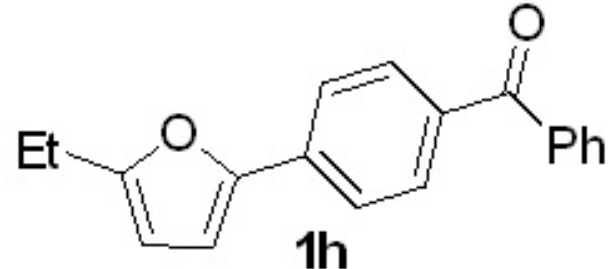

${ }^{13} \mathrm{C}\left\{{ }^{1} \mathrm{H}\right\}$ NMR $\left(101 \mathrm{MHz}, \mathrm{CDCl}_{3}\right)$

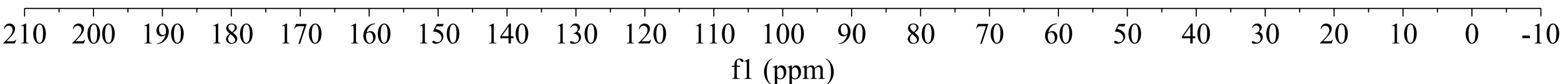




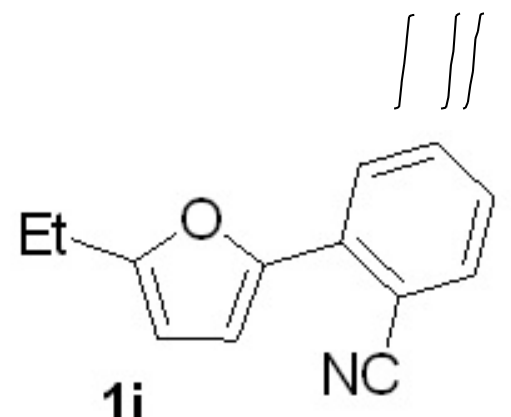

${ }^{1} \mathrm{H} \mathrm{NMR}\left(400 \mathrm{MHz}, \mathrm{CDCl}_{3}\right)$

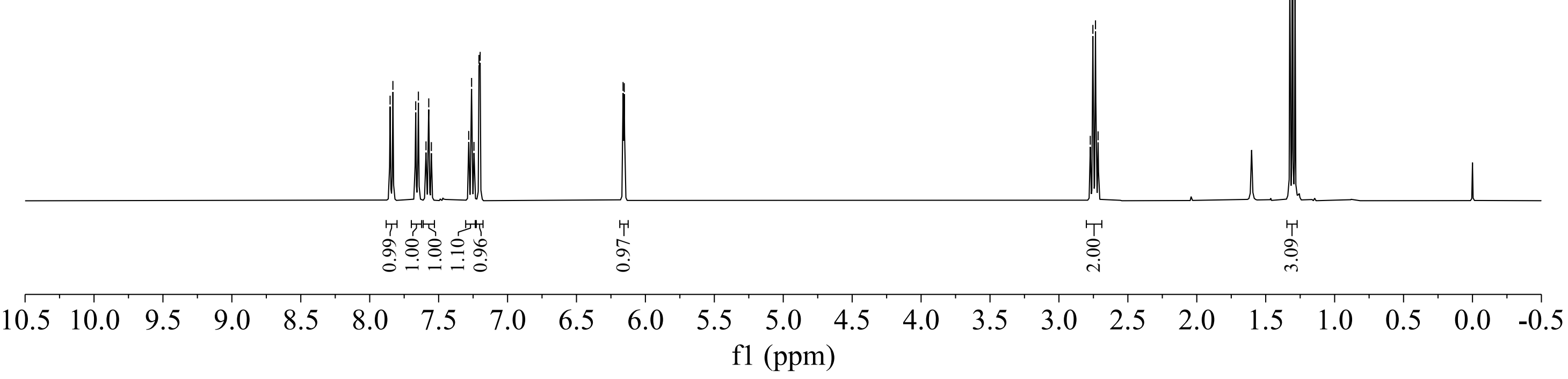




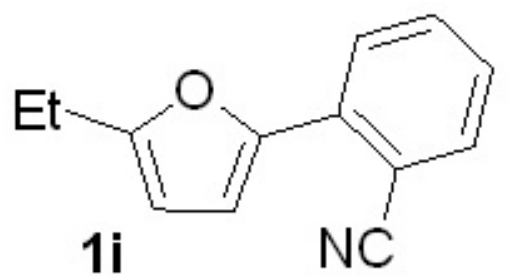

${ }^{13} \mathrm{C}\left\{{ }^{1} \mathrm{H}\right\}$ NMR $\left(101 \mathrm{MHz}, \mathrm{CDCl}_{3}\right)$

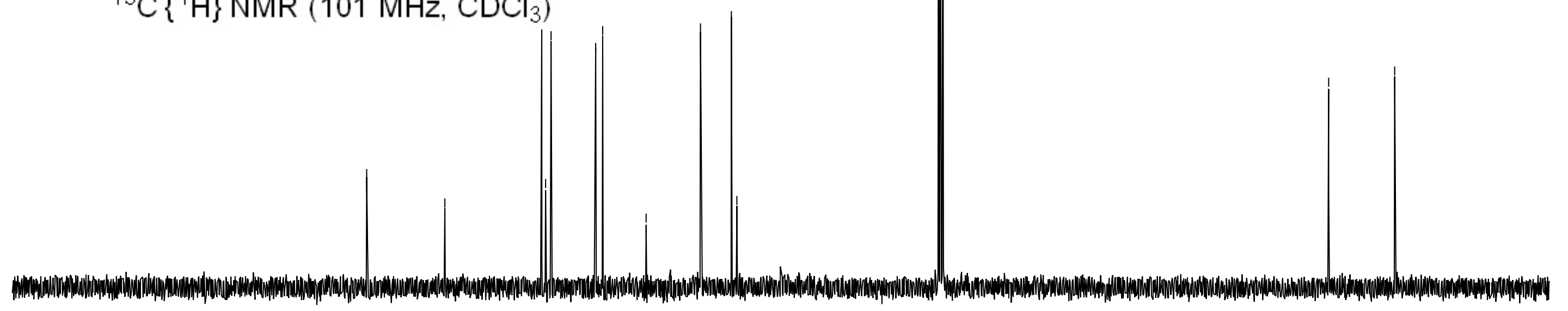

$\begin{array}{rlllllllllllllllllllllll}210 & 200 & 190 & 180 & 170 & 160 & 150 & 140 & 130 & 120 & 110 & 100 & 90 & 80 & 70 & 60 & 50 & 40 & 30 & 20 & 10 & 0 & -10 \\ \text { fl }(\mathrm{ppm})\end{array}$




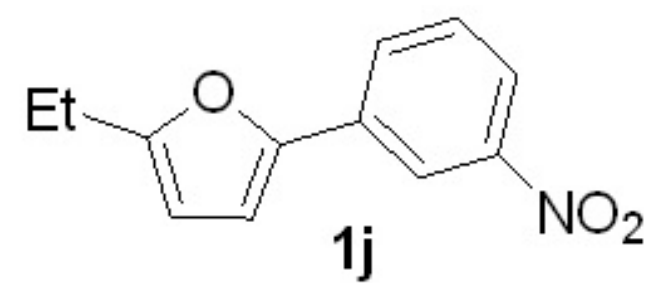

${ }^{1} \mathrm{H}$ NMR $\left(400 \mathrm{MHz}, \mathrm{CDCl}_{3}\right)$

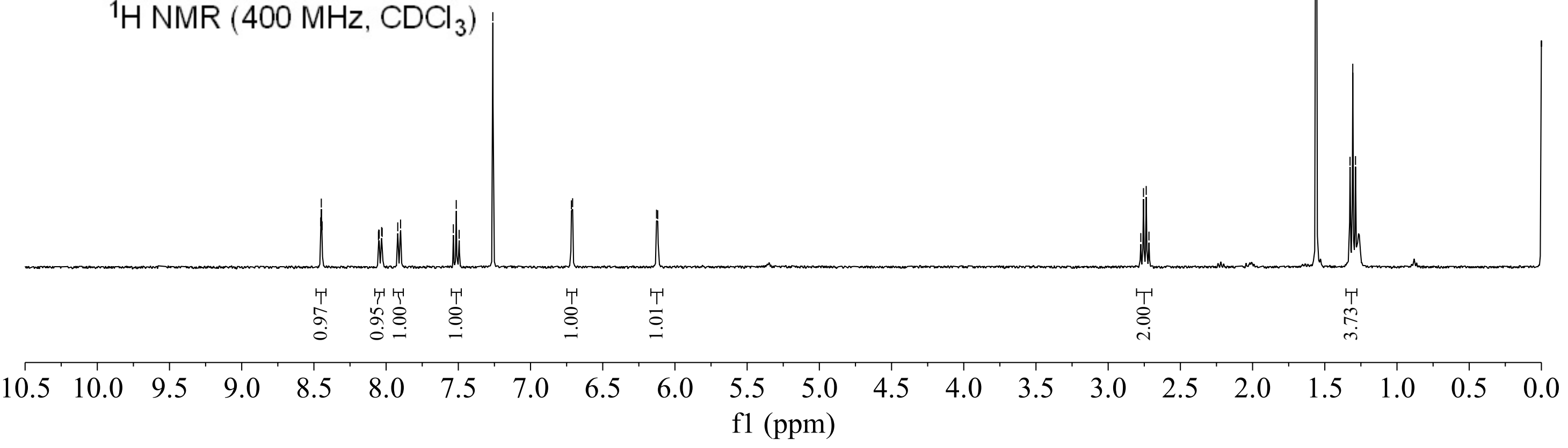



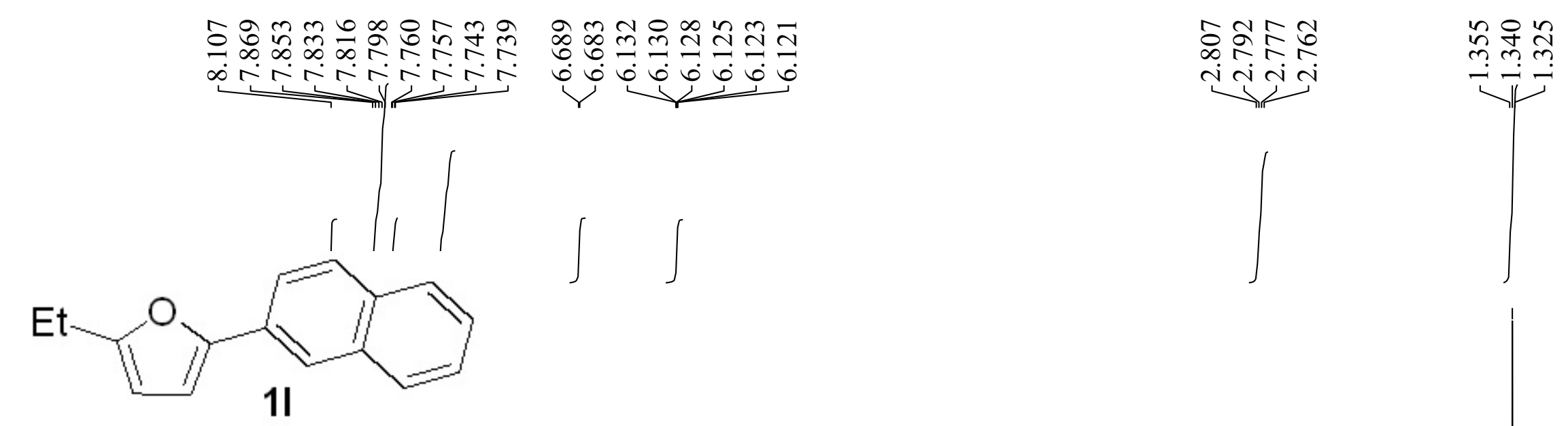

${ }^{1} \mathrm{H} \mathrm{NMR}\left(500 \mathrm{MHz}, \mathrm{CDCl}_{3}\right)$

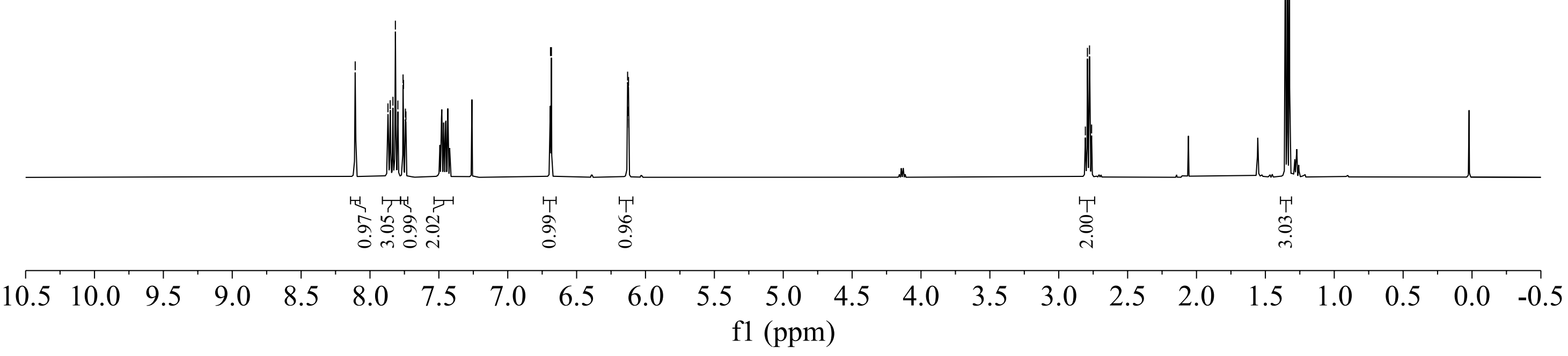




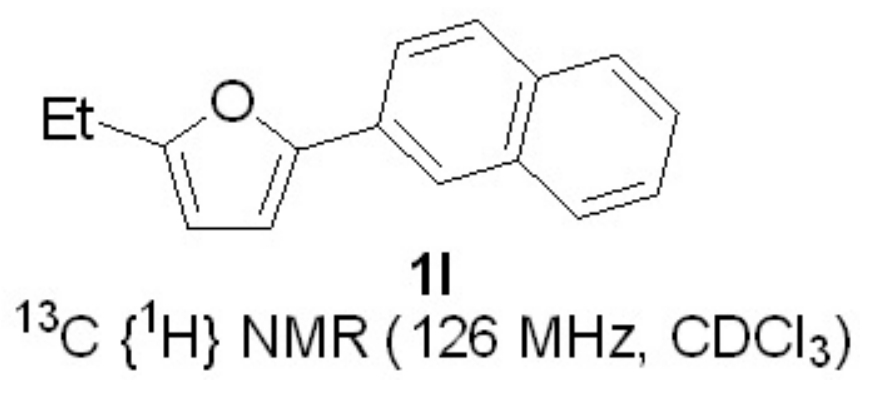

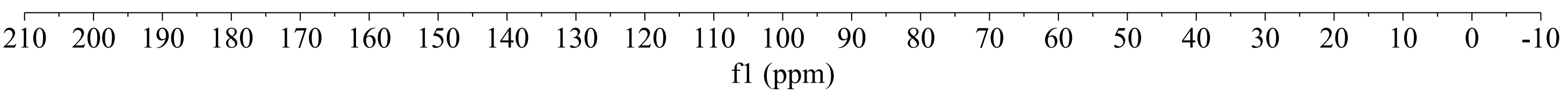




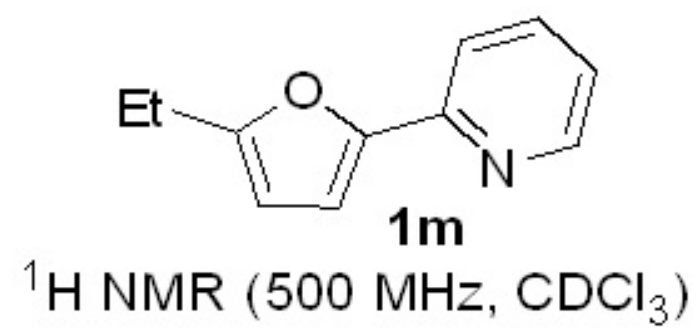

${ }^{1} \mathrm{H}$ NMR $\left(500 \mathrm{MHz}, \mathrm{CDCl}_{3}\right)$

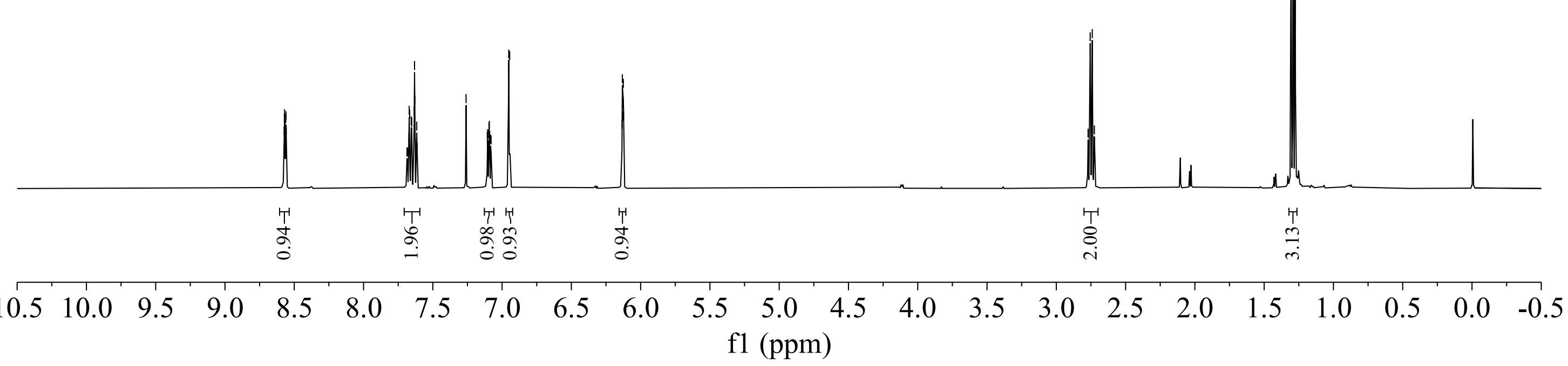




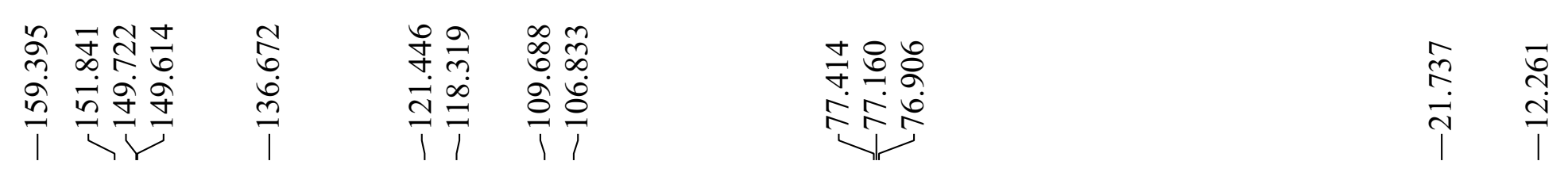

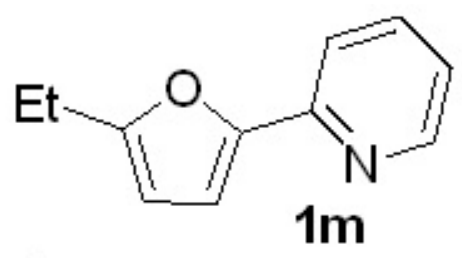

${ }^{13} \mathrm{C}\left\{{ }^{1} \mathrm{H}\right\} \mathrm{NMR}\left(126 \mathrm{MHz}, \mathrm{CDCl}_{3}\right)$

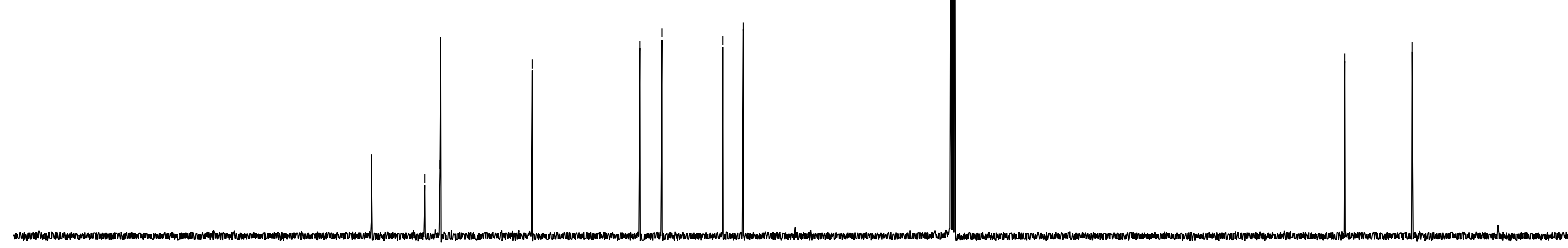

$\begin{array}{rllllllllllllllllllllllll}210 & 200 & 190 & 180 & 170 & 160 & 150 & 140 & 130 & 120 & 110 & 100 & 90 & 80 & 70 & 60 & 50 & 40 & 30 & 20 & 10 & 0 & -10\end{array}$



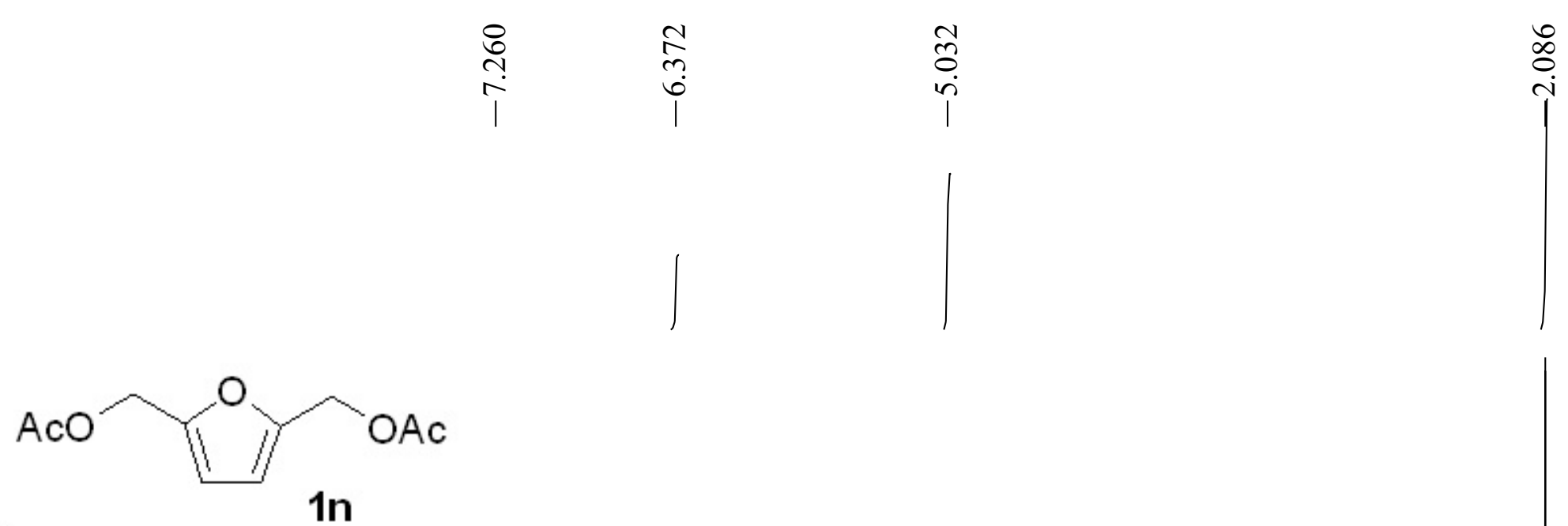

${ }^{1} \mathrm{H}$ NMR (400 MHz, CDCl 3 )
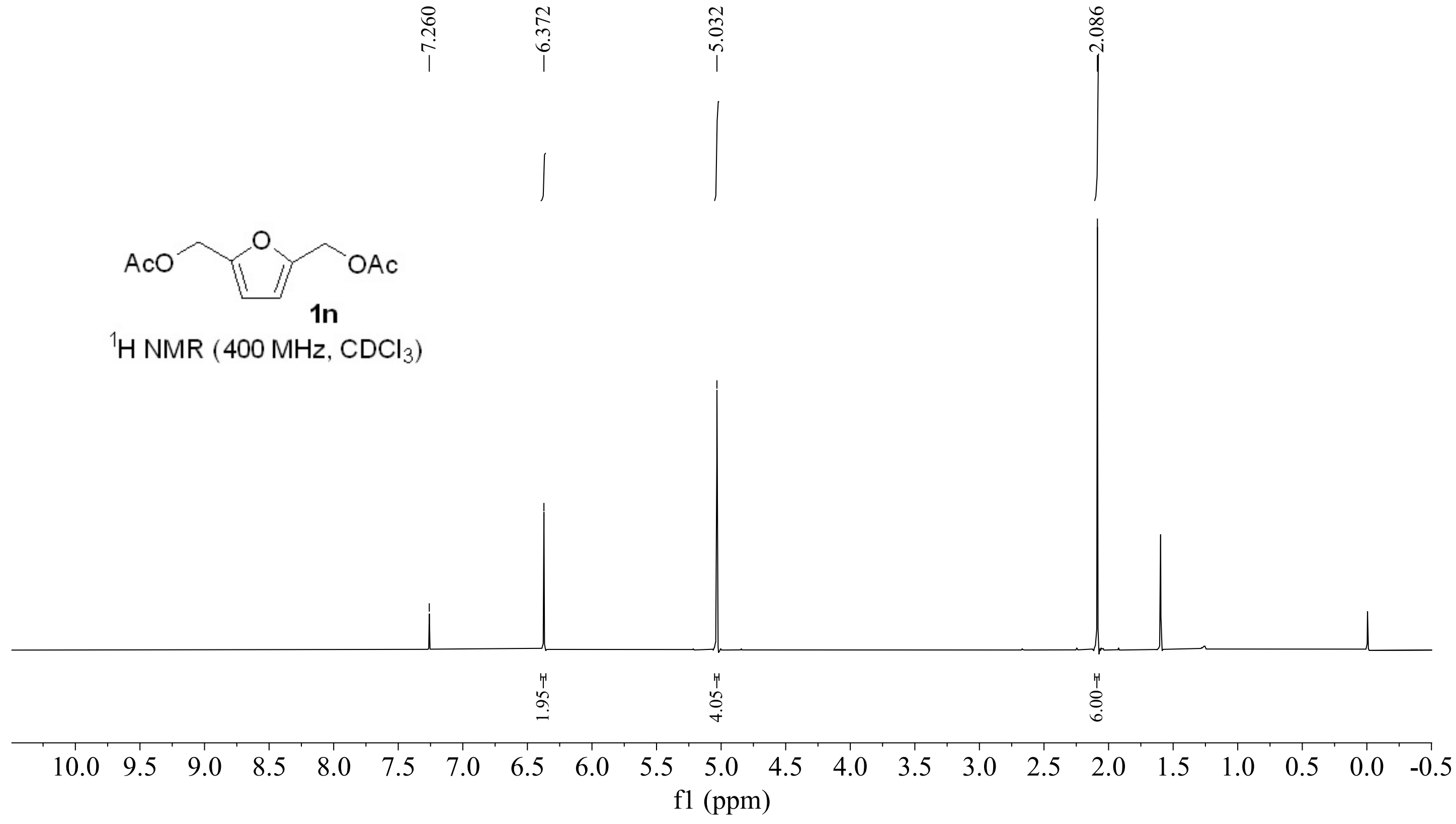


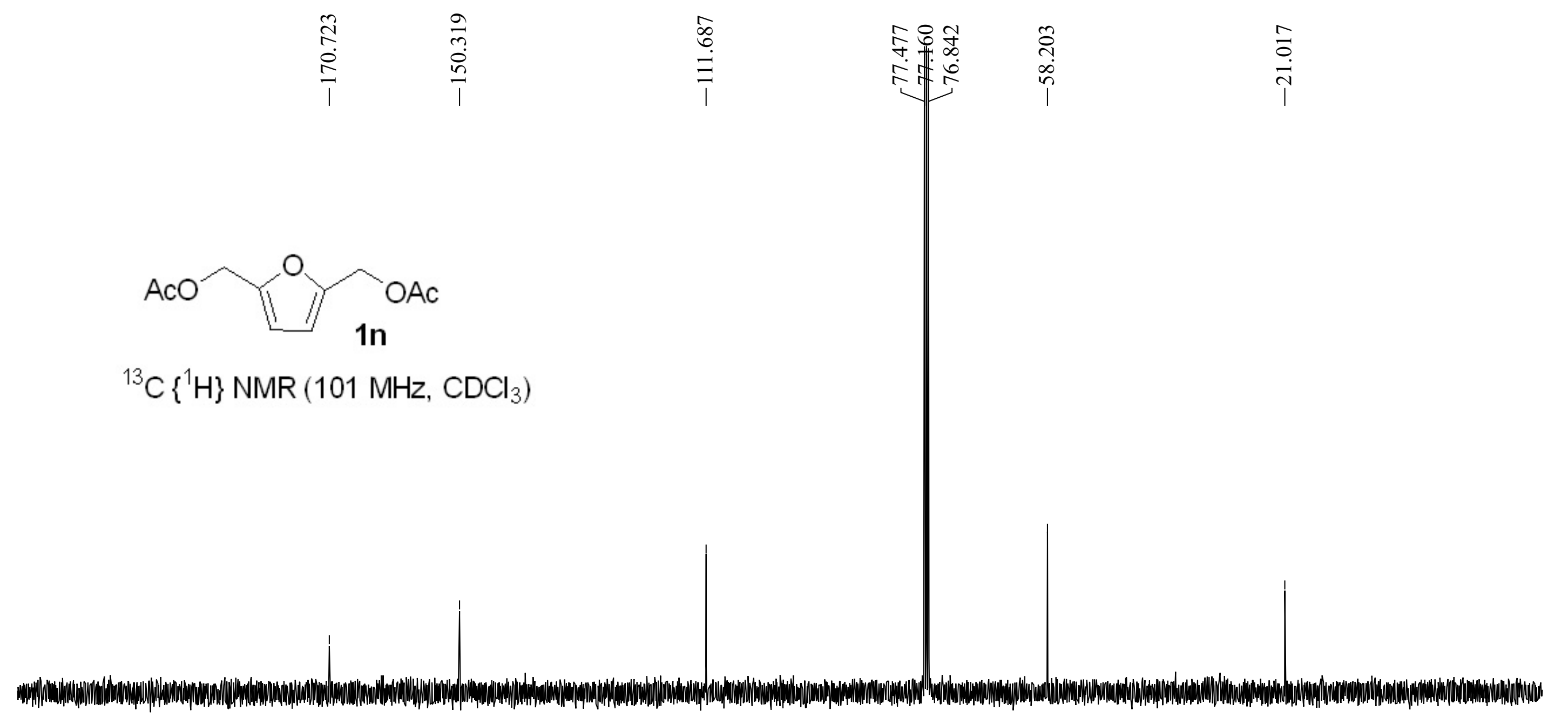

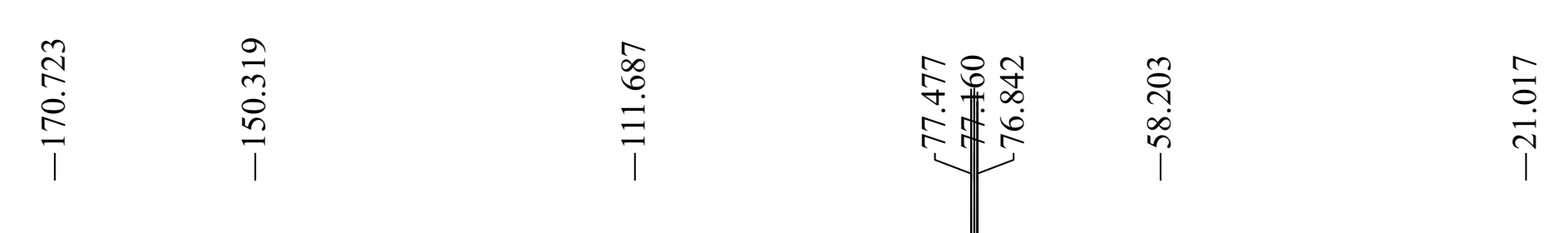

$\begin{array}{lllllllllllllllllllllllllll}210 & 200 & 190 & 180 & 170 & 160 & 150 & 140 & 130 & 120 & 110 & 100 & 90 & 80 & 70 & 60 & 50 & 40 & 30 & 20 & 10 & 0 & -10 \\ \text { f1 (ppm) }\end{array}$




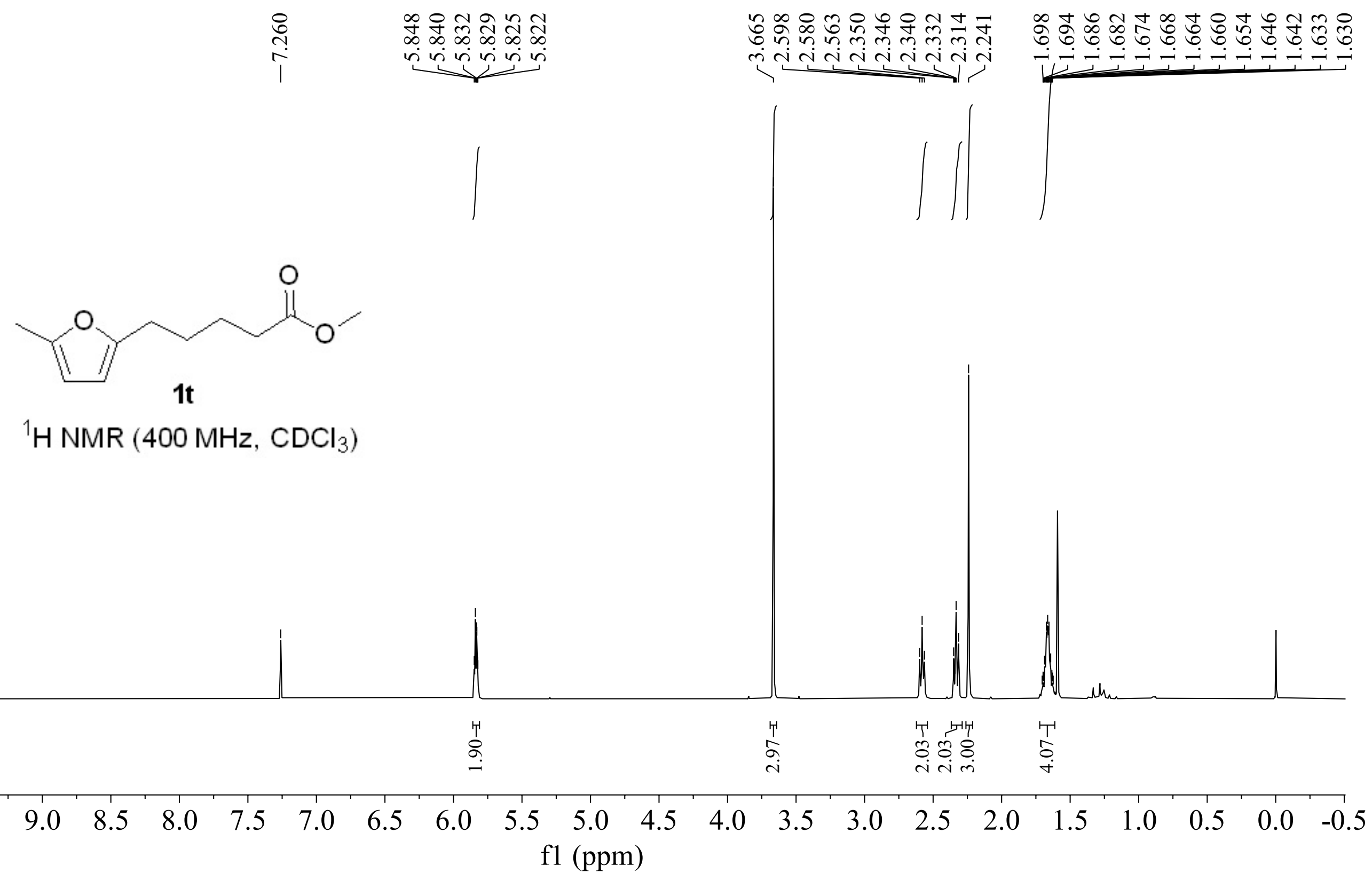




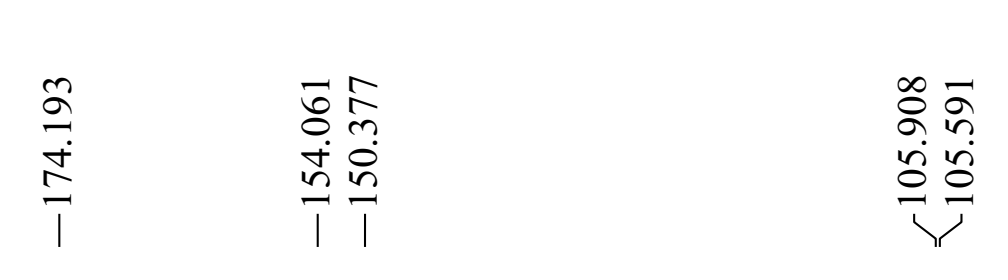

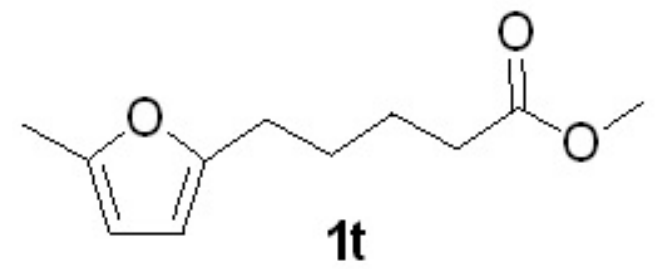

${ }^{13} \mathrm{C}\left\{{ }^{1} \mathrm{H}\right\} \mathrm{NMR}\left(101 \mathrm{MHz}, \mathrm{CDCl}_{3}\right)$

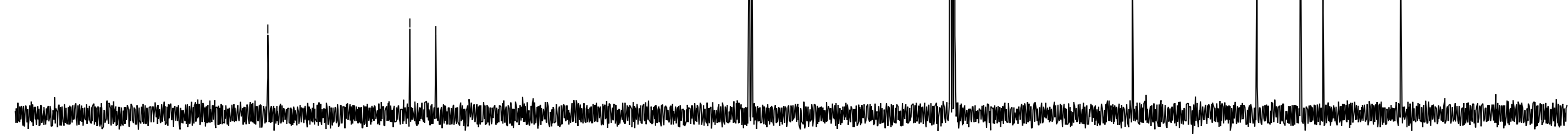

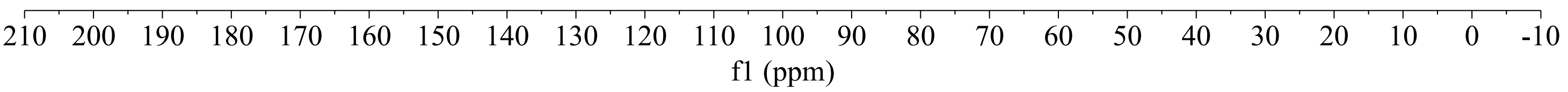




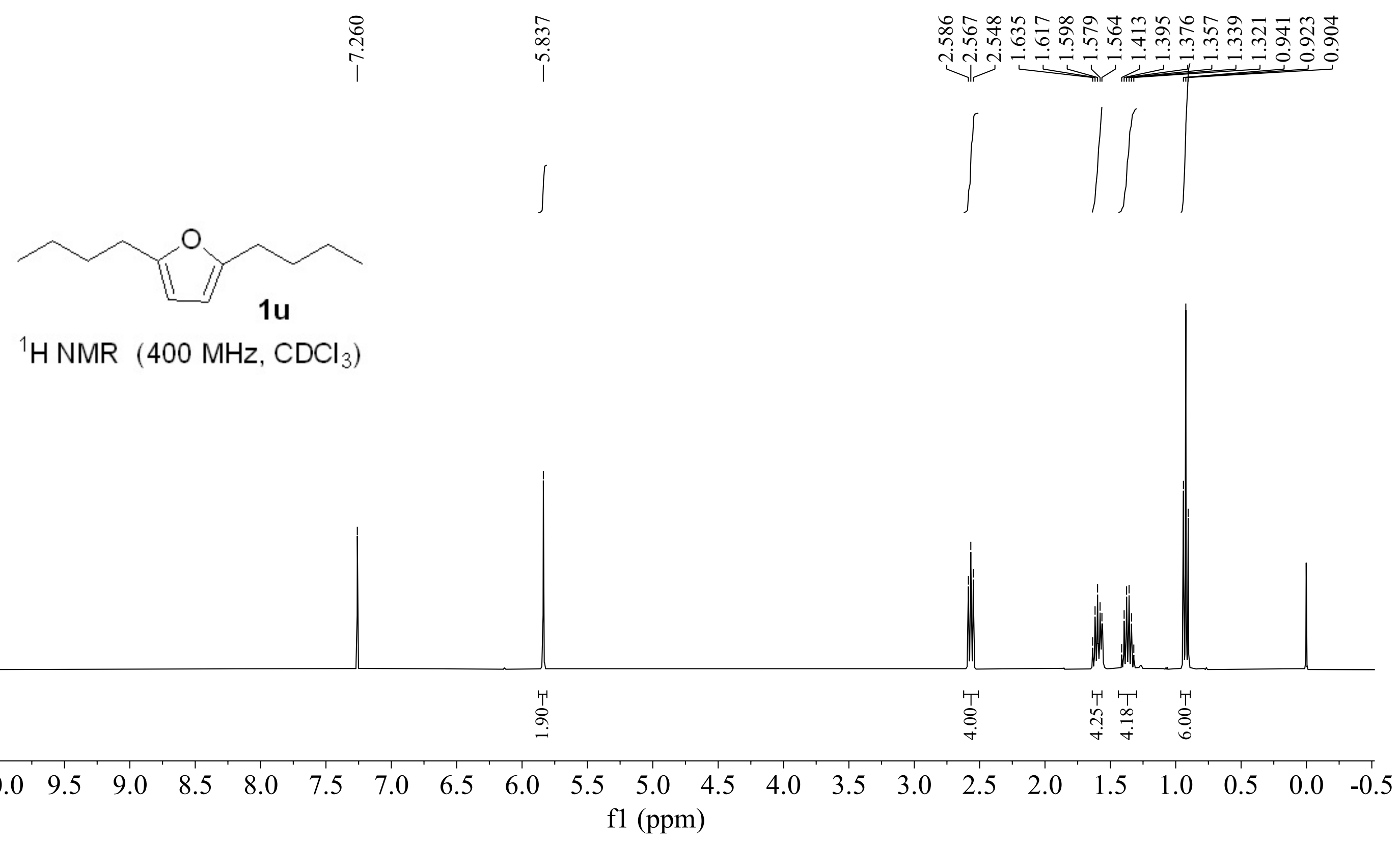




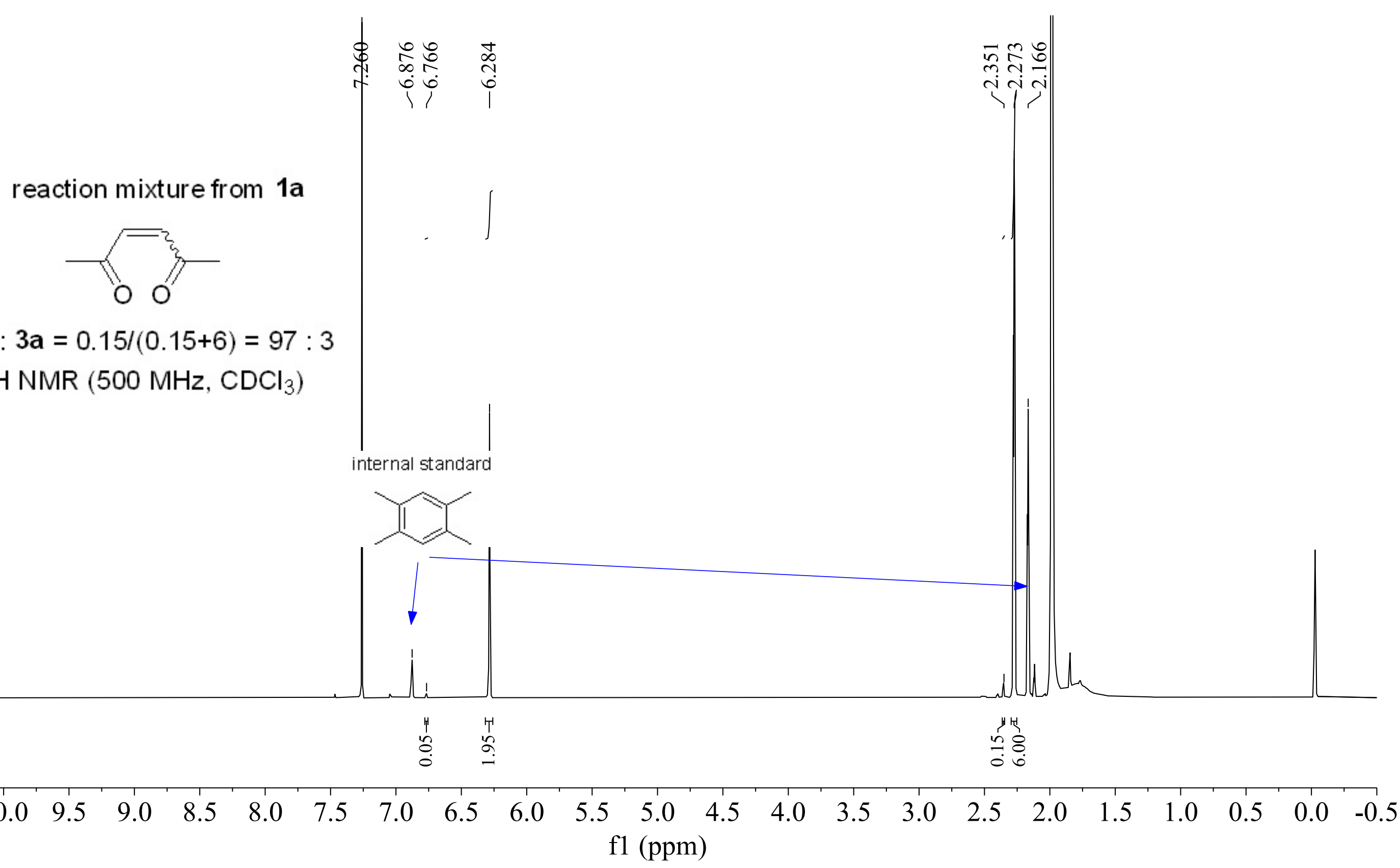




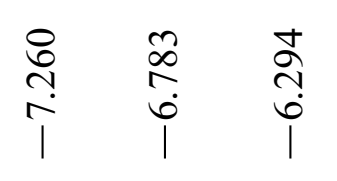

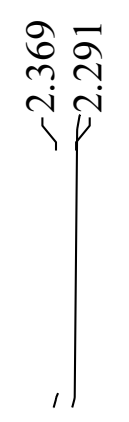<smiles>CC(=O)/C=C\C(=O)[CH+]C(C)=O</smiles>

$2 a: 3 a=95: 5$

${ }^{1} \mathrm{H} \operatorname{NMR}\left(400 \mathrm{MHz}, \mathrm{CDCl}_{3}\right)$

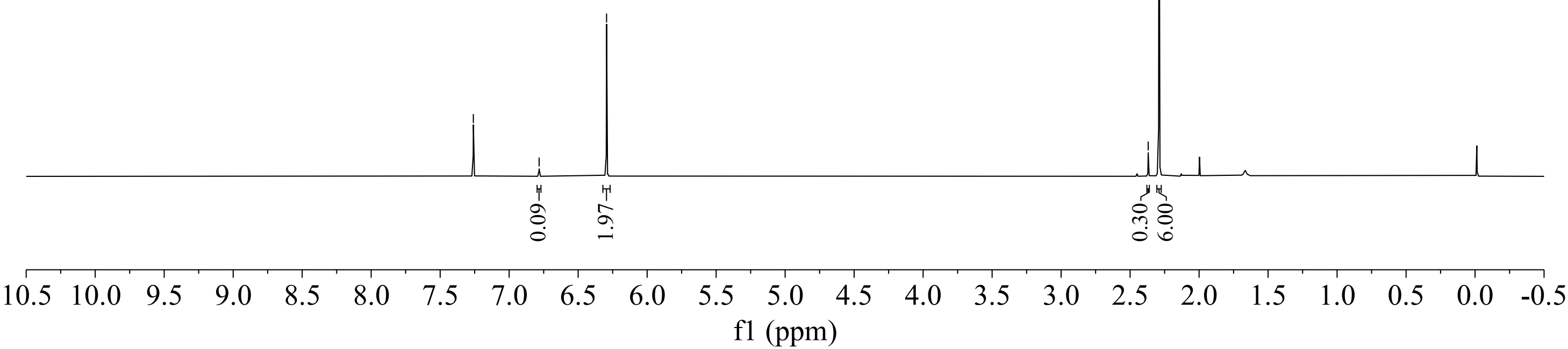

S37 


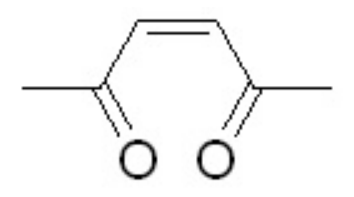

2a

${ }^{13} \mathrm{C}\left\{{ }^{1} \mathrm{H}\right\} \operatorname{NMR}\left(101 \mathrm{MHz}, \mathrm{CDCl}_{3}\right)$

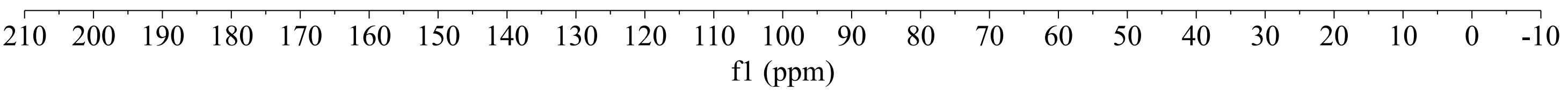


reaction mixture from $\mathbf{1 b}$<smiles>CC(=O)/C=C\C(=O)PC(C)=O</smiles>

2b $: \mathbf{3 b}=0.18 /(0.18+2)=92: 8$

${ }^{1} \mathrm{H} \mathrm{NMR}\left(500 \mathrm{MHz}, \mathrm{CDCl}_{3}\right)$

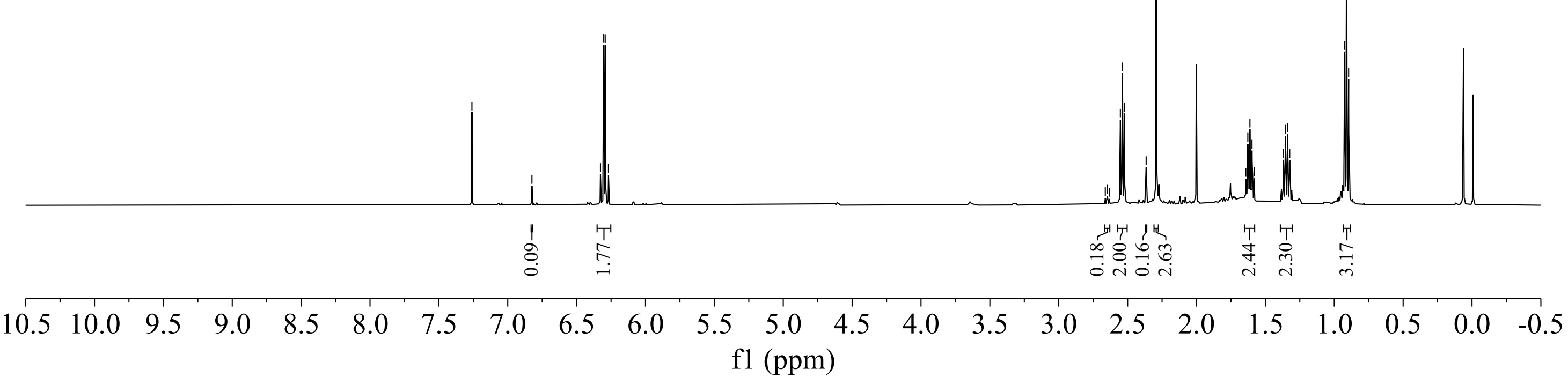




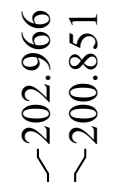

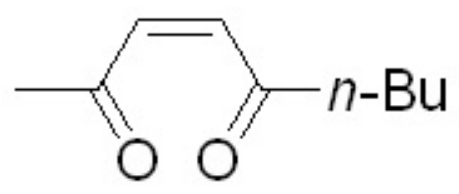

2b

${ }^{13} \mathrm{C}\left\{{ }^{1} \mathrm{H}\right\} \operatorname{NMR}\left(126 \mathrm{MHz}, \mathrm{CDCl}_{3}\right)$

$\begin{array}{rlllllllllllllllllllllllll}210 & 200 & 190 & 180 & 170 & 160 & 150 & 140 & 130 & 120 & 110 & 100 & 90 & 80 & 70 & 60 & 50 & 40 & 30 & 20 & 10 & 0 & -10 \\ \mathrm{fl}(\mathrm{ppm})\end{array}$



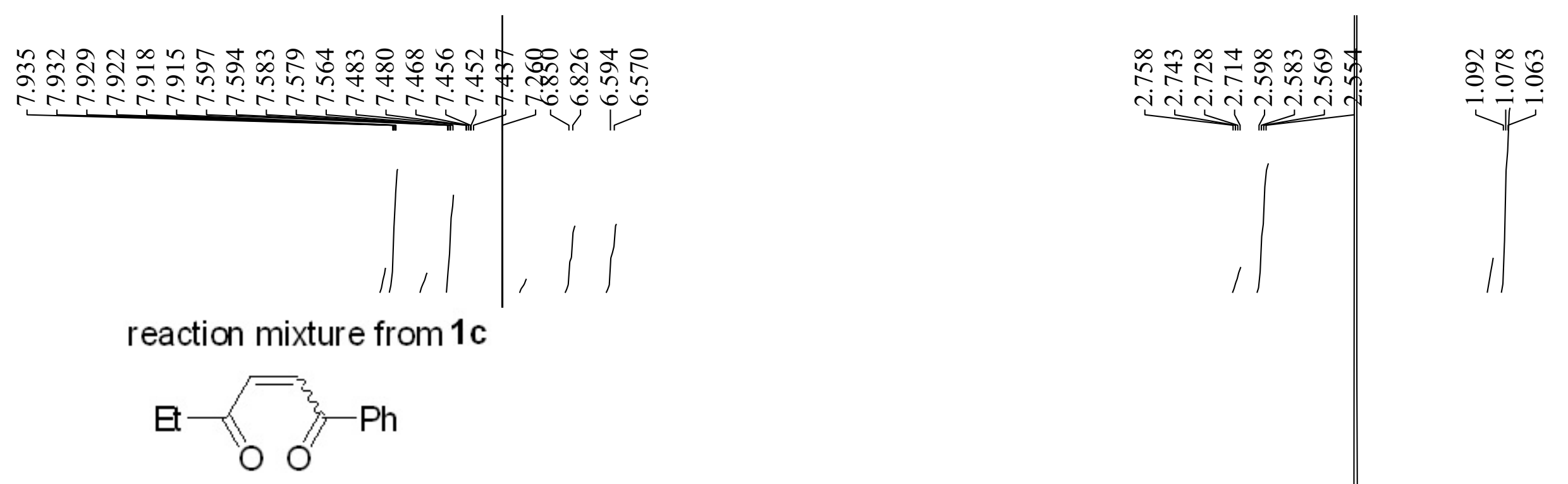

2c $: 3 c=0.4 /(0.4+2)=83: 17$

${ }^{1} \mathrm{H}$ NMR $\left(400 \mathrm{MHz}, \mathrm{CDCl}_{3}\right)$

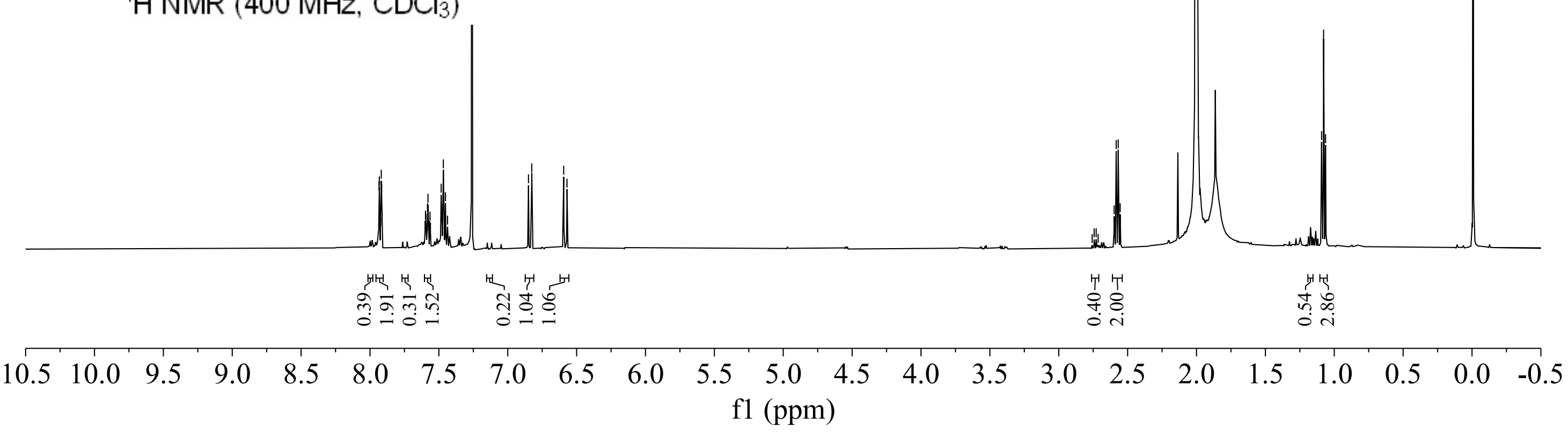




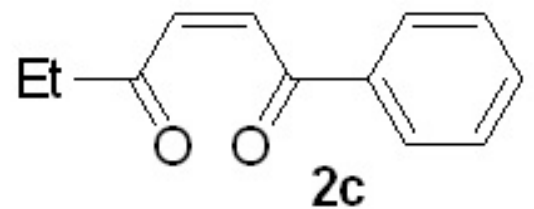

${ }^{1} \mathrm{H} \operatorname{NMR}\left(500 \mathrm{MHz}, \mathrm{CDCl}_{3}\right)$

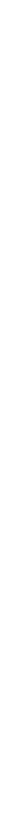




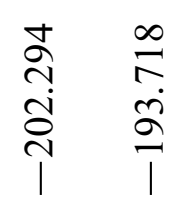

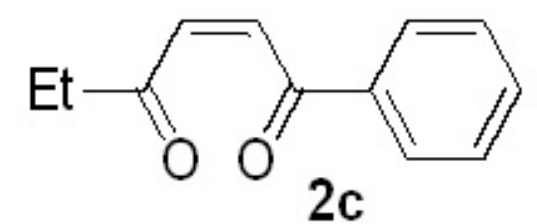

${ }^{13} \mathrm{C}\left\{{ }^{1} \mathrm{H}\right\} \operatorname{NMR}\left(\mathrm{CDCl}_{3}, 126 \mathrm{MHz}\right)$

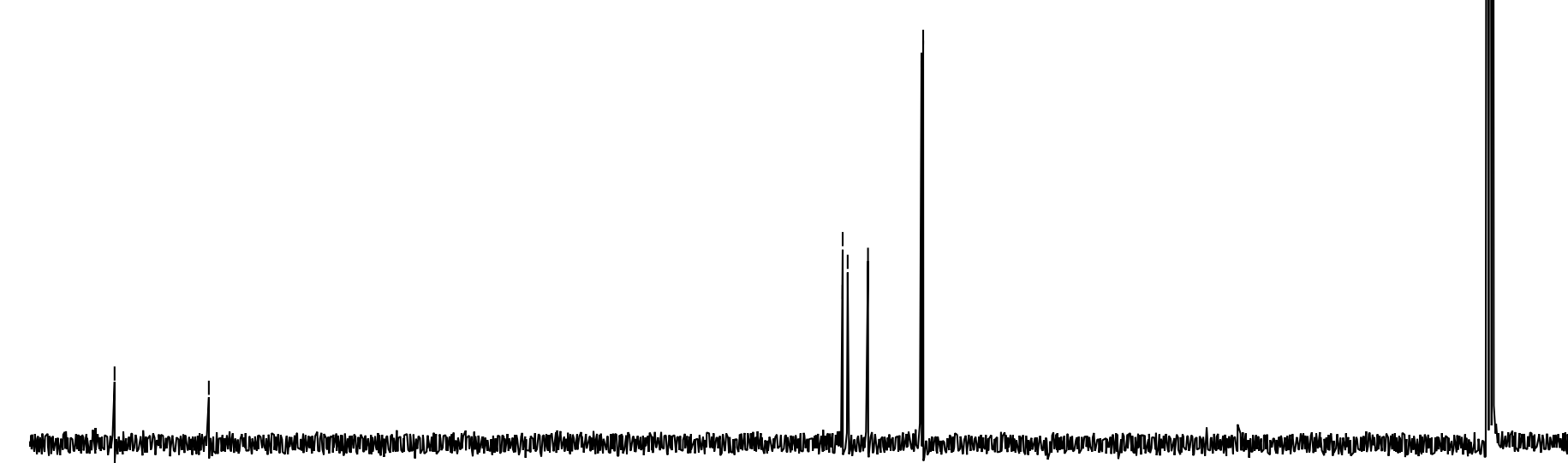

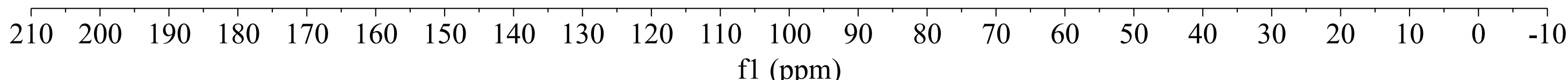




\begin{tabular}{|c|c|c|c|c|c|c|}
\hline$\frac{+}{\stackrel{+}{a}}$ & $\begin{array}{l}\hat{o} \\
\dot{0} \\
\hat{0}\end{array}$ & 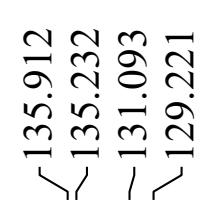 & $\begin{array}{l}\stackrel{2}{ \pm} \\
\stackrel{+}{ \pm} \\
1\end{array}$ & 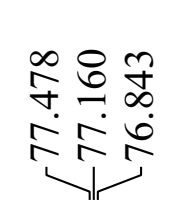 & 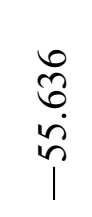 & $\begin{array}{l}\text { के } \\
\dot{0} \\
\hat{0}\end{array}$ \\
\hline
\end{tabular}<smiles>CCC(=O)/C=C\C(=O)c1ccc(OC)cc1</smiles>

${ }^{13} \mathrm{C}\left\{{ }^{1} \mathrm{H}\right\}$ NMR $\left(101 \mathrm{MHz}, \mathrm{CDCl}_{3}\right)$

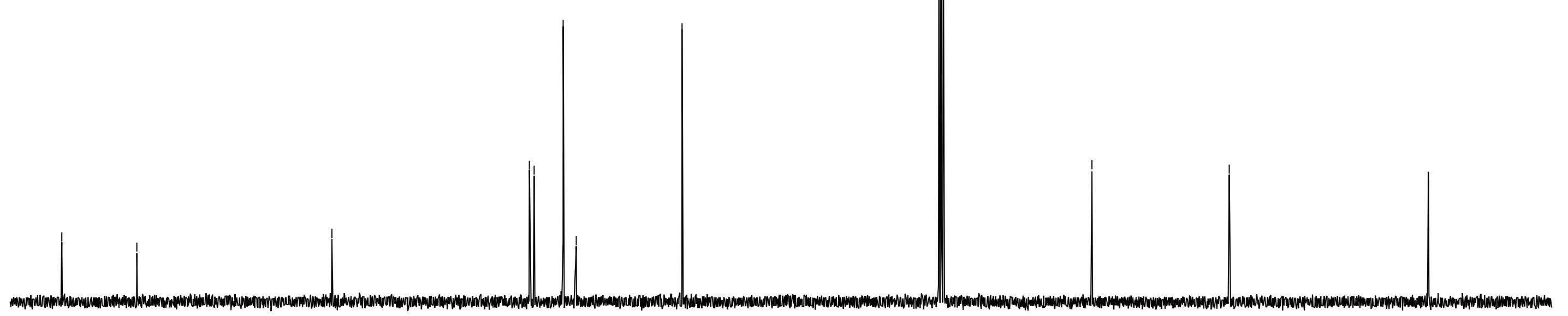

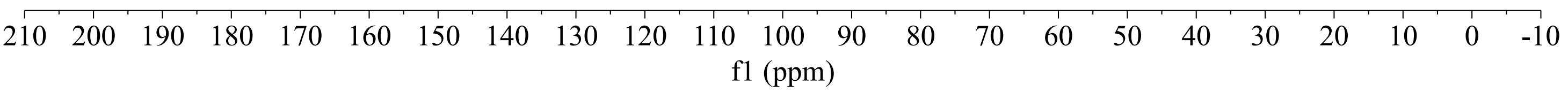




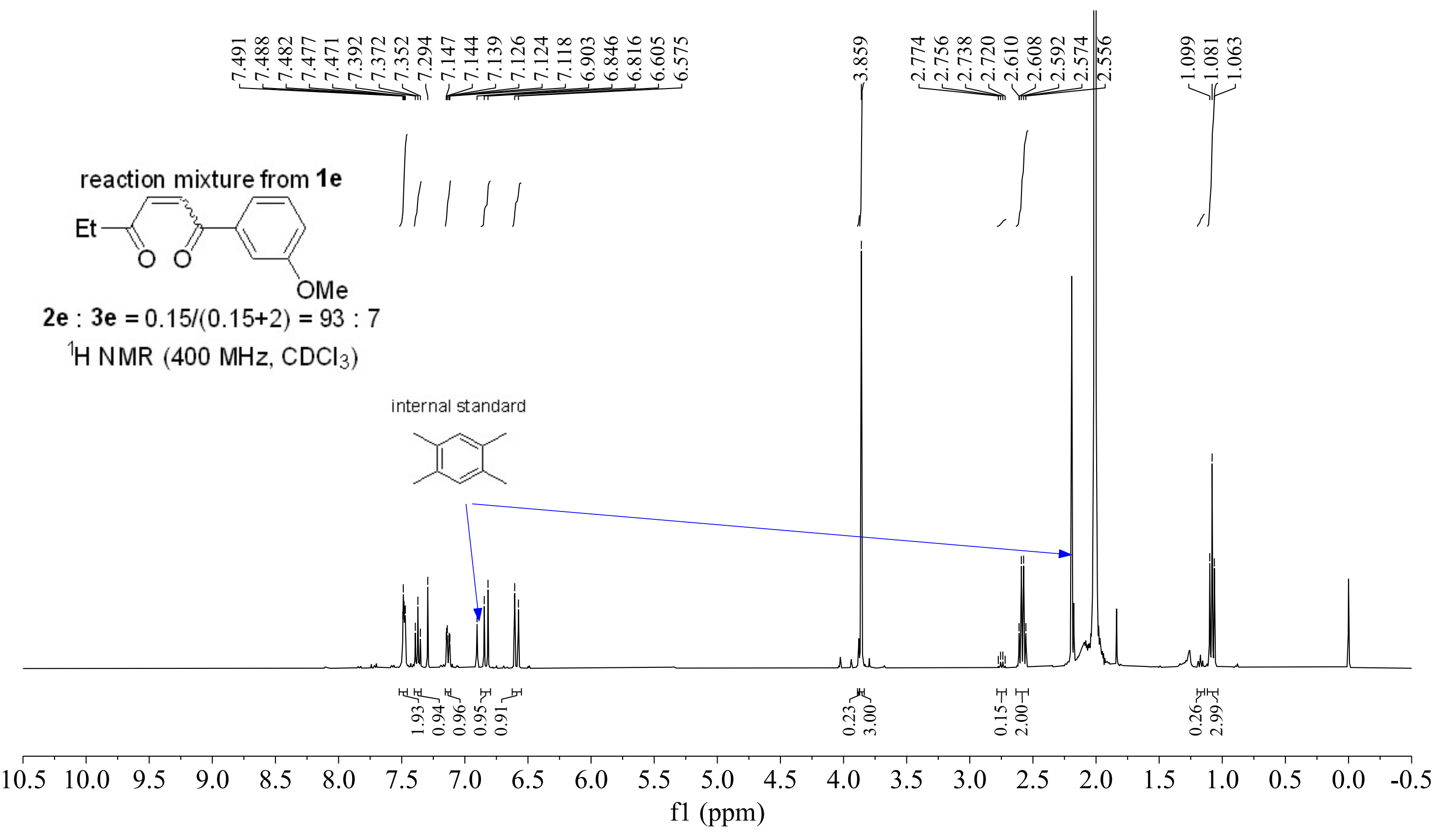




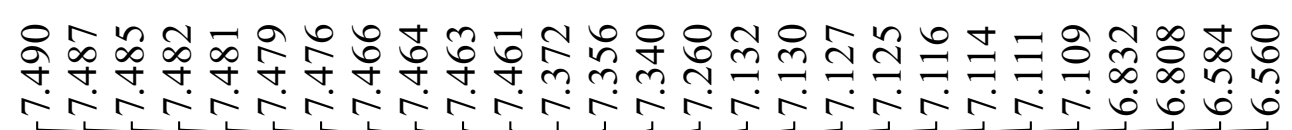
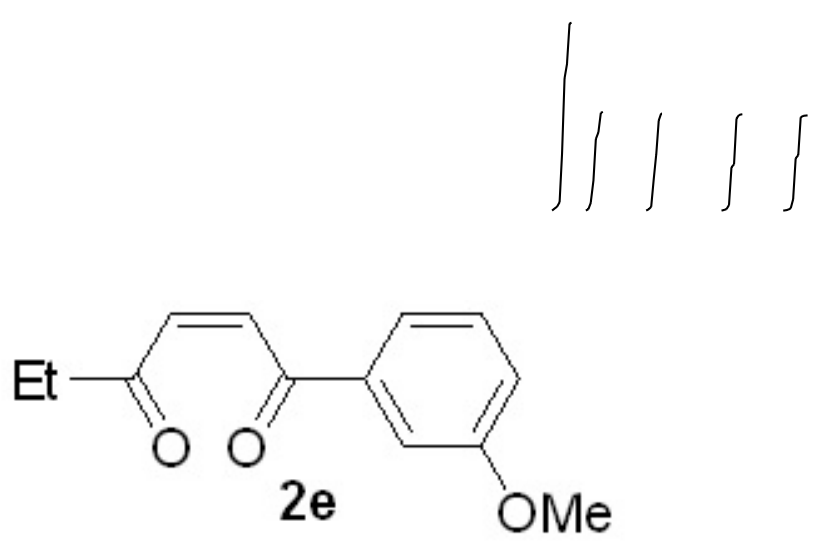

${ }^{1} \mathrm{H}$ NMR $\left(500 \mathrm{MHz}, \mathrm{CDCl}_{3}\right)$

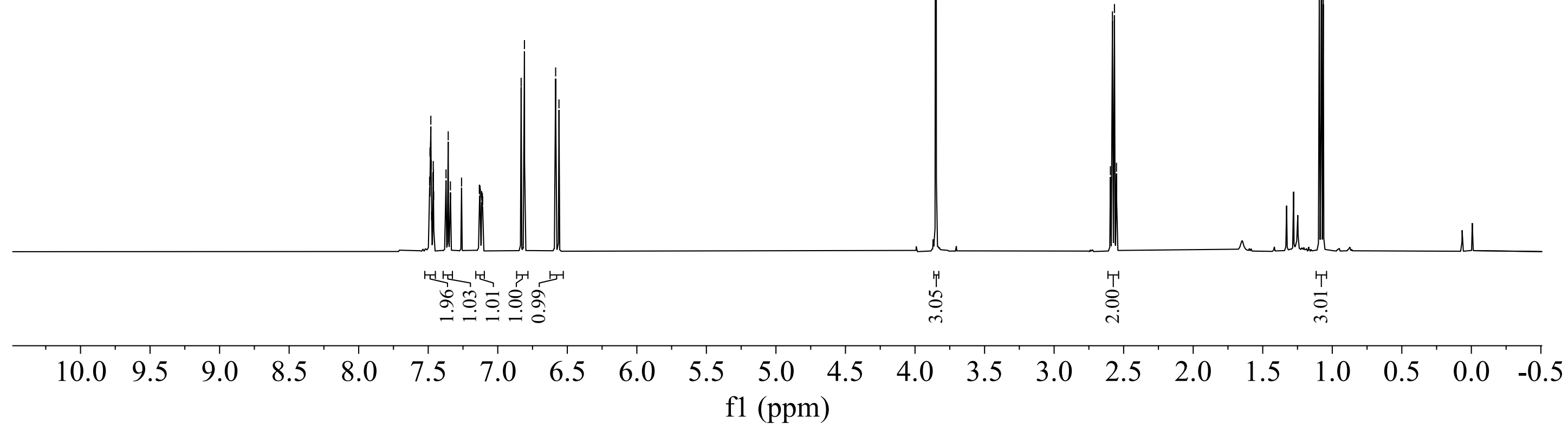




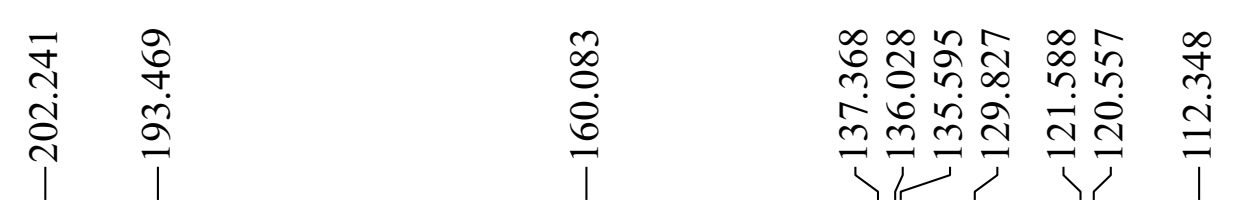

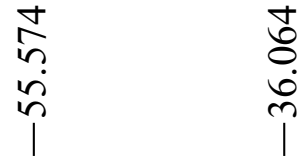

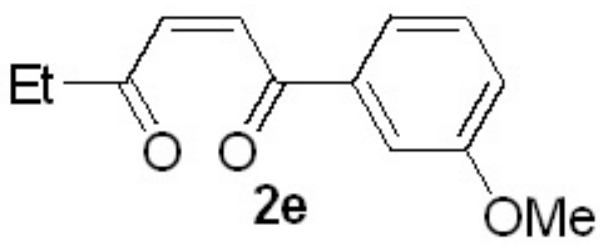

${ }^{13} \mathrm{C}\left\{{ }^{1} \mathrm{H}\right\} \operatorname{NMR}\left(126 \mathrm{MHz}, \mathrm{CDCl}_{3}\right)$

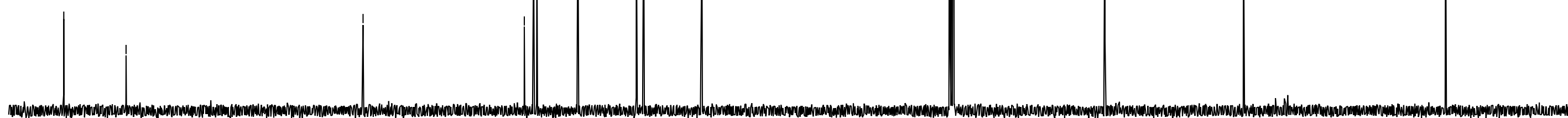

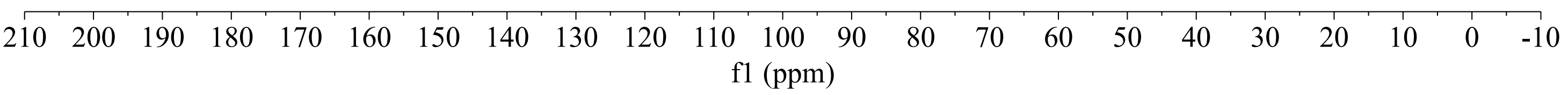



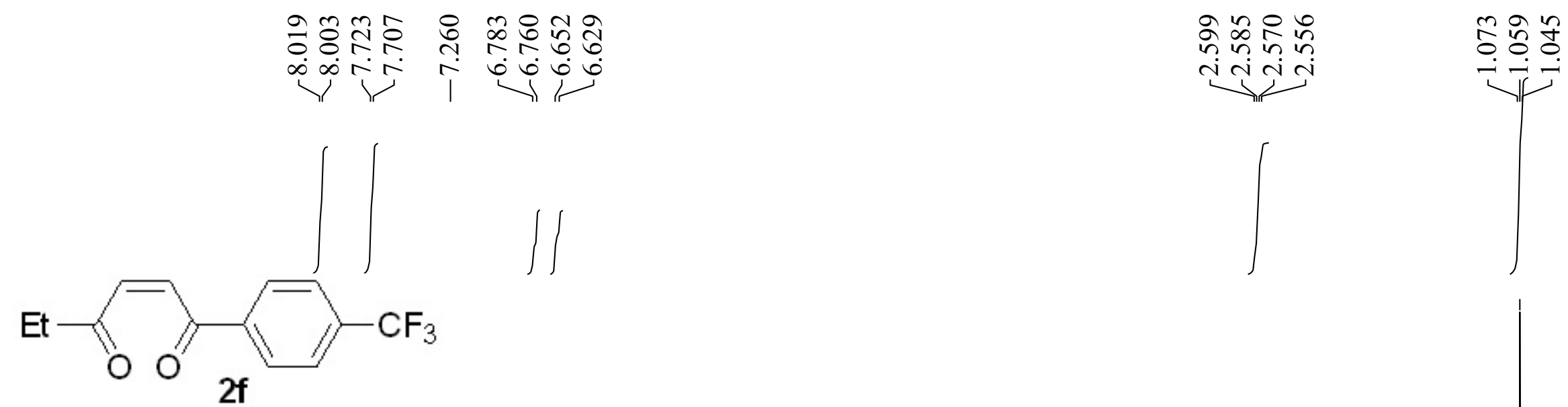

${ }^{1} \mathrm{H}$ NMR $\left(500 \mathrm{MHz}, \mathrm{CDCl}_{3}\right)$

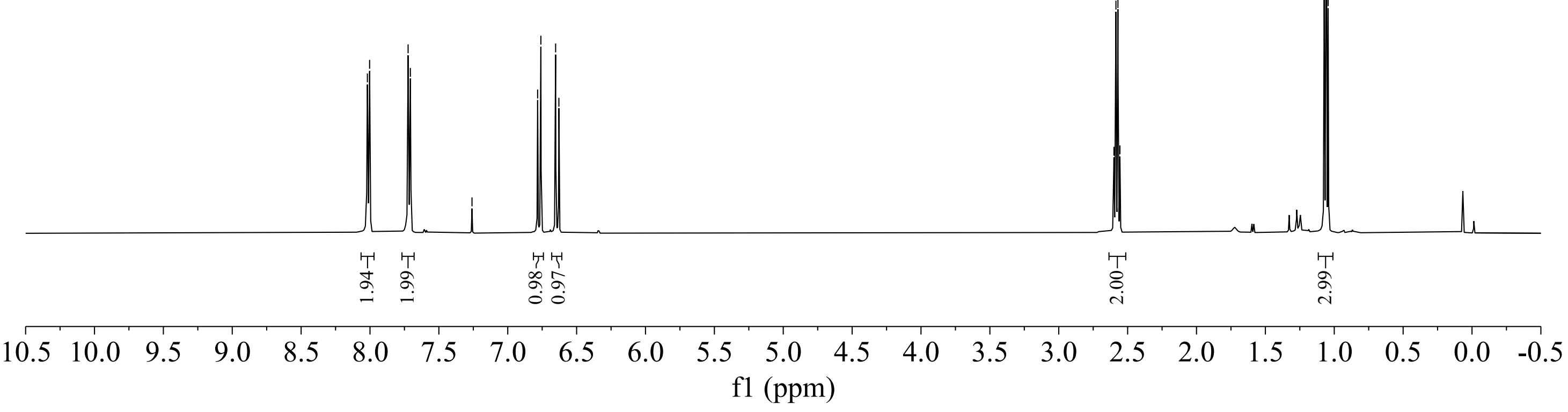




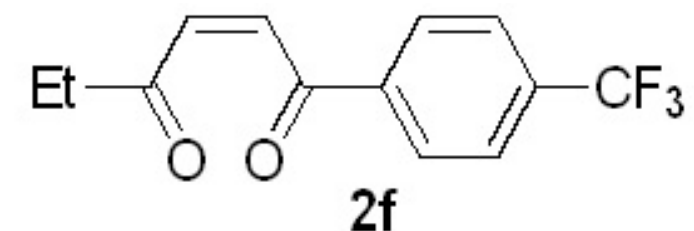

${ }^{13} \mathrm{C}\left\{{ }^{1} \mathrm{H}\right\}$ NMR $\left(126 \mathrm{MHz}, \mathrm{CDCl}_{3}\right)$

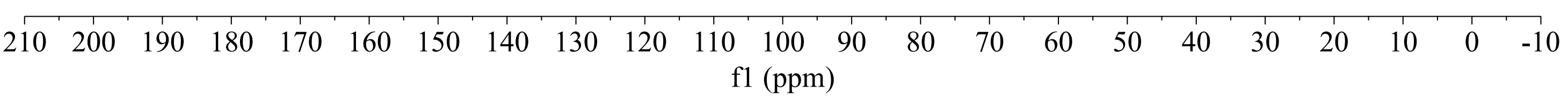




\section{$\frac{6}{6}$}

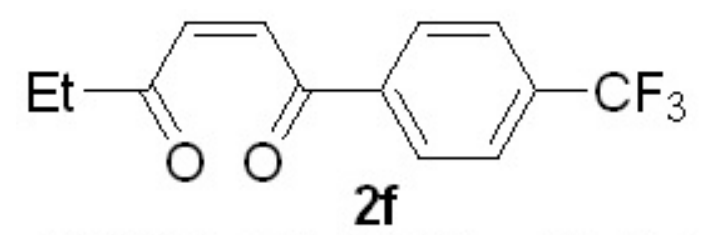

${ }^{19} \mathrm{~F} \mathrm{NMR}\left(371 \mathrm{MHz}, \mathrm{CDCl}_{3}\right)$

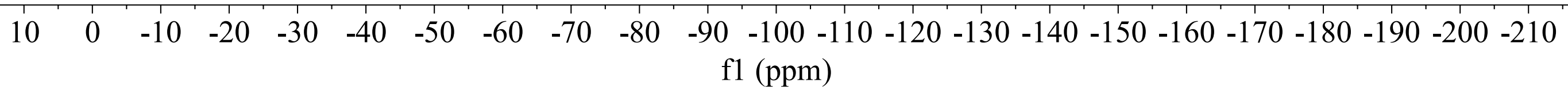


reaction mixture from $\mathbf{1 g}$
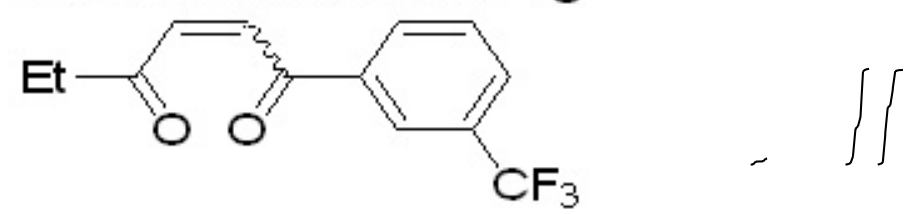

$\mathbf{2 g}: \mathbf{3 g}=0.16 /(0.16+2)=93: 7$

${ }^{1} \mathrm{H}$ NMR $\left(400 \mathrm{MHz}, \mathrm{CDCl}_{3}\right)$

internal standard
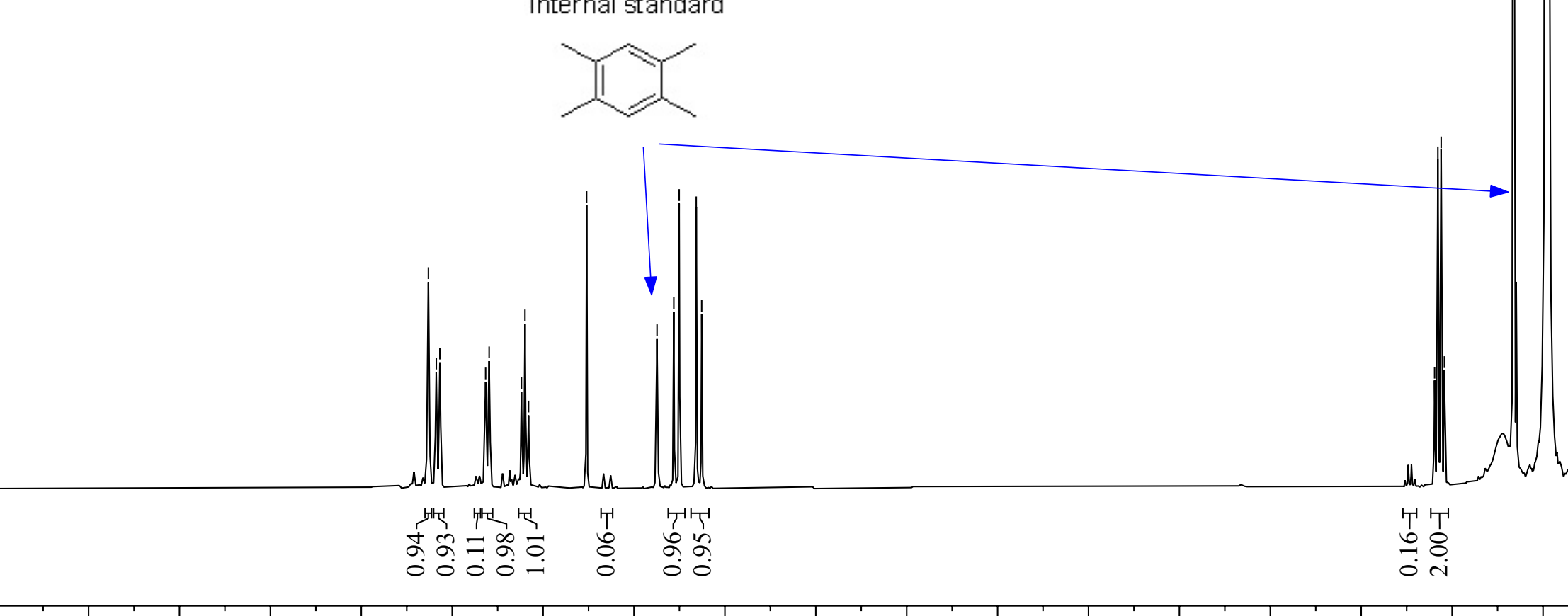

$\begin{array}{llllllllllllllllllllllll}10.5 & 10.0 & 9.5 & 9.0 & 8.5 & 8.0 & 7.5 & 7.0 & 6.5 & 6.0 & 5.5 & 5.0 & 4.5 & 4.0 & 3.5 & 3.0 & 2.5 & 2.0 & 1.5 & 1.0 & 0.5 & 0.0 & -0.5 \\ & & & & & & & \end{array}$ 


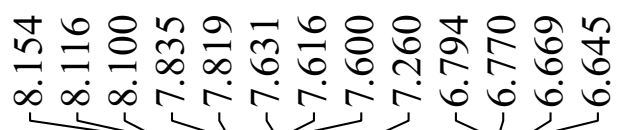
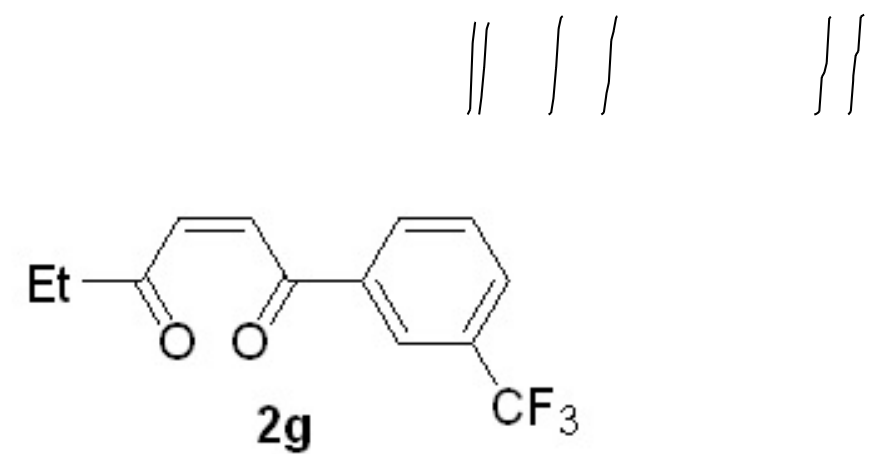

${ }^{1} \mathrm{H}$ NMR $\left(500 \mathrm{MHz}, \mathrm{CDCl}_{3}\right)$

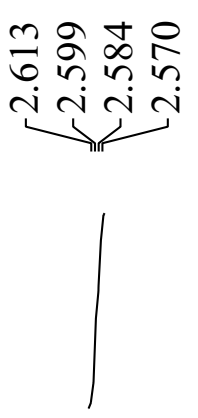

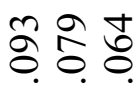
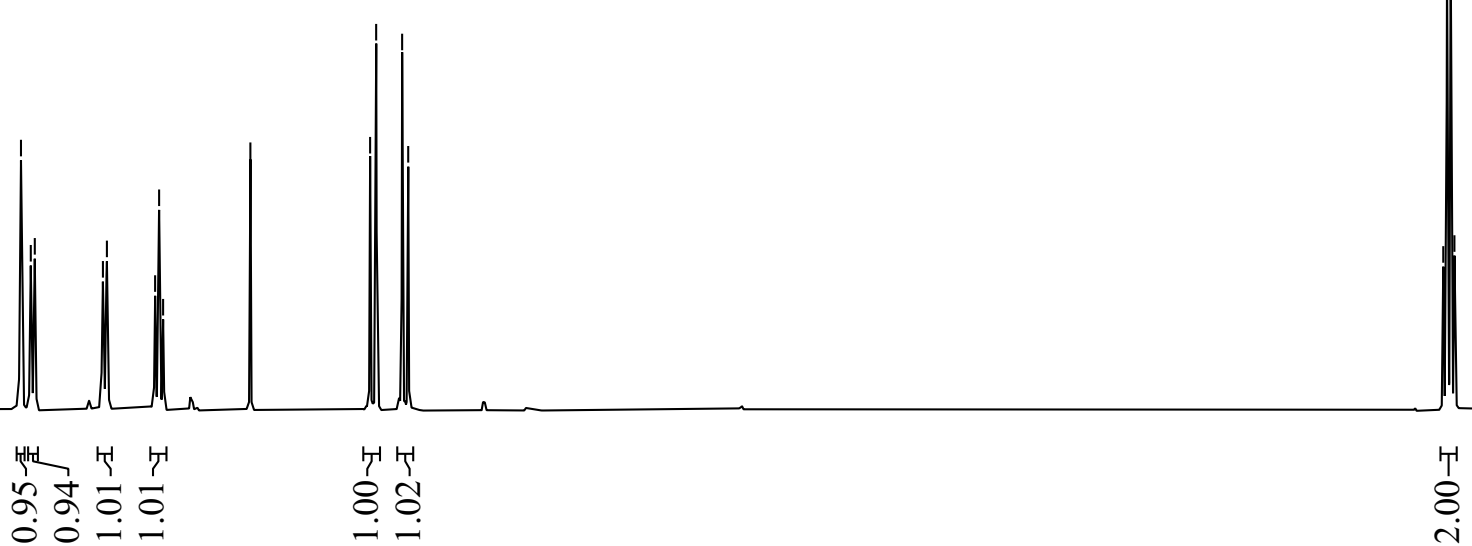

T'
$\stackrel{8}{8}$
$\dot{m}$

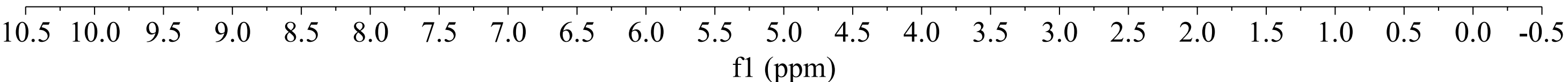




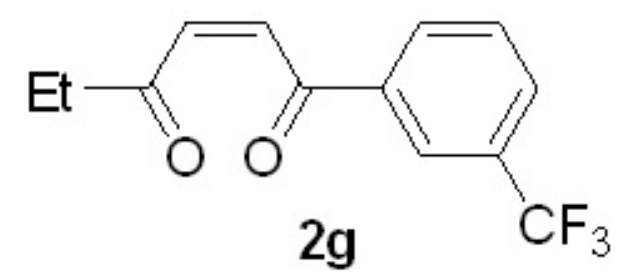

${ }^{19} \mathrm{~F}$ NMR (471 MHz, $\mathrm{CDCl}_{3}$ )

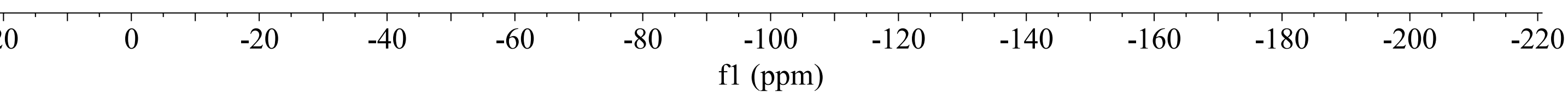




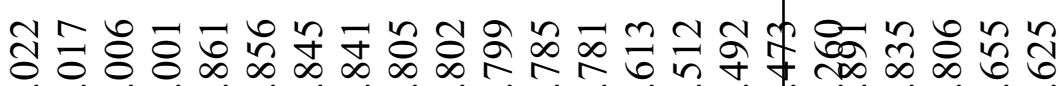

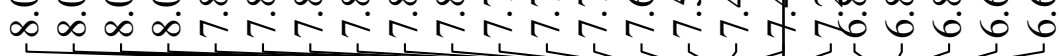

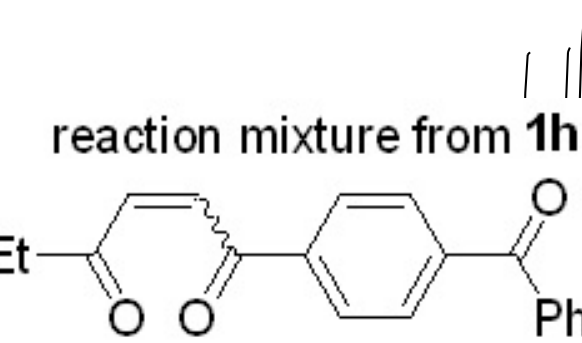

\section{II}

h

$\mathbf{2 h}: \mathbf{3 h}=0.25 /(0.25+2)=89: 11$

${ }^{1} \mathrm{H}$ NMR $\left(400 \mathrm{MHz}, \mathrm{CDCl}_{3}\right)$

react $22 \mathrm{~h}$
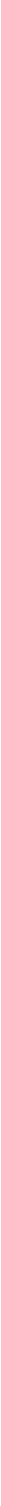


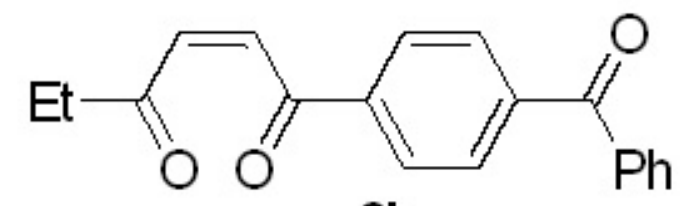
2h

${ }^{13} \mathrm{C}\left\{{ }^{1} \mathrm{H}\right\} \mathrm{NMR}\left(101 \mathrm{MHz}, \mathrm{CDCl}_{3}\right)$

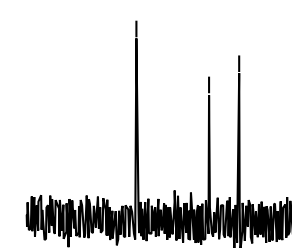

\begin{tabular}{rlllllllllllllllllllllll}
\hline 10 & 200 & 190 & 180 & 170 & 160 & 150 & 140 & 130 & 120 & 110 & 100 & 90 & 80 & 70 & 60 & 50 & 40 & 30 & 20 & 10 & 0 & \\
f1 (ppm)
\end{tabular}


reaction mixture from $\mathbf{1 i}$
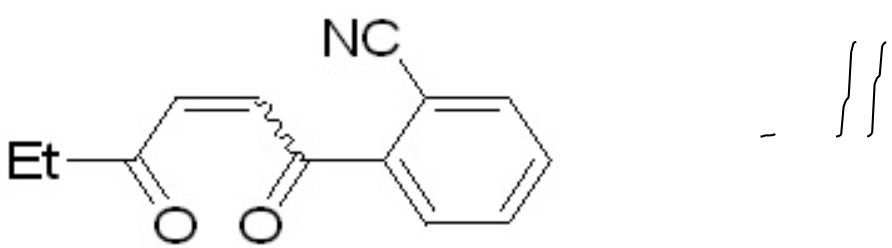

$2 \mathbf{i}: 3 \mathbf{i}=0.1 /(0.1+2)=95: 5$

${ }^{1} \mathrm{H}$ NMR $\left(400 \mathrm{MHz}, \mathrm{CDCl}_{3}\right)$ internal standard

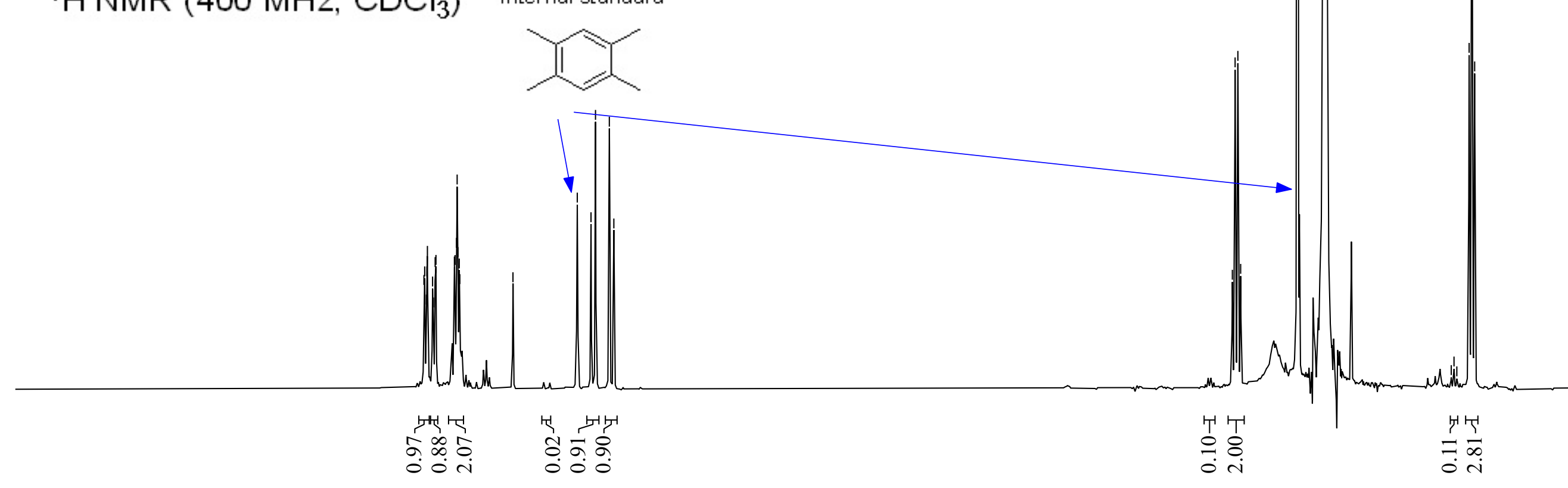

$\begin{array}{llll}1.0 & 0.5 & 0.0 & -0.5\end{array}$ $\begin{array}{lllllllllll}10.0 & 9.5 & 9.0 & 8.5 & 8.0 & 7.5 & 7.0 & 6.5 & 6.0 & 5.5 & \begin{array}{c}5.0 \\ \mathrm{f} 1\end{array}(\mathrm{ppm})\end{array}$ 


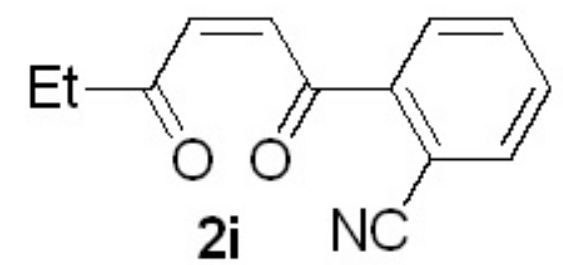

${ }^{1} \mathrm{HNMR}\left(400 \mathrm{MHz}, \mathrm{CDCl}_{3}\right)$

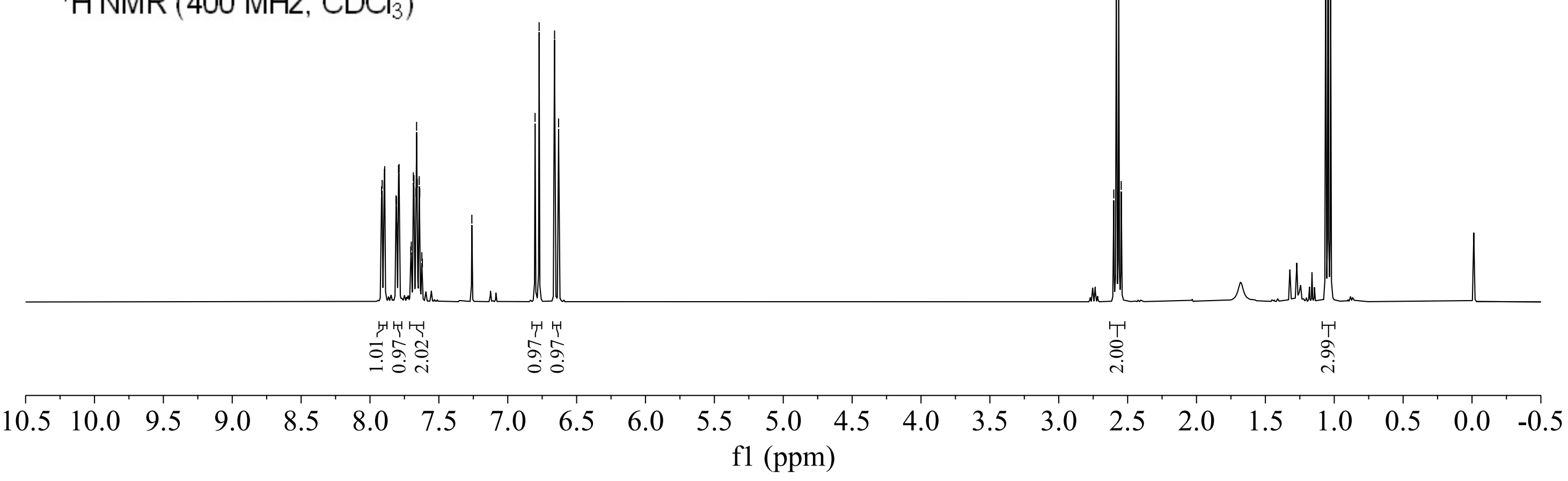




$$
\text { 产章 }
$$

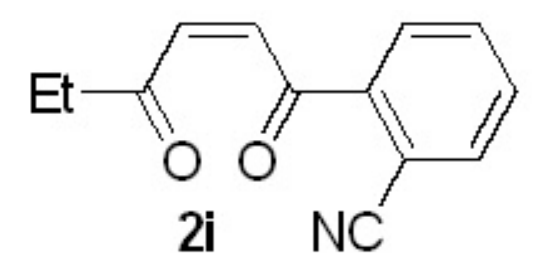

${ }^{13} \mathrm{C}\left\{{ }^{1} \mathrm{H}\right\} \mathrm{NMR}\left(101 \mathrm{MHz}, \mathrm{CDCl}_{3}\right)$

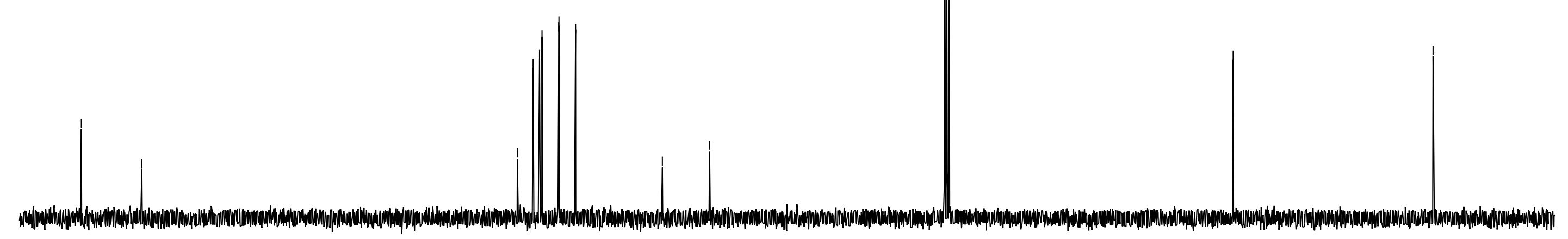

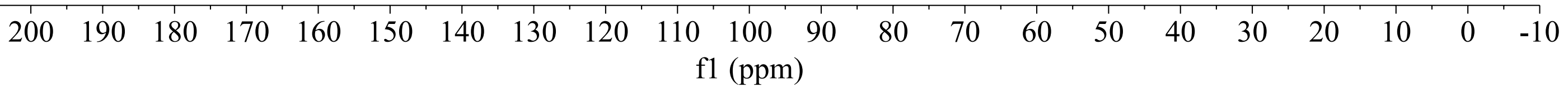




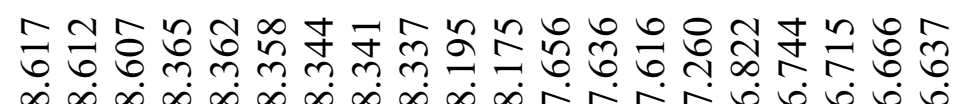

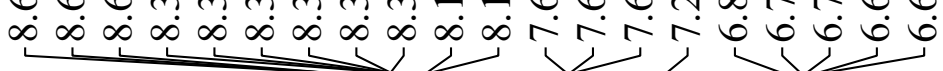

reaction mixture from $\mathbf{1 j}$

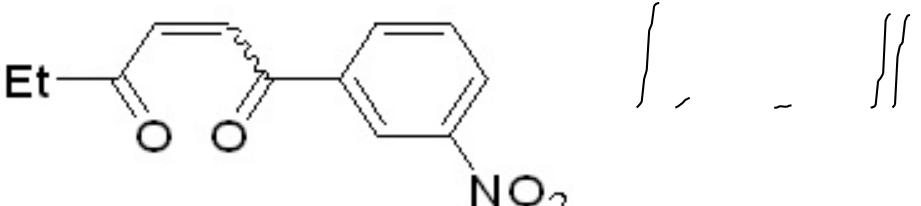

$\mathrm{NO}_{2}$

2j : $\mathbf{3 j}=0.13 /(0.13+2)=94: 6$

${ }^{1} \mathrm{H}$ NMR $\left(400 \mathrm{MHz}, \mathrm{CDCl}_{3}\right) \quad$ internal standard

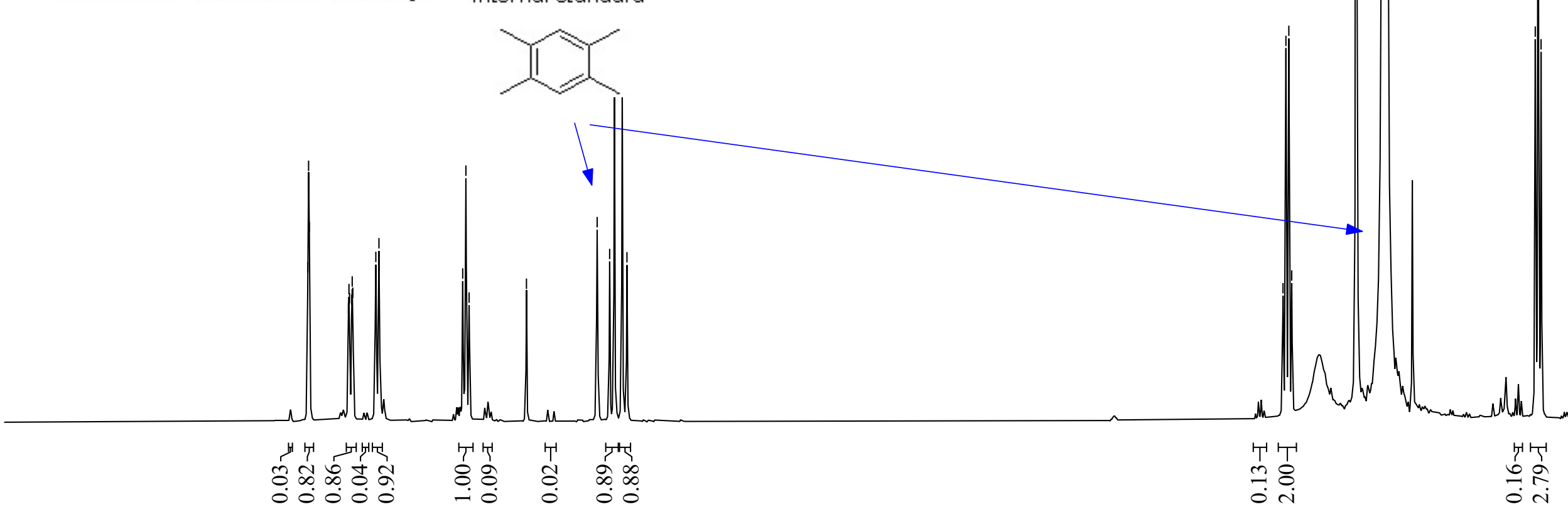

$$
\begin{array}{llllllllllllllllllllll}
10.0 & 9.5 & 9.0 & 8.5 & 8.0 & 7.5 & 7.0 & 6.5 & 6.0 & 5.5 & \begin{array}{c}
5.0 \\
\mathrm{f} 1(\mathrm{ppm})
\end{array} & 4.5 & 4.0 & 3.5 & 3.0 & 2.5 & 2.0 & 1.5 & 1.0 & 0.5 & 0.0 & -0.5
\end{array}
$$



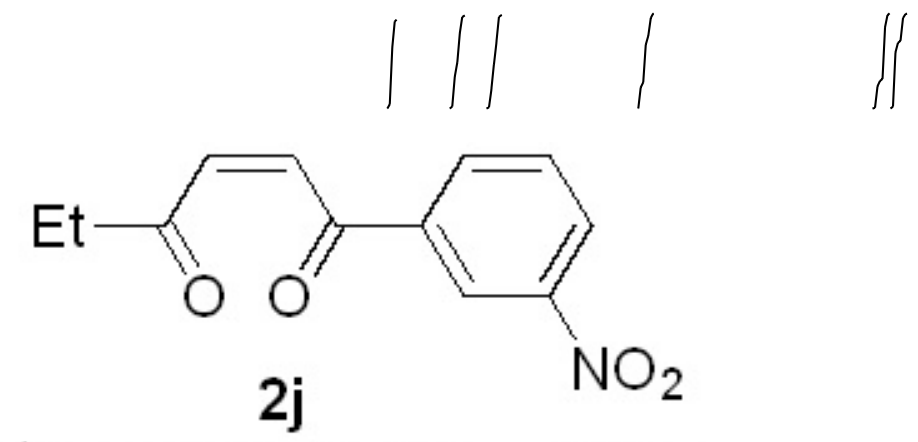

${ }^{1} \mathrm{H} \mathrm{NMR}\left(400 \mathrm{MHz}, \mathrm{CDCl}_{3}\right)$

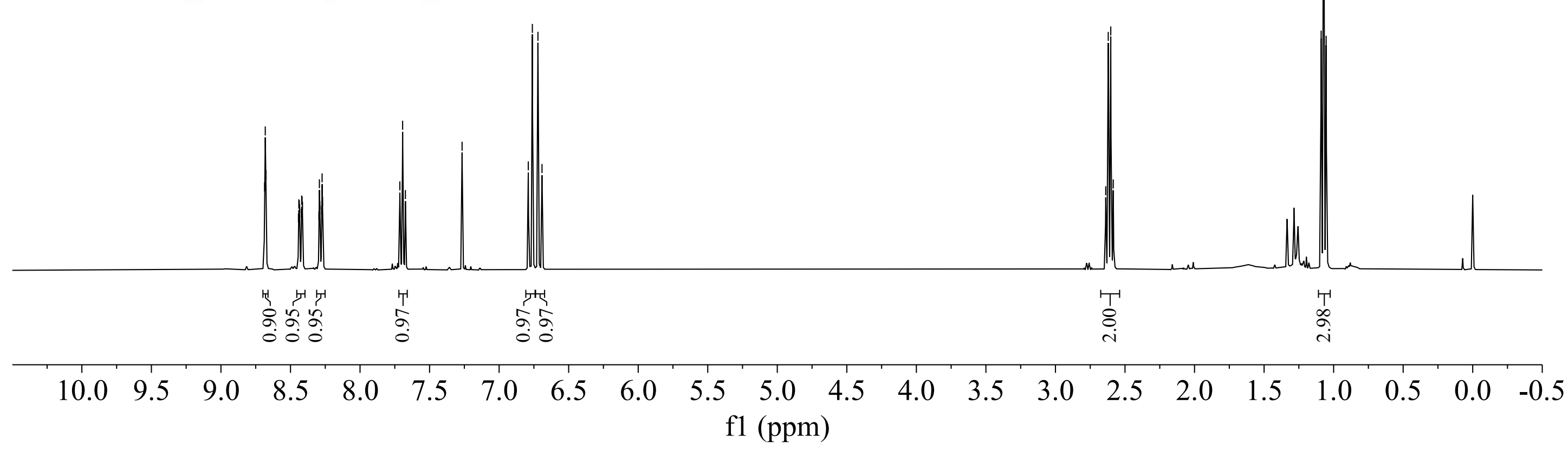




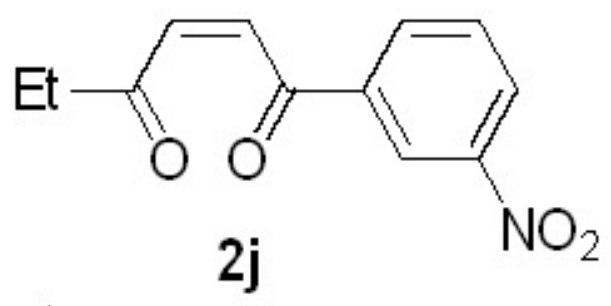

${ }^{13} \mathrm{C}\left\{{ }^{1} \mathrm{H}\right\} \operatorname{NMR}\left(101 \mathrm{MHz}, \mathrm{CDCl}_{3}\right)$
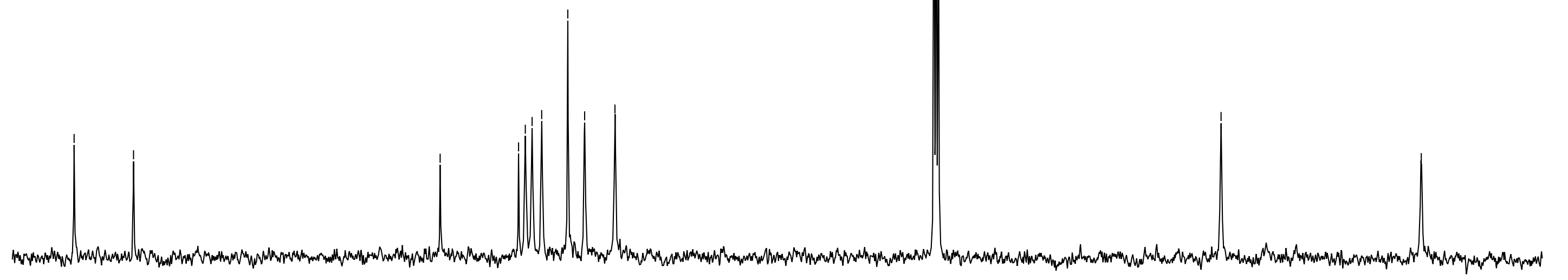

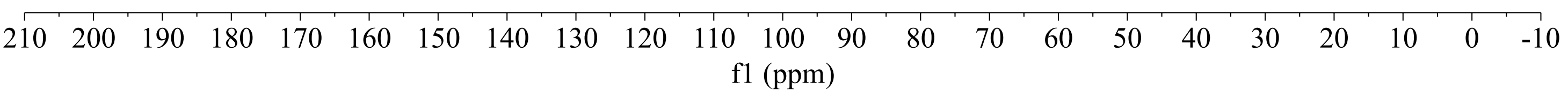




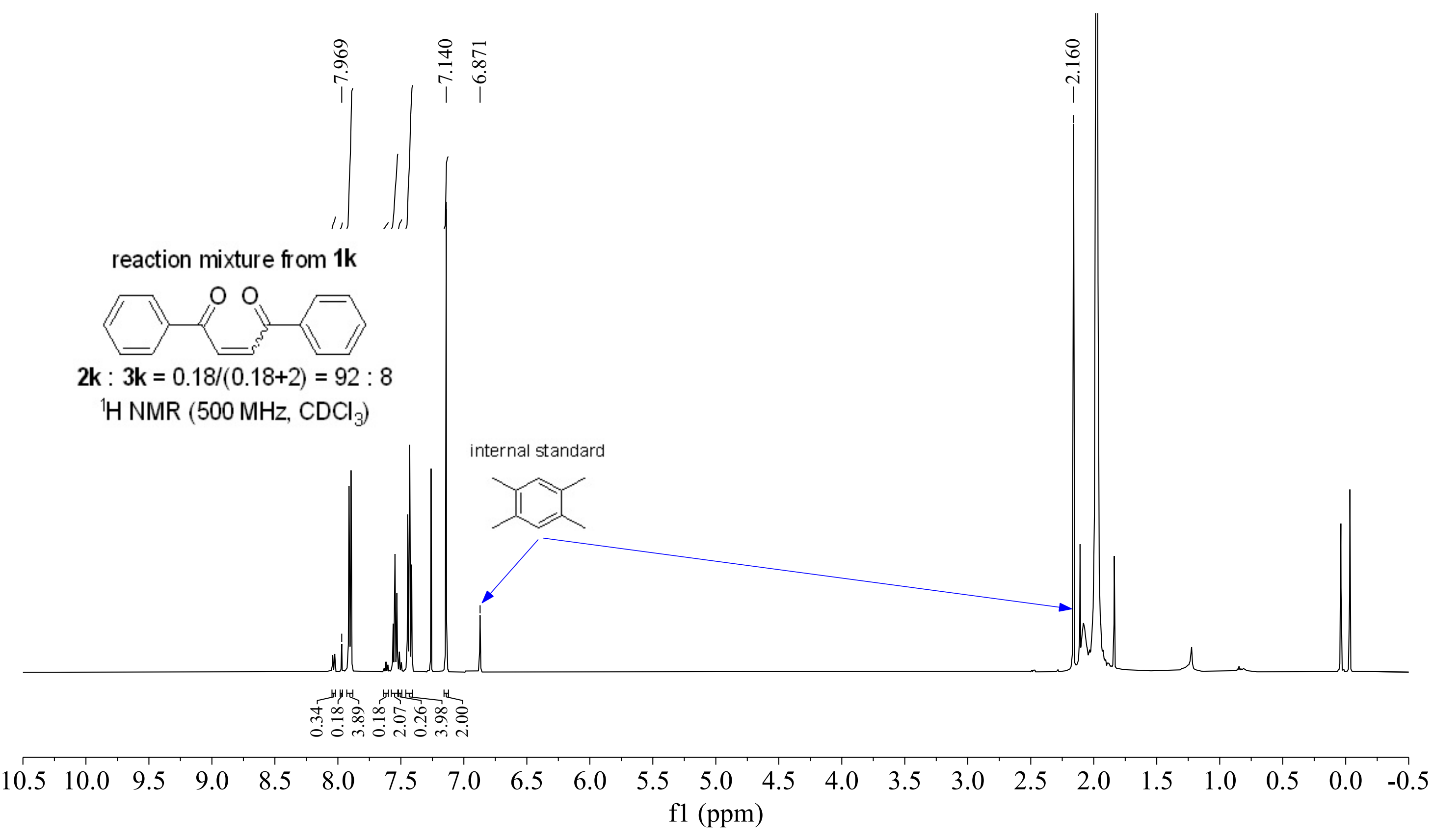



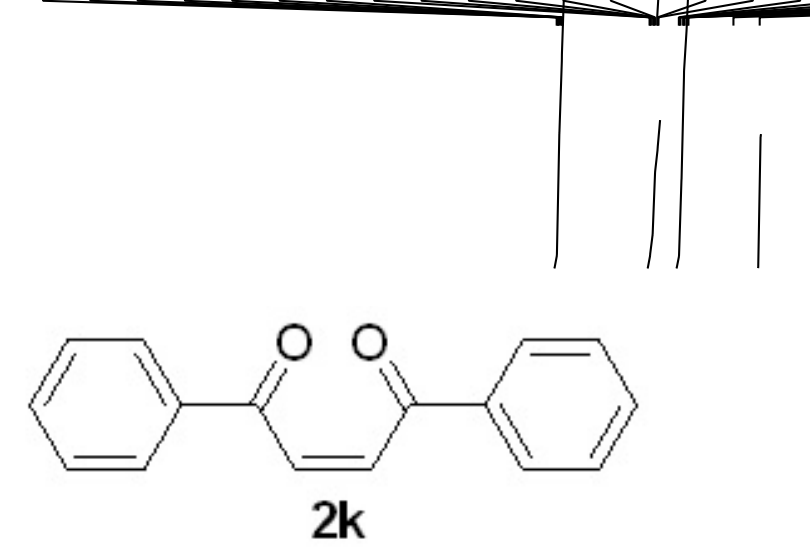

${ }^{1} \mathrm{H}$ NMR $\left(500 \mathrm{MHz}, \mathrm{CDCl}_{3}\right)$

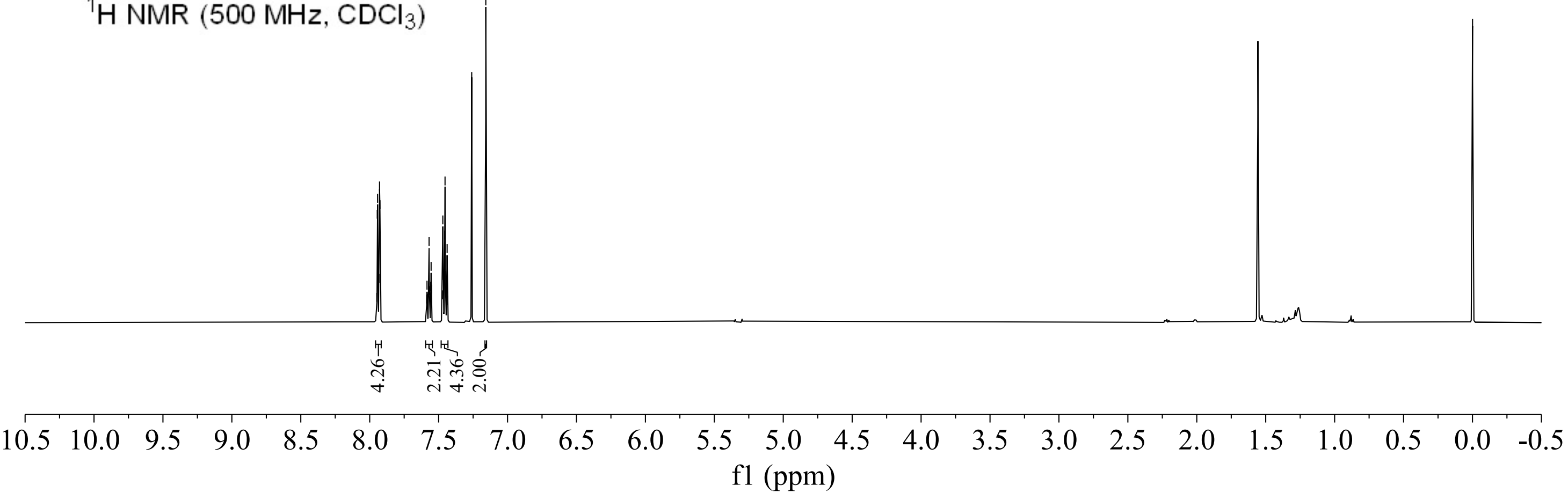



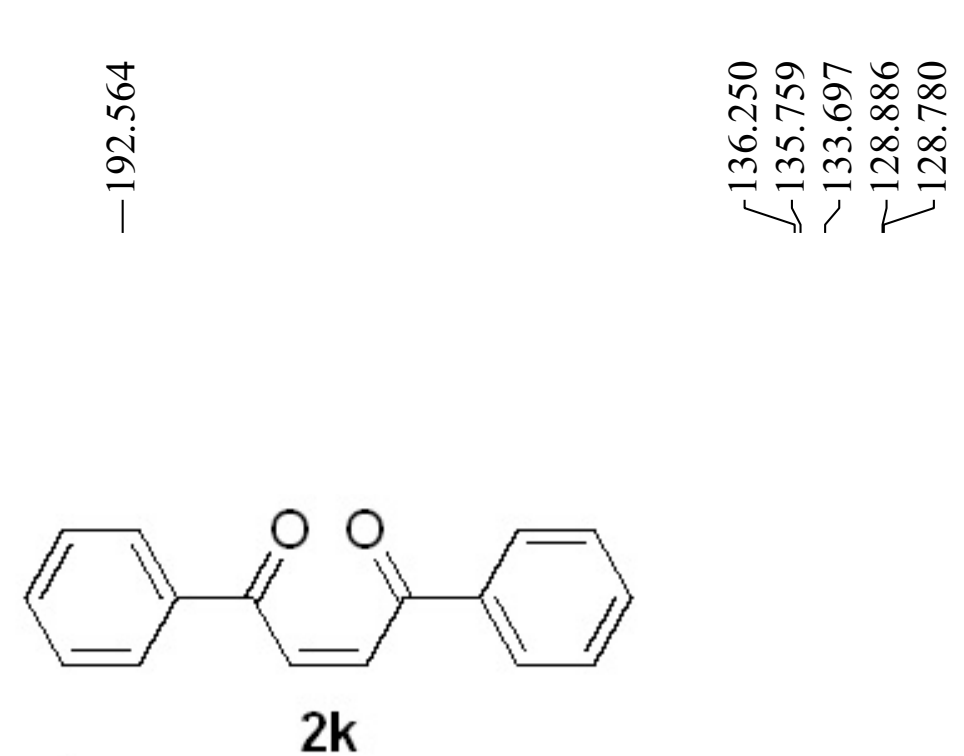

${ }^{13} \mathrm{C}\left\{{ }^{1} \mathrm{H}\right\} \operatorname{NMR}\left(126 \mathrm{MHz}, \mathrm{CDCl}_{3}\right)$

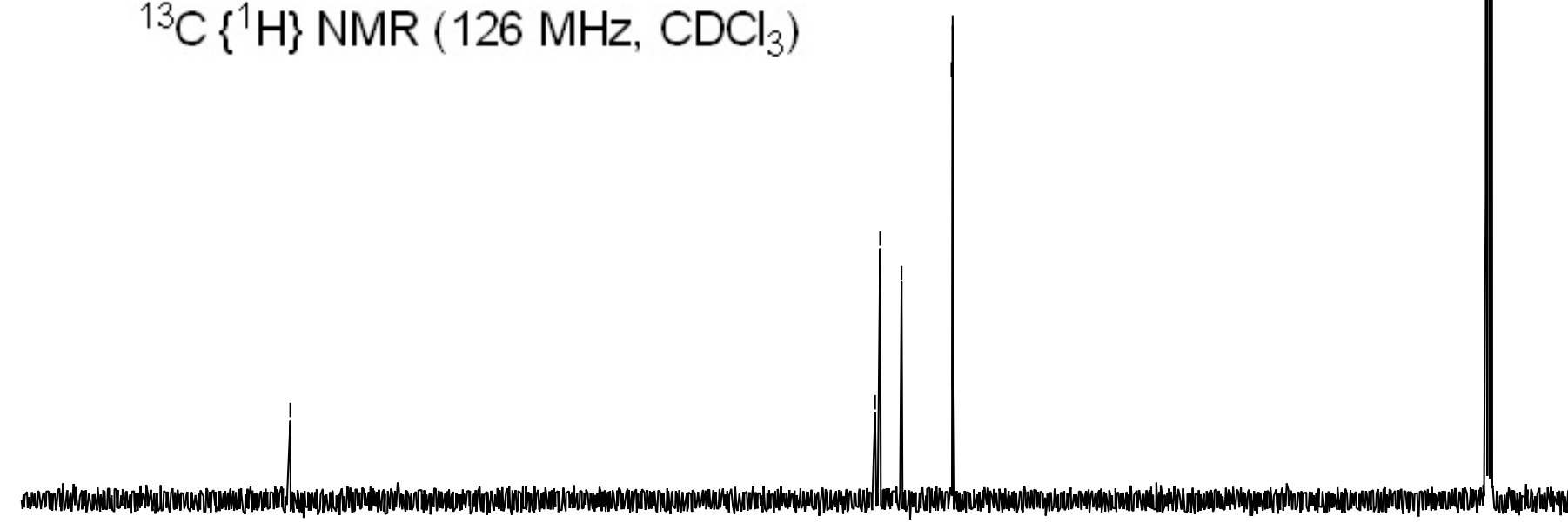

\begin{tabular}{rllllllllllllllllllllll}
\hline 210 & 200 & 190 & 180 & 170 & 160 & 150 & 140 & 130 & 120 & 110 & 100 & 90 & 80 & 70 & 60 & 50 & 40 & 30 & 20 & 10 & 0 & -10
\end{tabular}



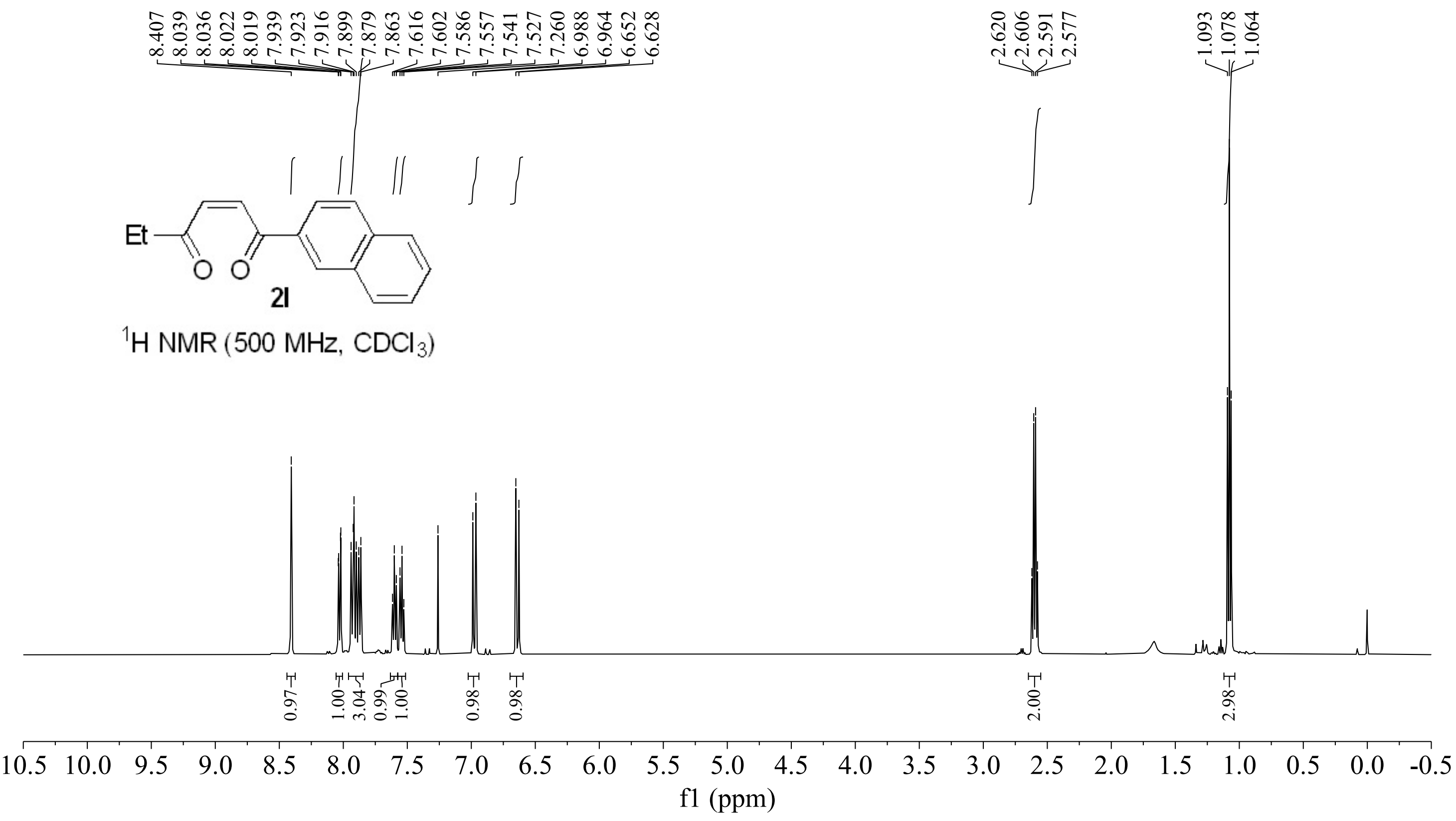


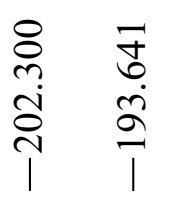

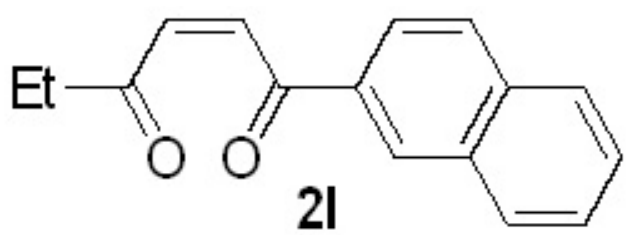

${ }^{13} \mathrm{C}\left\{{ }^{1} \mathrm{H}\right\} \operatorname{NMR}\left(126 \mathrm{MHz}, \mathrm{CDCl}_{3}\right)$

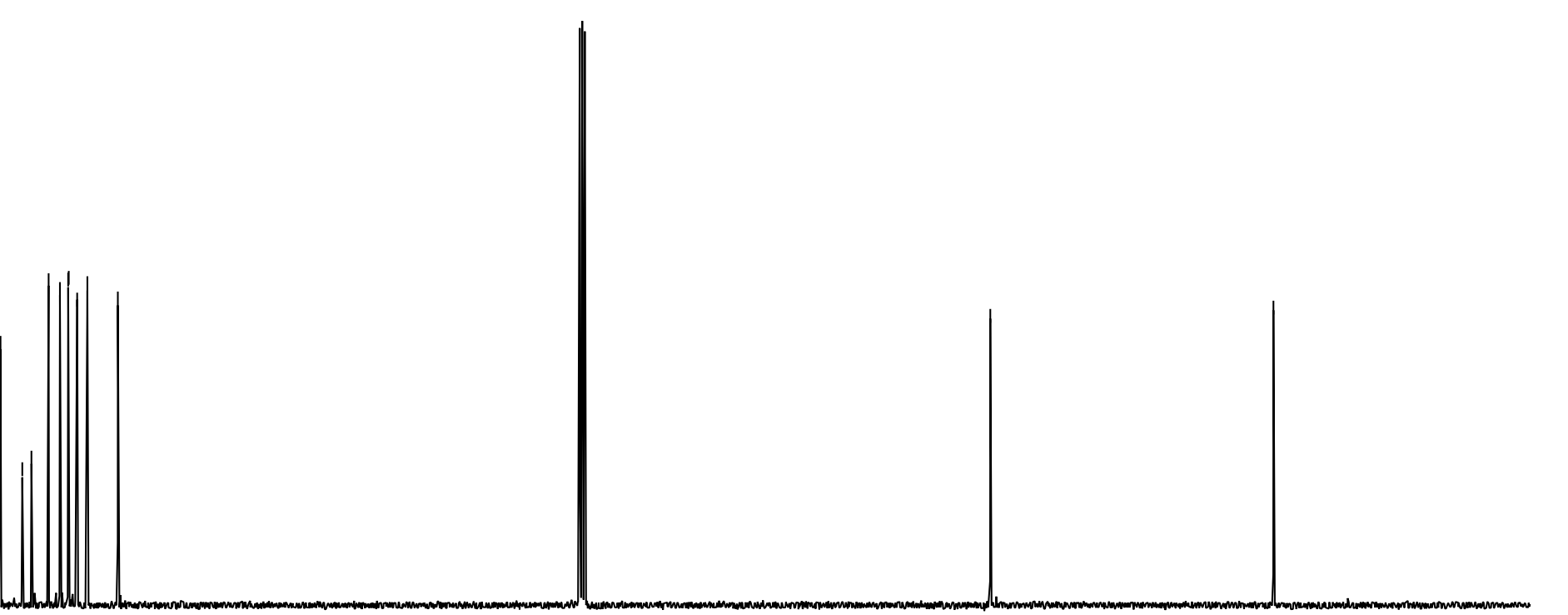

$\begin{array}{lllllllllllll}210 & 200 & 190 & 180 & 170 & 160 & 150 & 140 & 130 & 120 & 110 & 100 & 90\end{array}$ 
<smiles>CCC(=O)/C=C\C(=O)c1ccccn1</smiles>

$2 m: 3 m=1.02 /(1.02+3)=75: 25$

${ }^{1} \mathrm{H}$ NMR $\left(400 \mathrm{MHz}, \mathrm{CDCl}_{3}\right)$

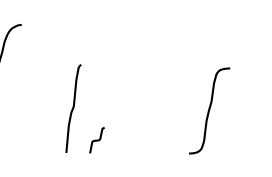




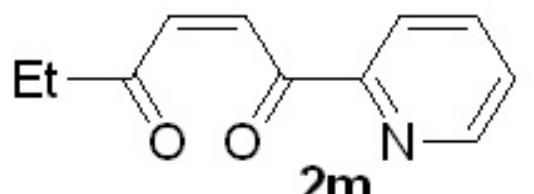

${ }^{1} \mathrm{H}$ NMR $\left(400 \mathrm{MHz}, \mathrm{CDCl}_{3}\right)$

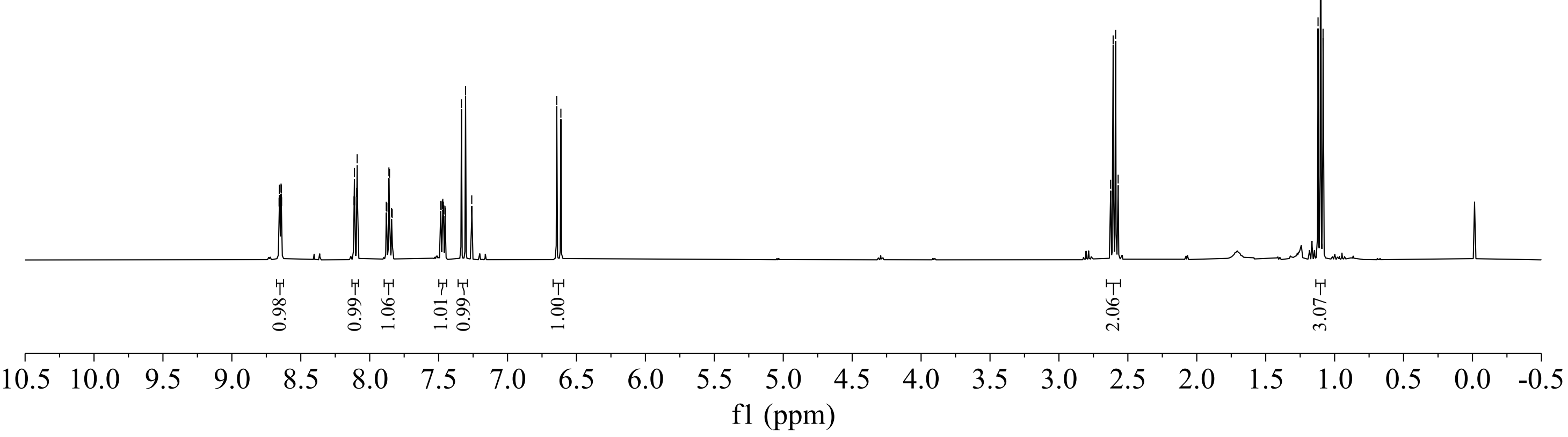




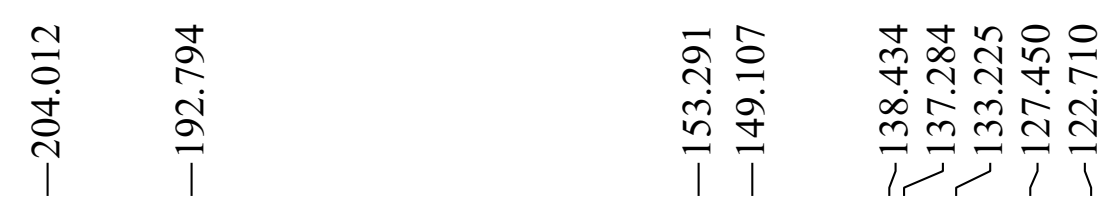<smiles>CCC(=O)/C=C\C(=O)c1ccccn1</smiles>

${ }^{13} \mathrm{C}\left\{{ }^{1} \mathrm{H}\right\} \operatorname{NMR}\left(101 \mathrm{MHz}, \mathrm{CDCl}_{3}\right)$
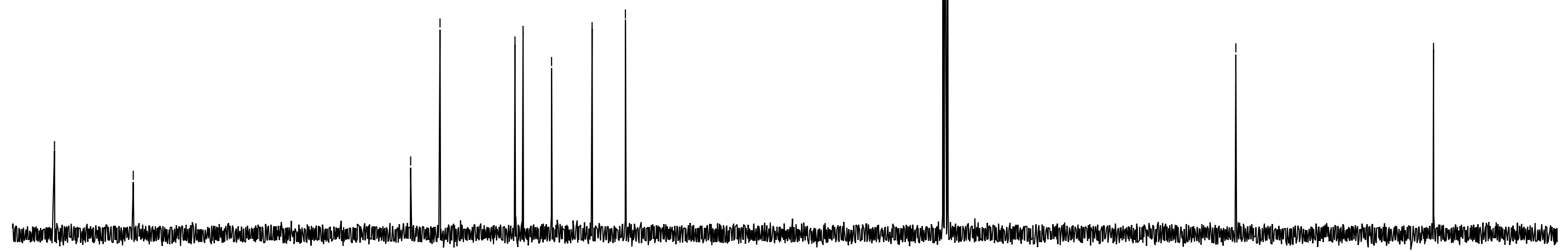

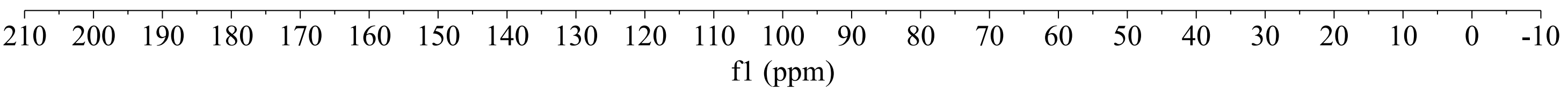




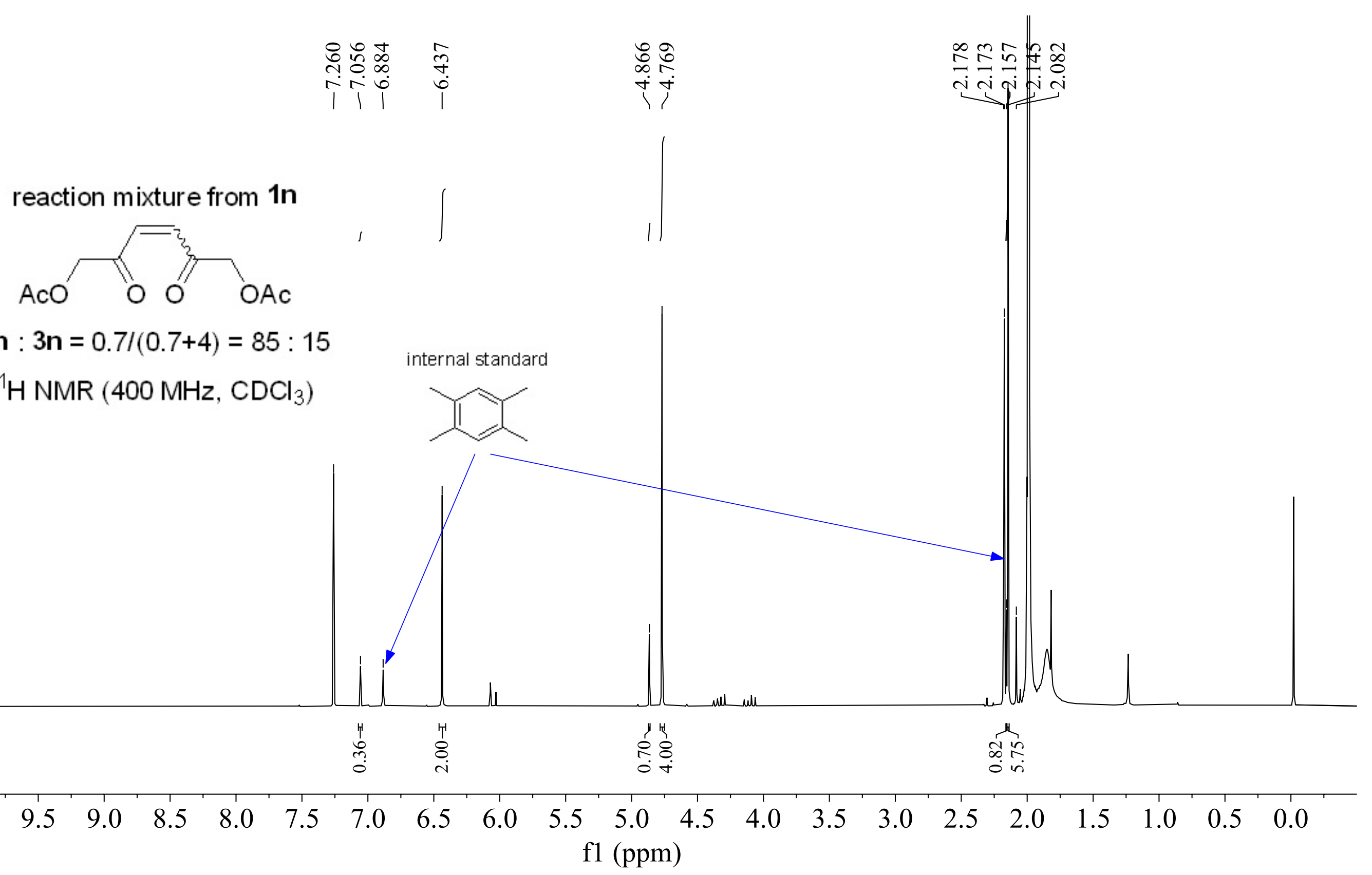




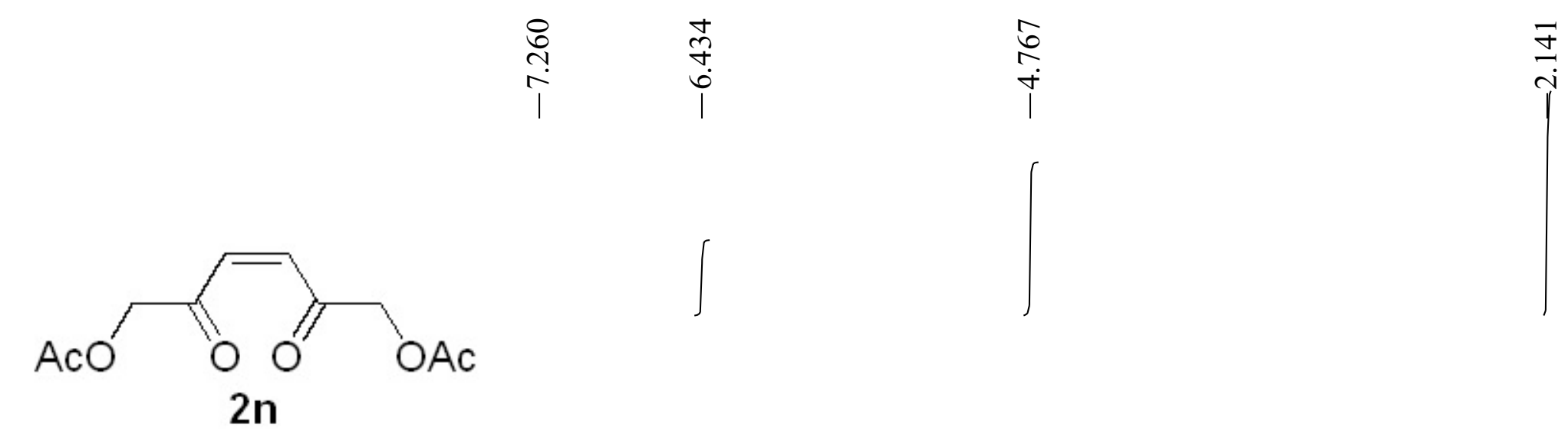

${ }^{1} \mathrm{H}$ NMR (400 MHz, $\mathrm{CDCl}_{3}$ )

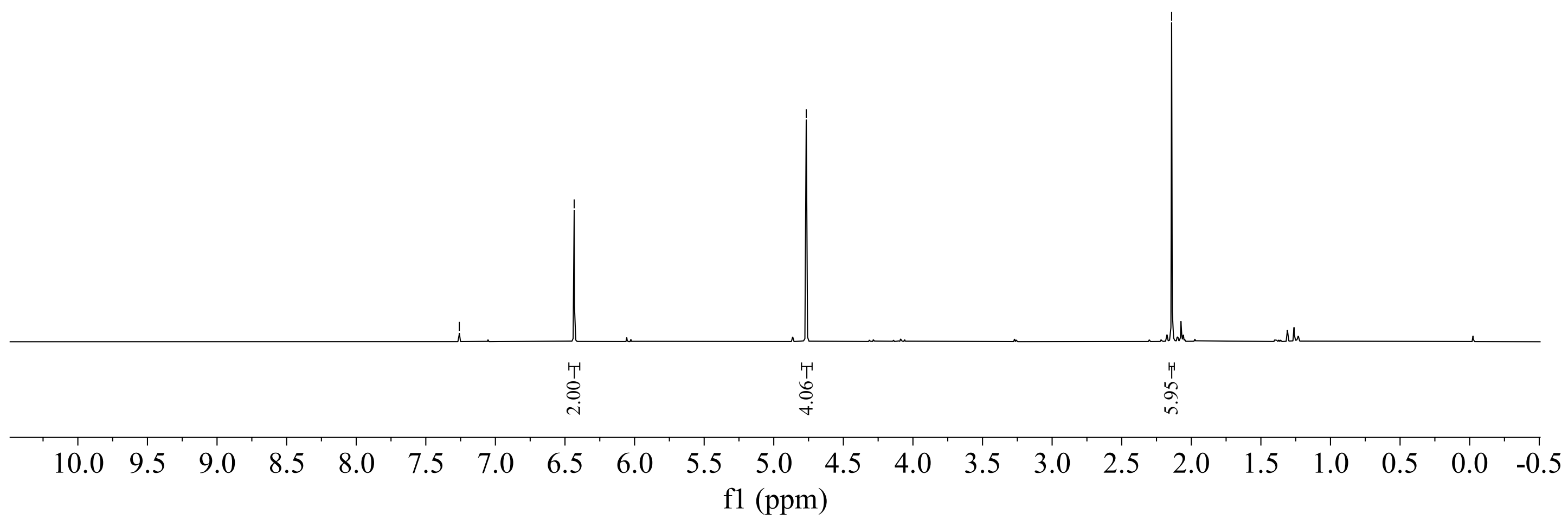




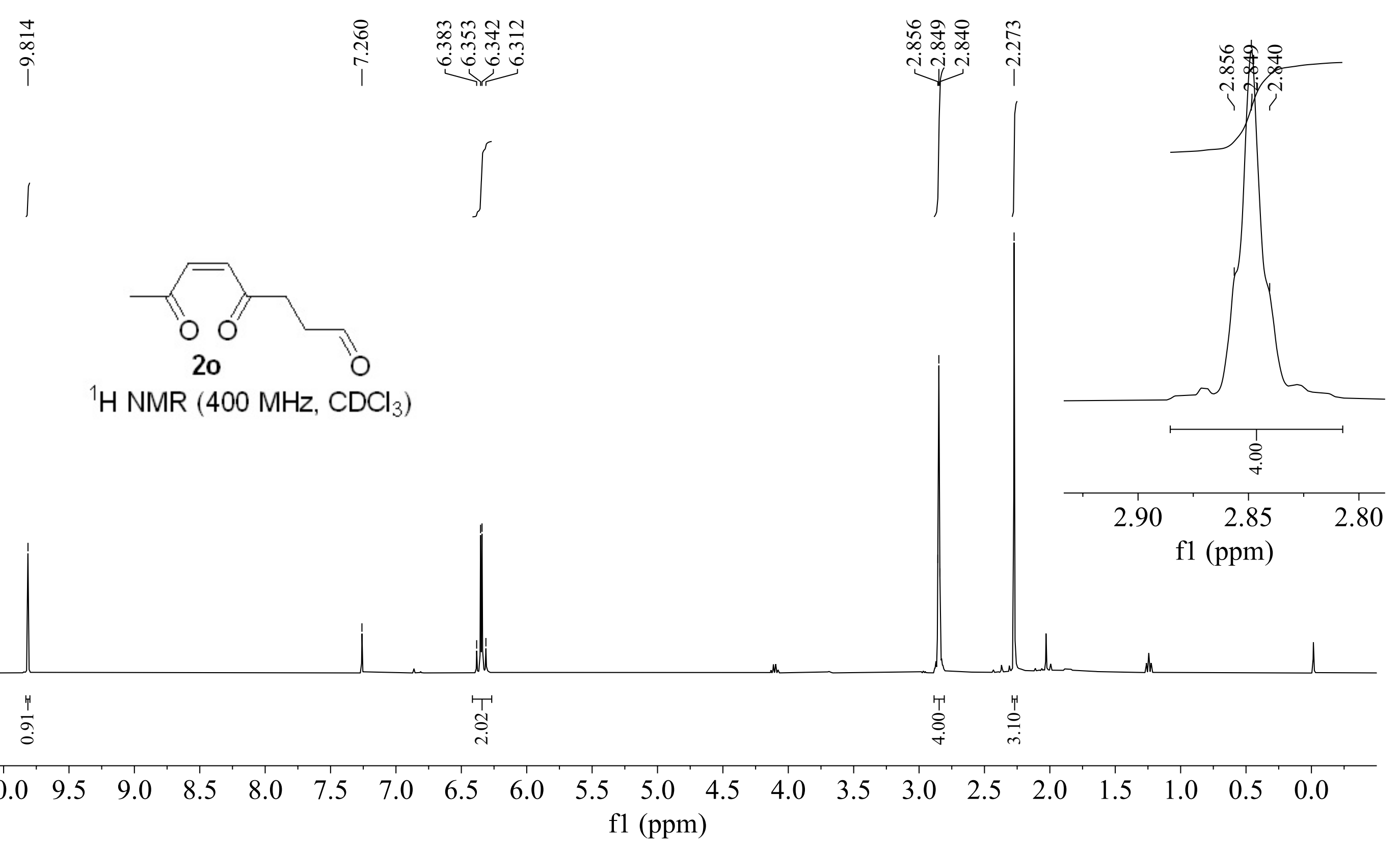




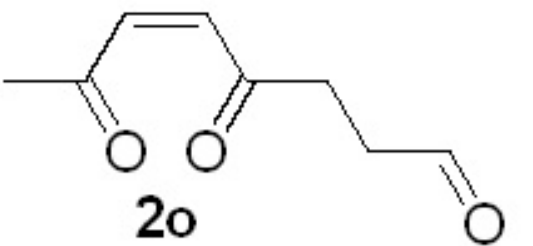

${ }^{13} \mathrm{C}\left\{{ }^{1} \mathrm{H}\right\} \operatorname{NMR}\left(101 \mathrm{MHz}, \mathrm{CDCl}_{3}\right)$

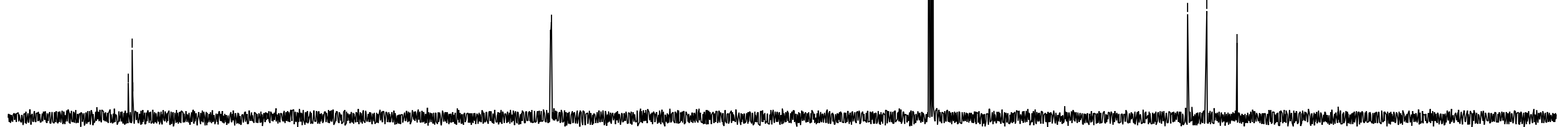

$\begin{array}{rllllllllllllllllllllll}210 & 200 & 190 & 180 & 170 & 160 & 150 & 140 & 130 & 120 & 110 & 100 & 90 & 80 & 70 & 60 & 50 & 40 & 30 & 20 & 10 & 0 & -10 \\ \mathrm{fl} & (\mathrm{ppm})\end{array}$



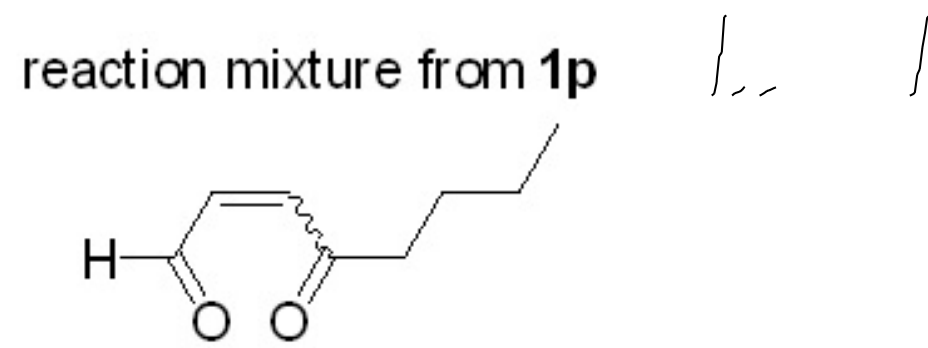

$2 p: 3 p=0.16 /(0.16+2)=93: 7$

${ }^{1} \mathrm{HNMR}\left(500 \mathrm{MHz}, \mathrm{CDCl}_{3}\right)$

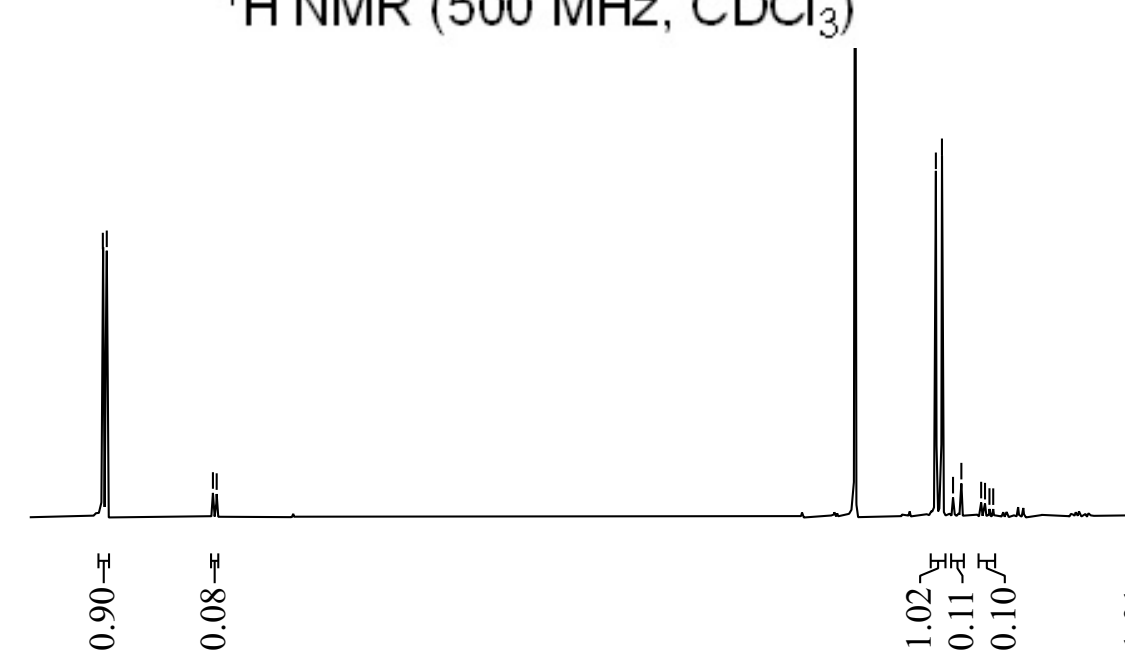

\begin{tabular}{lllllllllllllllllllllll}
\hline 10.5 & 10.0 & 9.5 & 9.0 & 8.5 & 8.0 & 7.5 & 7.0 & 6.5 & 6.0 & 5.5 & 5.0 & 4.5 & 4.0 & 3.5 & 3.0 & 2.5 & 2.0 & 1.5 & 1.0 & 0.5 & 0.0 & -0.5 \\
& & & & & &
\end{tabular} 

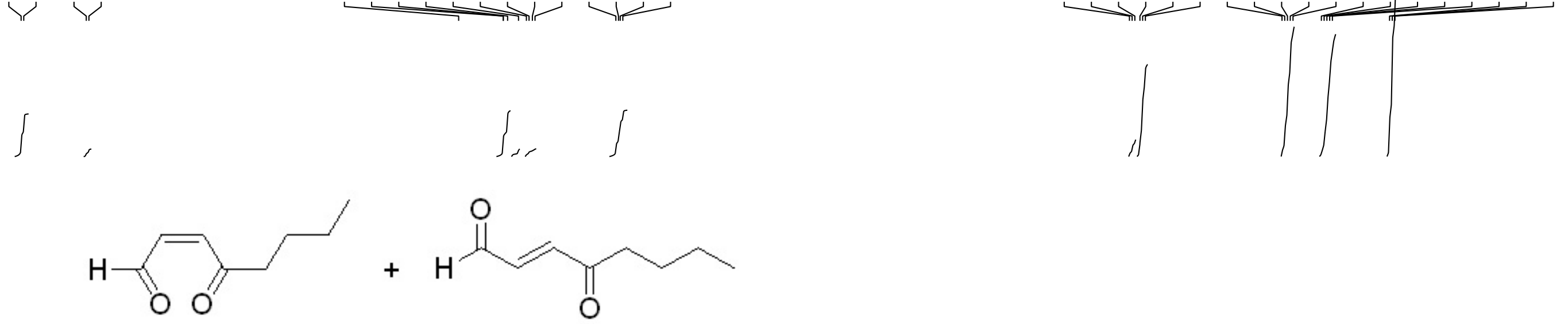

$2 p: 3 p=85: 15$

${ }^{1} \mathrm{H} \mathrm{NMR}\left(400 \mathrm{MHz}, \mathrm{CDCl}_{3}\right)$

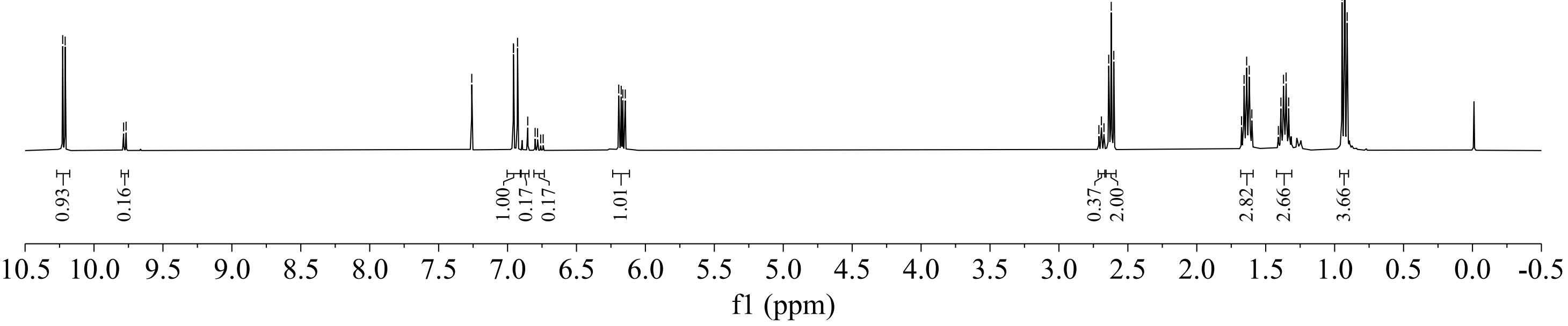




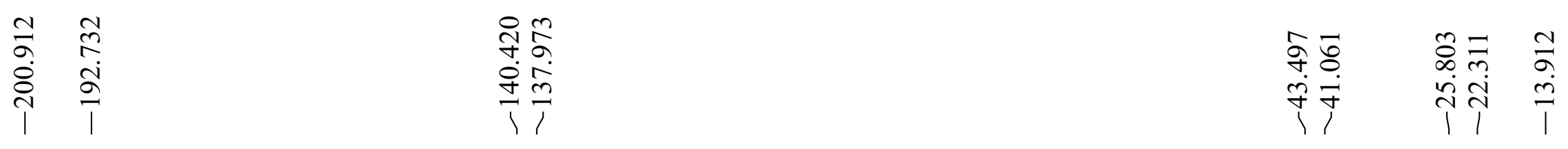<smiles>CCCCC(=O)/C=C\C=O</smiles>

$2 p+3 p$

${ }^{13} \mathrm{C}\left\{{ }^{1} \mathrm{H}\right\}$ NMR $\left(400 \mathrm{MHz}, \mathrm{CDCl}_{3}\right)$

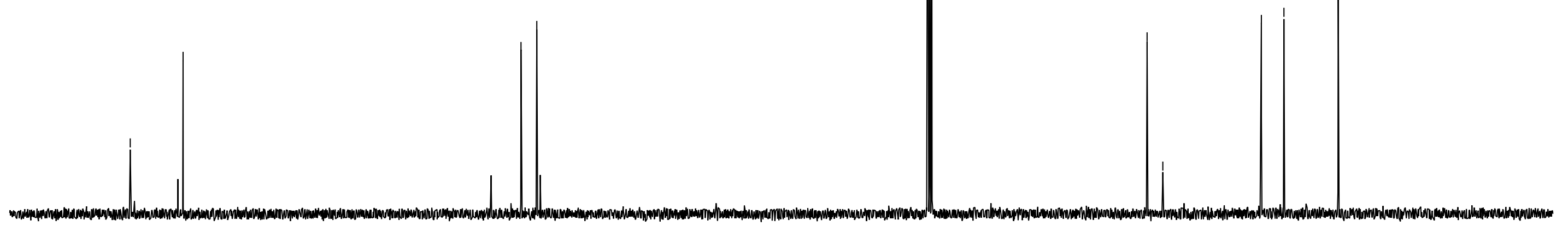

$\begin{array}{rllllllllllllllllllllll}210 & 200 & 190 & 180 & 170 & 160 & 150 & 140 & 130 & 120 & 110 & 100 & 90 & 80 & 70 & 60 & 50 & 40 & 30 & 20 & 10 & 0 & -10 \\ \mathrm{fl} & (\mathrm{ppm})\end{array}$




\section{reaction mixture from $\mathbf{1 q}$}

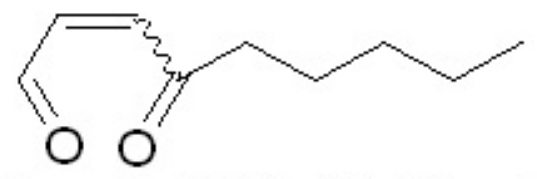

$2 q: 3 q=0.15 /(0.15+2)=93: 7$

${ }^{1} \mathrm{H}$ NMR $\left(400 \mathrm{MHz}, \mathrm{CDCl}_{3}\right)$

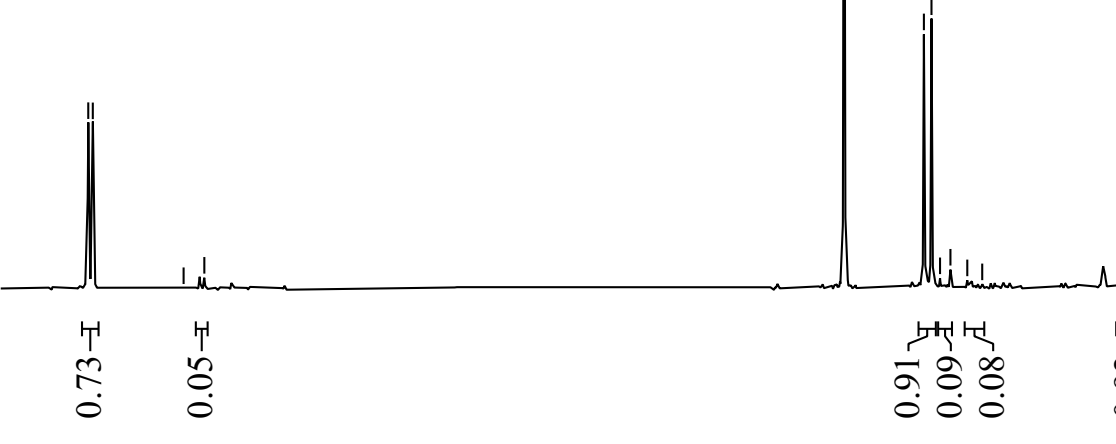

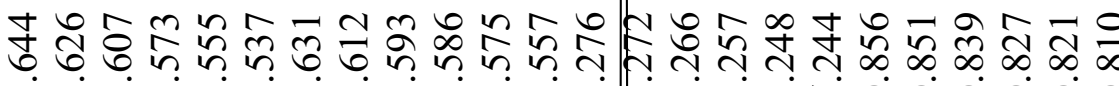
- $-1-110$ 0 0000

$$
\begin{array}{llllllllllllllllllllll}
10.0 & 9.5 & 9.0 & 8.5 & 8.0 & 7.5 & 7.0 & 6.5 & 6.0 & 5.5 & 5.0 & 4.5 & 4.0 & 3.5 & 3.0 & 2.5 & 2.0 & 1.5 & 1.0 & 0.5 & 0.0 & -0.5 \\
\mathrm{fl}(\mathrm{ppm})
\end{array}
$$




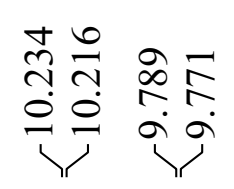

6.

$\int$

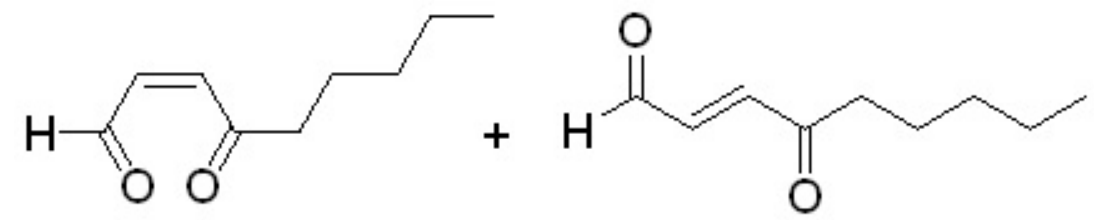

$2 q: 3 q=92: 8$

${ }^{1} \mathrm{H}$ NMR $\left(400 \mathrm{MHz}, \mathrm{CDCl}_{3}\right)$

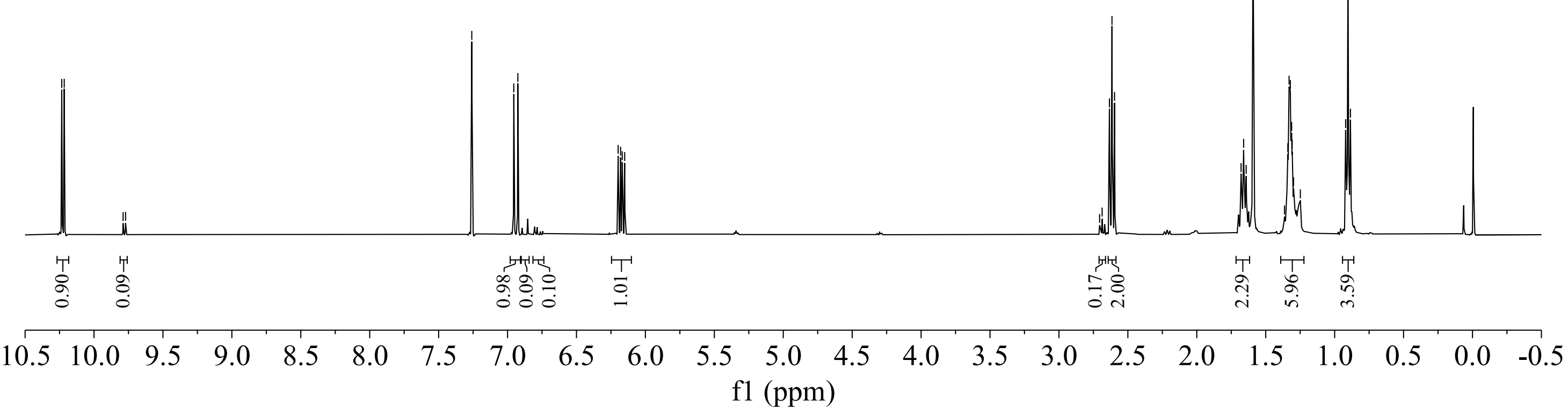




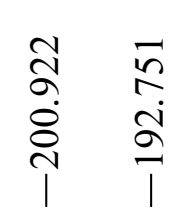

$$
\begin{aligned}
& \text { 誉京 }
\end{aligned}
$$

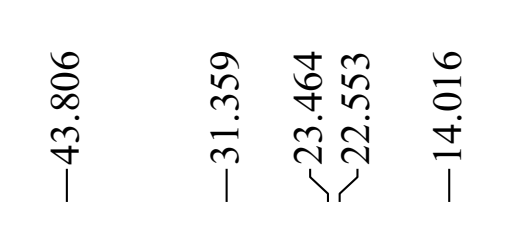

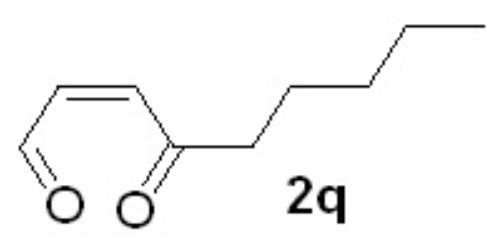

${ }^{13} \mathrm{C}$ NMR (101 MHz, $\mathrm{CDCl}_{3}$ )

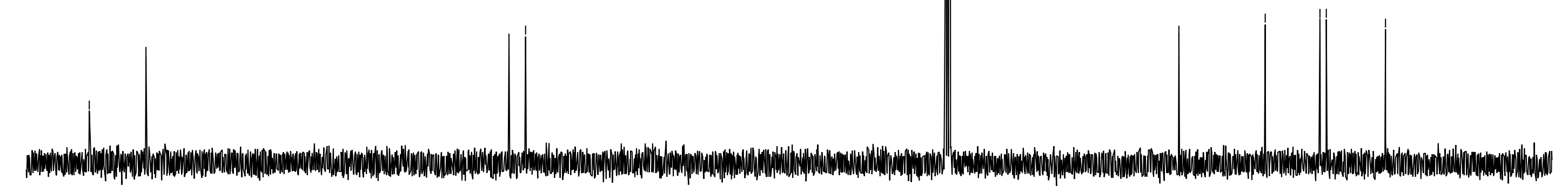

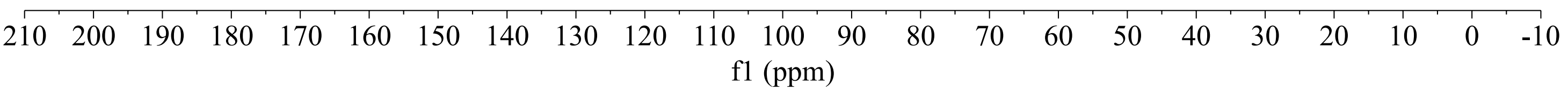




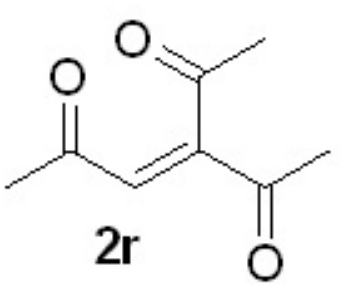

${ }^{1} \mathrm{H}$ NMR $\left(500 \mathrm{MHz}, \mathrm{CDCl}_{3}\right)$

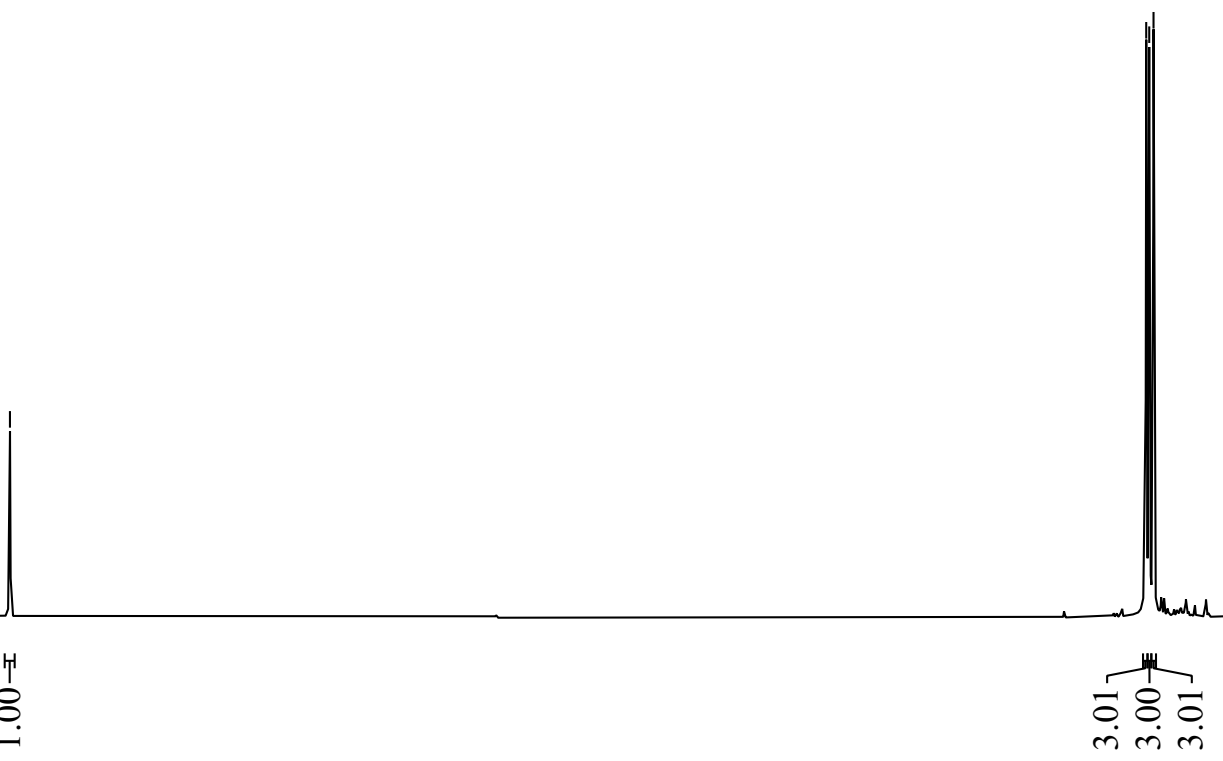

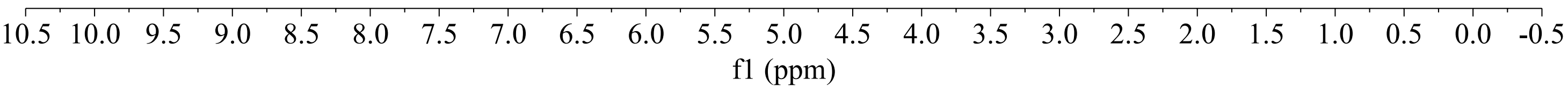




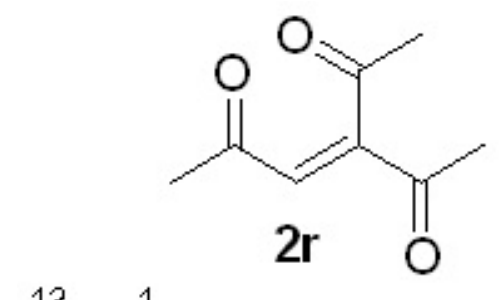

${ }^{13} \mathrm{C}\left\{{ }^{1} \mathrm{H}\right\} \mathrm{NMR}\left(126 \mathrm{MHz}, \mathrm{CDCl}_{3}\right)$

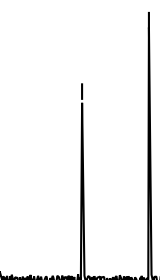

$z, \mathrm{CDCl}_{3}$

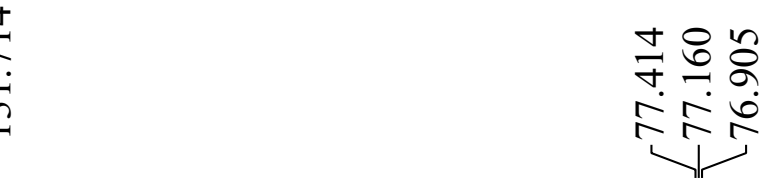

نंi

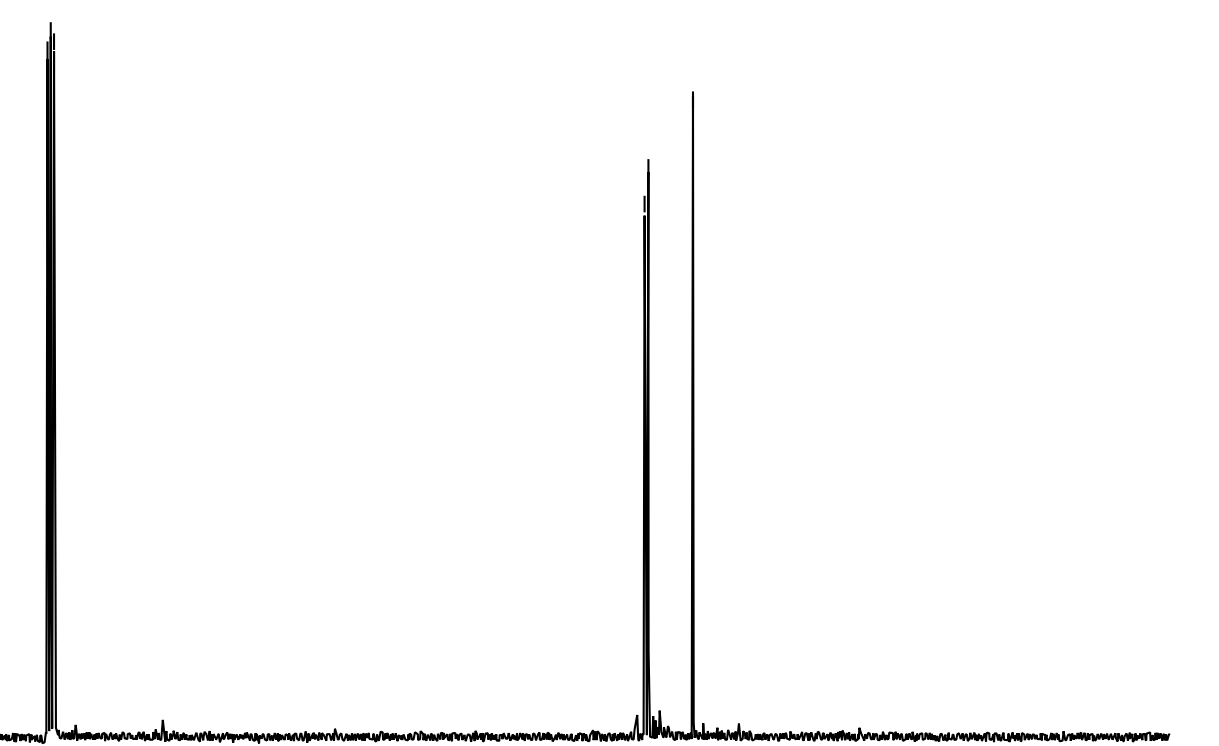

$\begin{array}{rllllllllllllllllllllllll}210 & 200 & 190 & 180 & 170 & 160 & 150 & 140 & 130 & 120 & 110 & 100 & 90 & 80 & 70 & 60 & 50 & 40 & 30 & 20 & 10 & 0 & -10\end{array}$




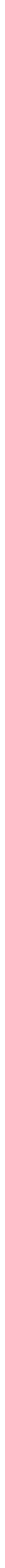




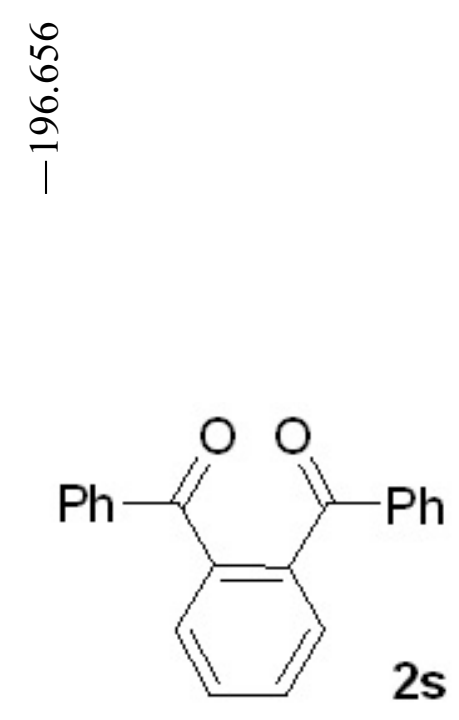

${ }^{13} \mathrm{C}\left\{{ }^{1} \mathrm{H}\right\} \mathrm{NMR}\left(126 \mathrm{MHz}, \mathrm{CDCl}_{3}\right)$

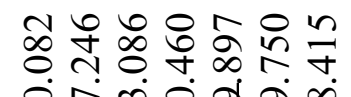

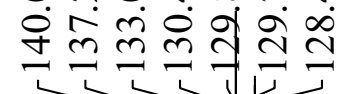

3

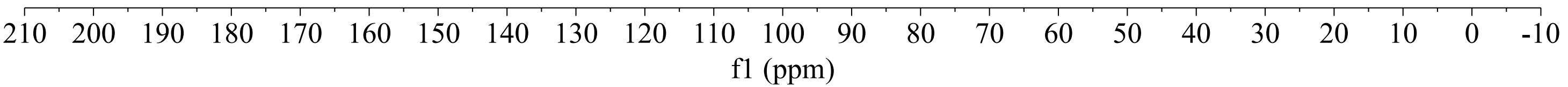




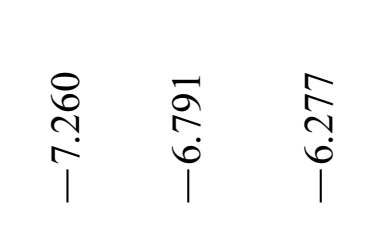

the reaction mixture from 1 t

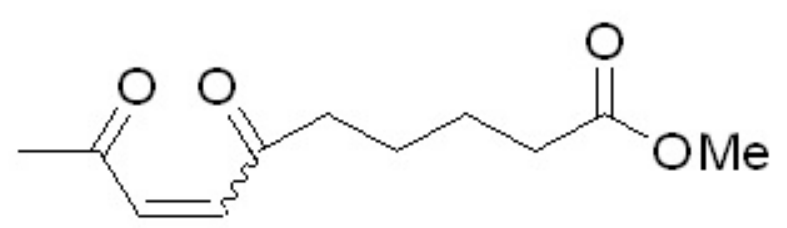

$2 t: 3 t=0.19 /(0.19+2)=91: 9$

${ }^{1} \mathrm{H}$ NMR (400 MHz, $\mathrm{CDCl}_{3}$ )
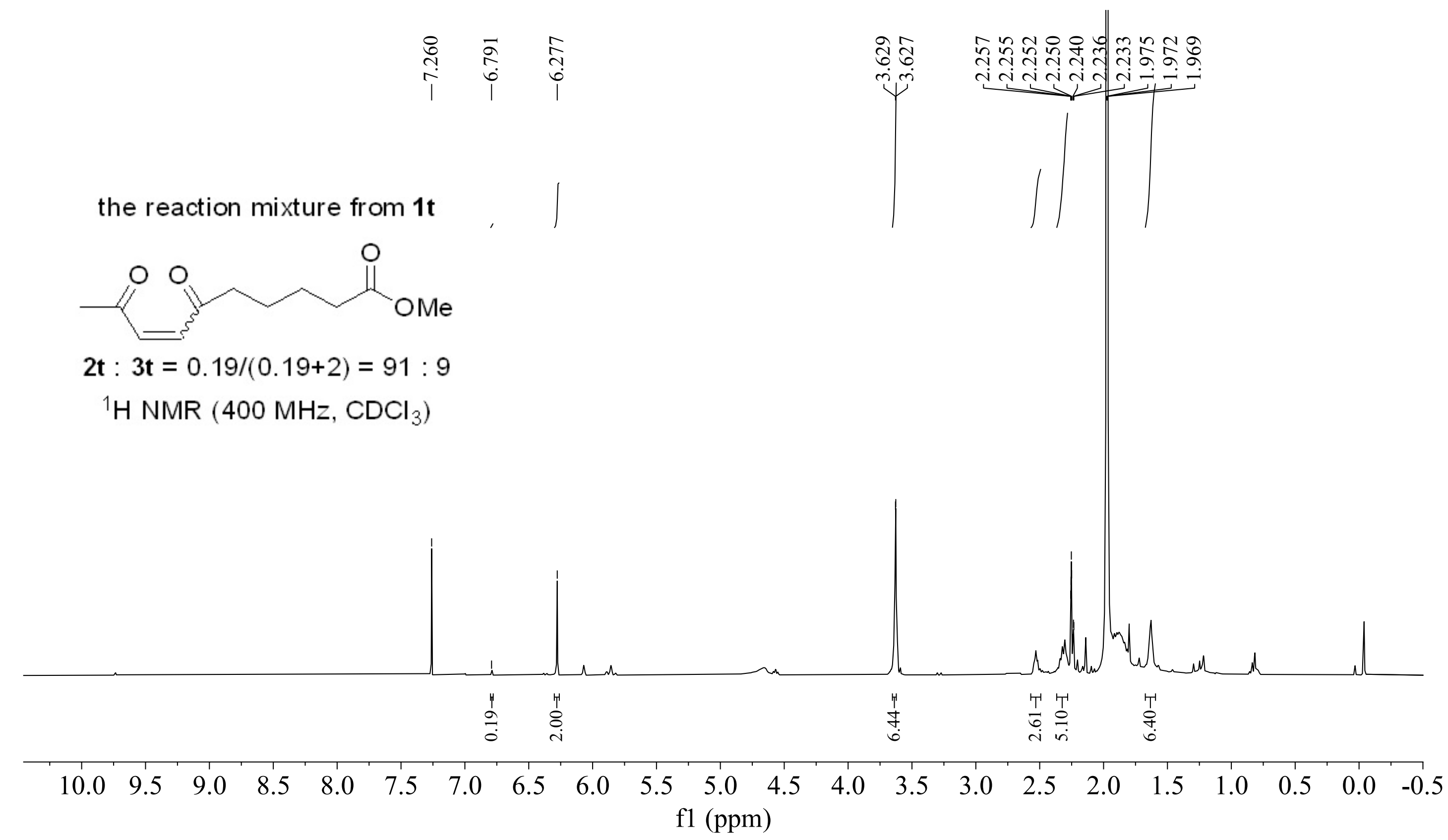

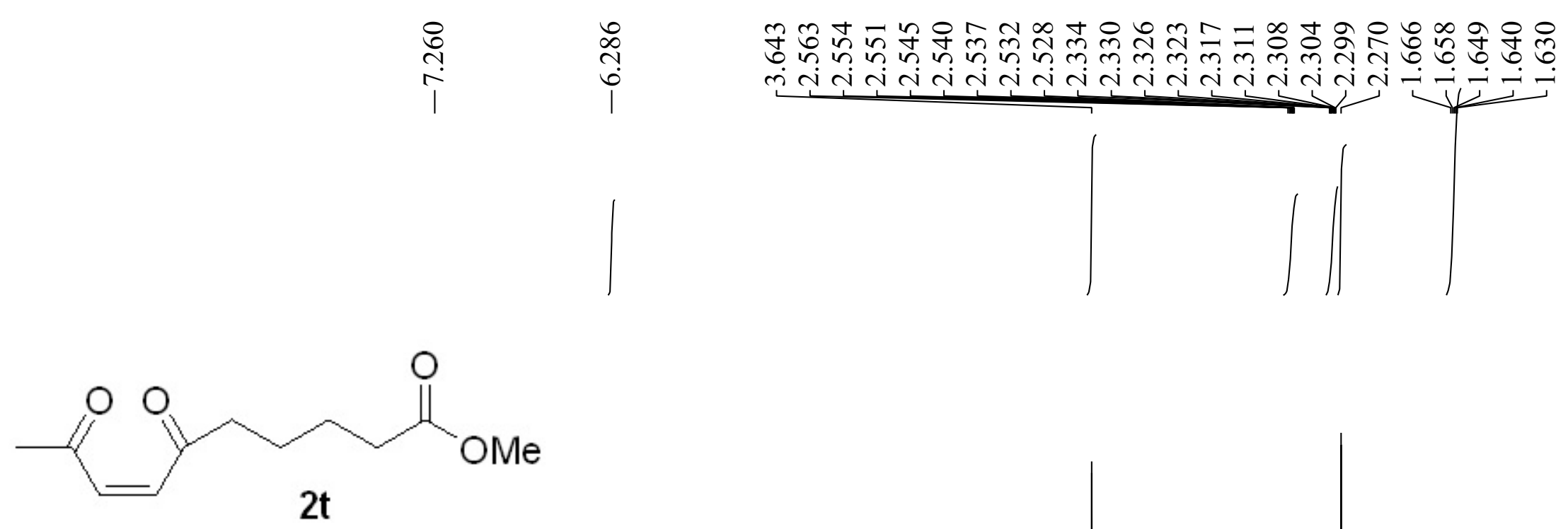

${ }^{1} \mathrm{H} \operatorname{NMR}\left(400 \mathrm{MHz}, \mathrm{CDCl}_{3}\right)$

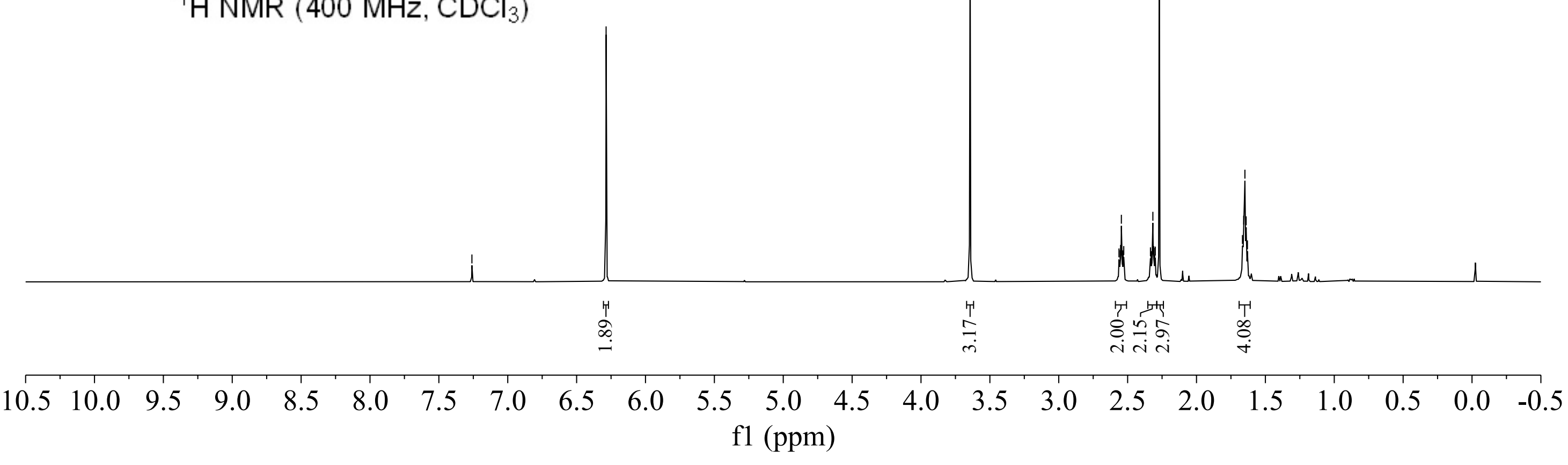


reaction mixture of $1 \mathbf{u}$

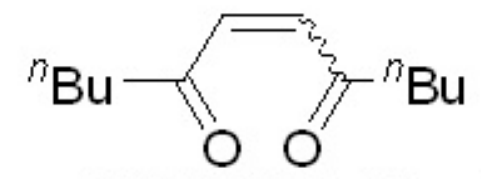

$2 \mathbf{u}: 3 \mathbf{u}=0.37 /(0.37+2)=85: 15$

${ }^{1} \mathrm{H}$ NMR (400 MHz, $\mathrm{CDCl}_{3}$ )

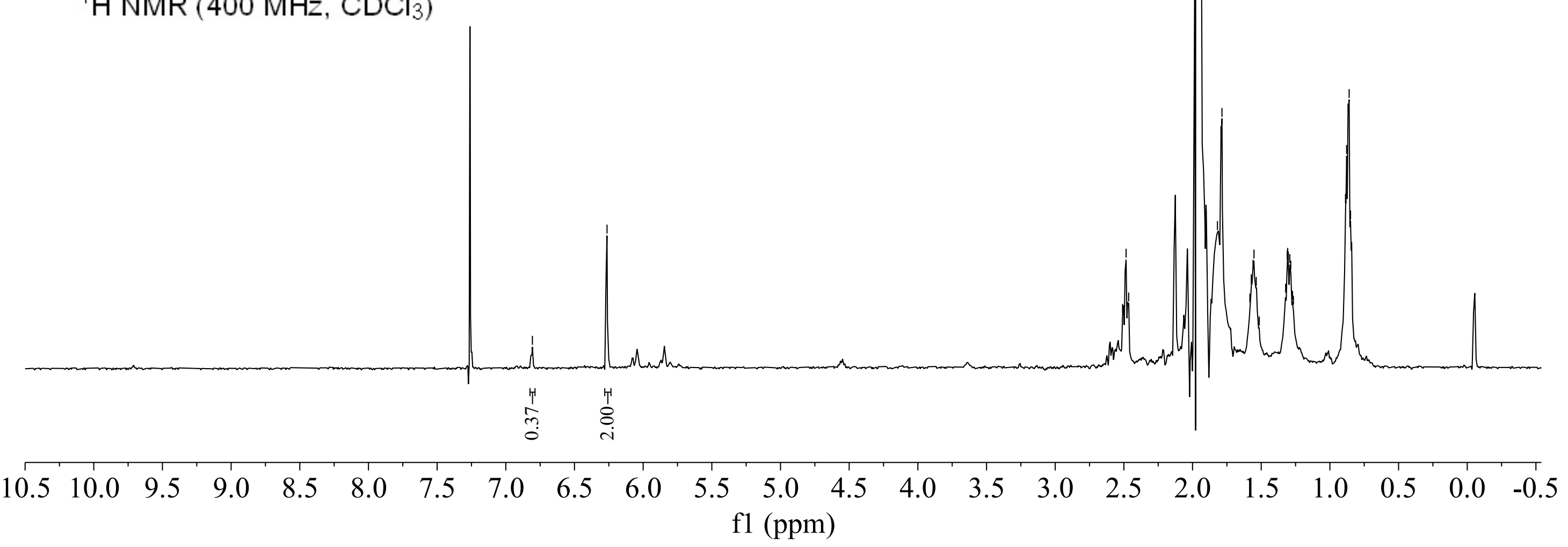




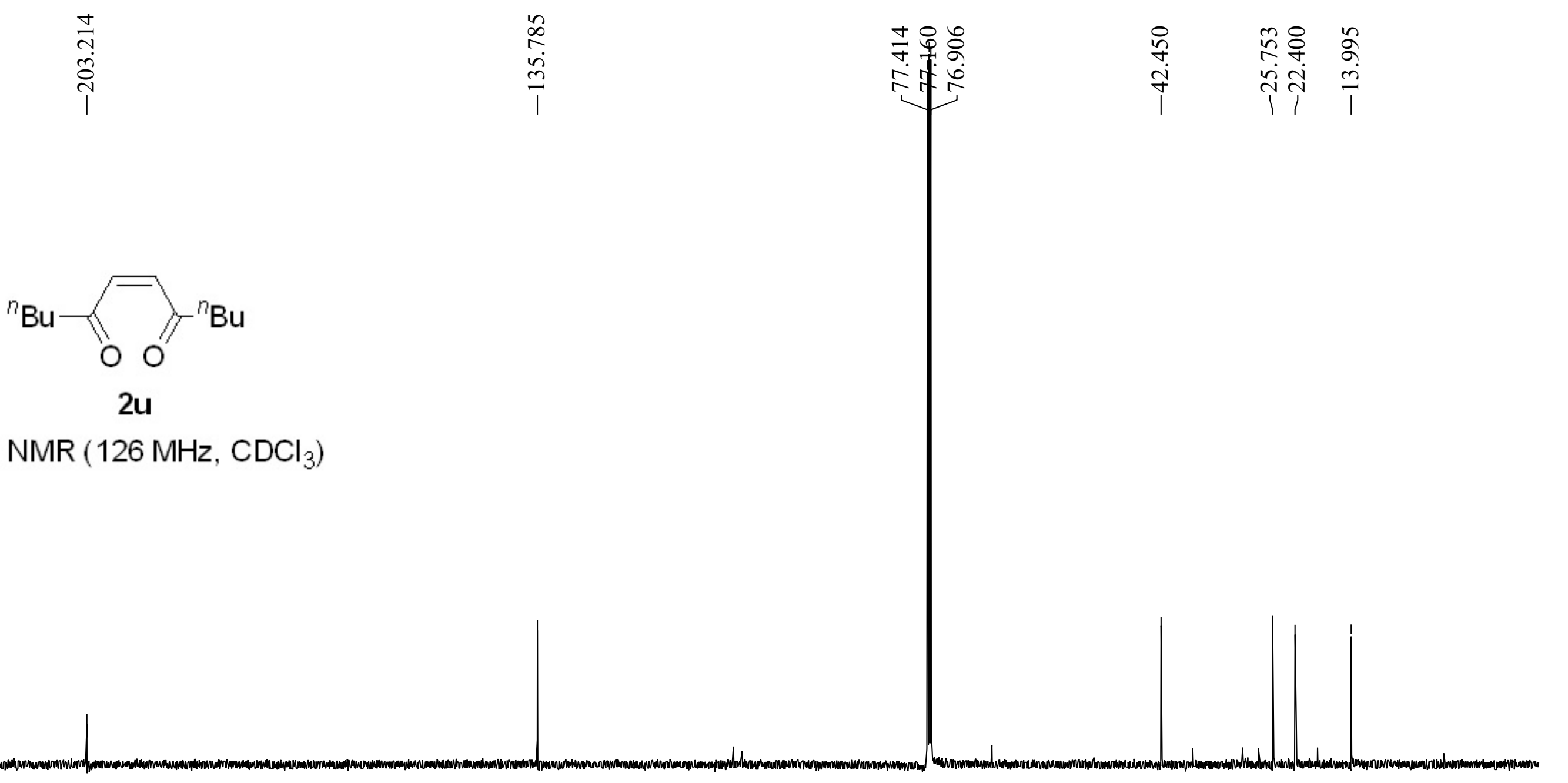

$\begin{array}{rllllllllllllllllllllllllllll}230 & 220 & 210 & 200 & 190 & 180 & 170 & 160 & 150 & 140 & 130 & 120 & 110 & 100 & 90 & 80 & 70 & 60 & 50 & 40 & 30 & 20 & 10 & 0 & -10 \\ \mathrm{f} 1(\mathrm{ppm})\end{array}$




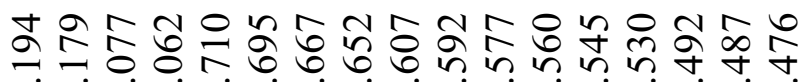

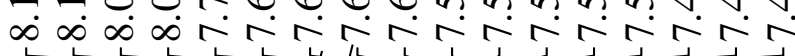

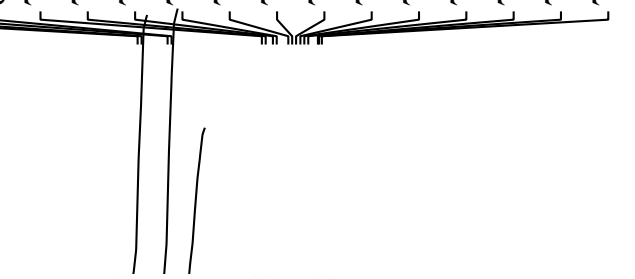

crude products

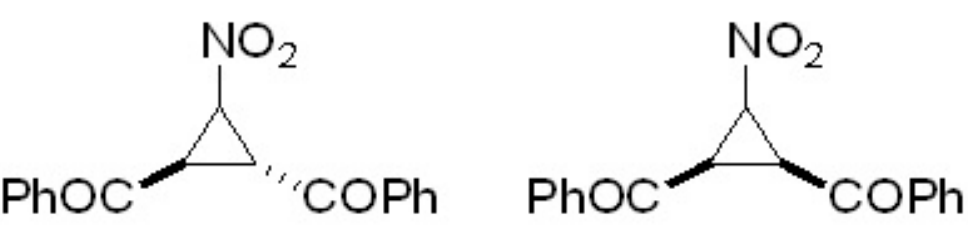

$4: 4^{\prime}=0.18 /(1+0.18)=85: 15$

${ }^{1} \mathrm{HNMR}\left(400 \mathrm{MHz}, \mathrm{CDCl}_{3}\right)$

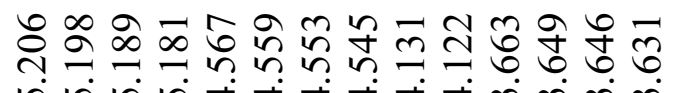

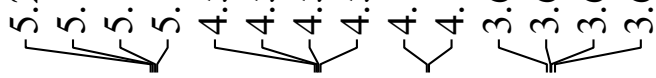

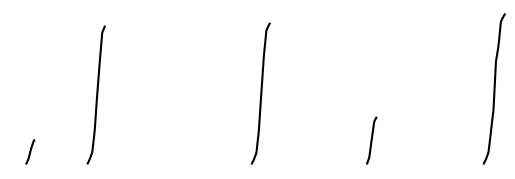

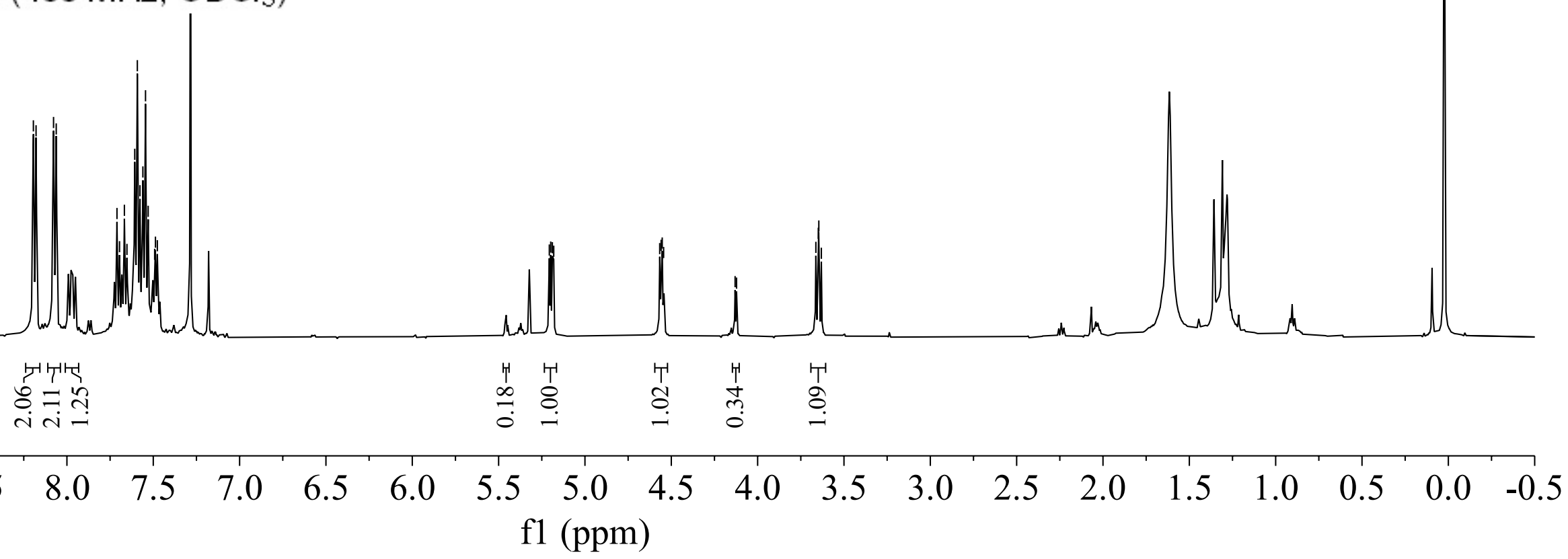




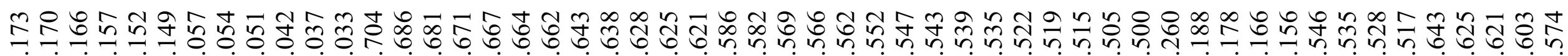

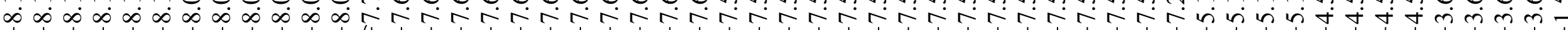

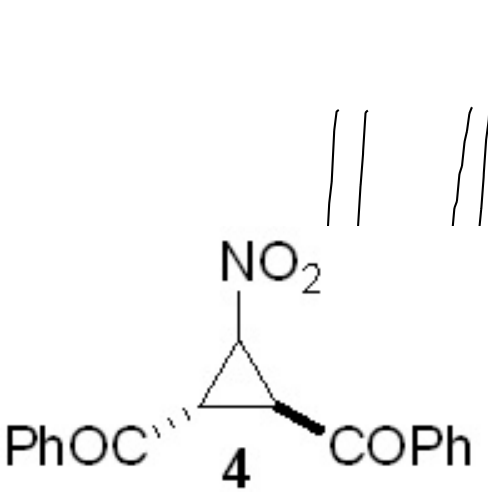

${ }^{1} \mathrm{H}$ NMR $\left(400 \mathrm{MHz}, \mathrm{CDCl}_{3}\right)$

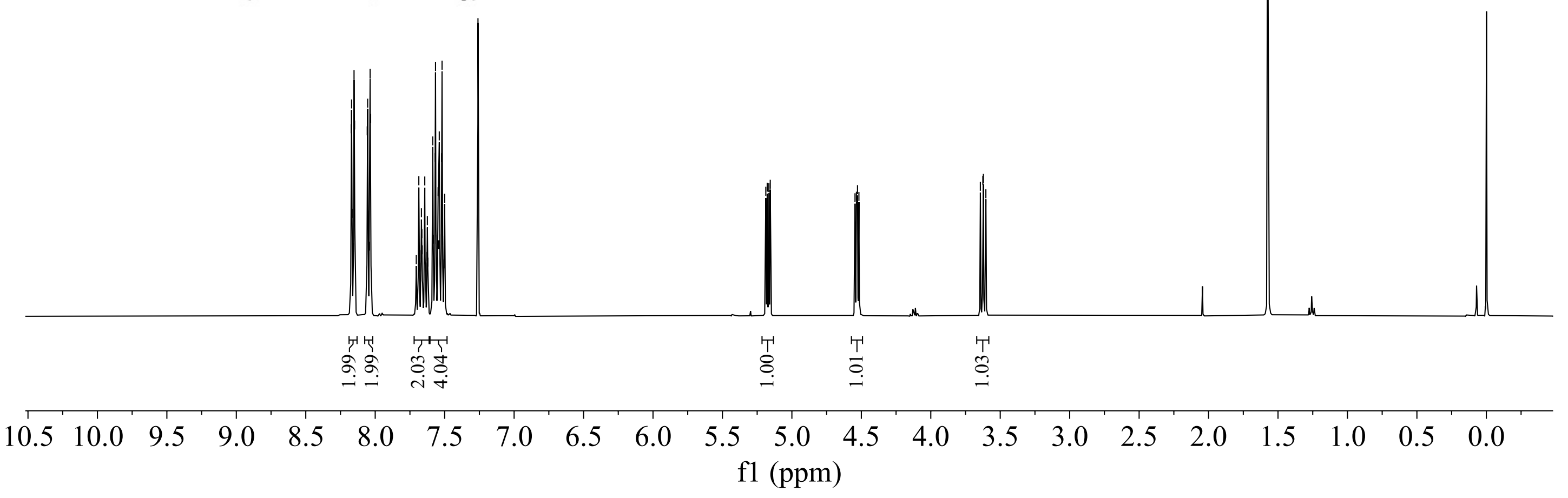




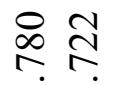

๙ $\underset{1}{\infty} \stackrel{\infty}{1}$

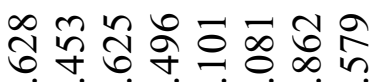

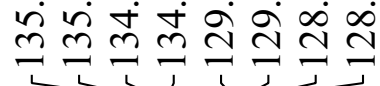<smiles>O=C(c1ccccc1)C1C([C+]([O-])c2ccccc2)C1[N+](=O)[O-]</smiles>

${ }^{13} \mathrm{C}\left\{{ }^{1} \mathrm{H}\right\} \operatorname{NMR}\left(101 \mathrm{MHz}, \mathrm{CDCl}_{3}\right)$

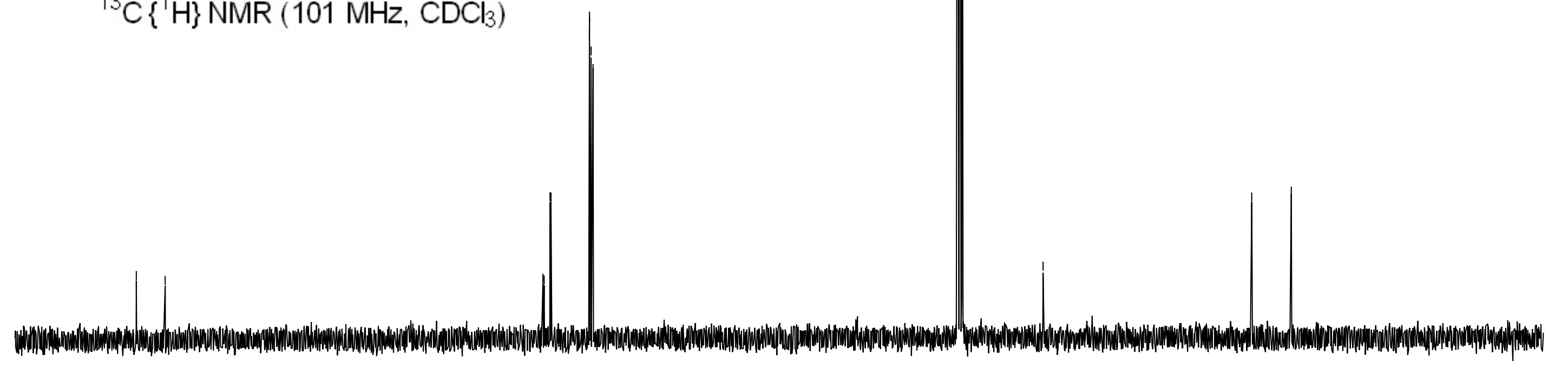

$\begin{array}{lllllllllllllllllllll}200 & 190 & 180 & 170 & 160 & 150 & 140 & 130 & 120 & \begin{array}{c}110 \\ \mathrm{f} 1(\mathrm{ppm})\end{array} & \begin{array}{c}100 \\ (\mathrm{pm})\end{array} & 80 & 70 & 60 & 50 & 40 & 30 & 20 & 10 & 0\end{array}$




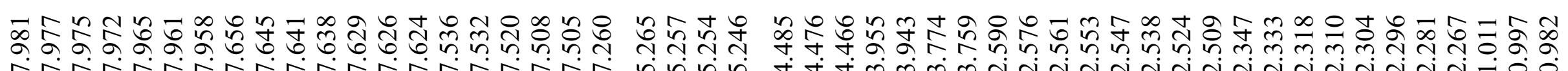

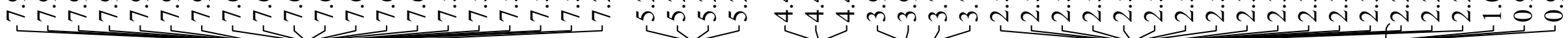
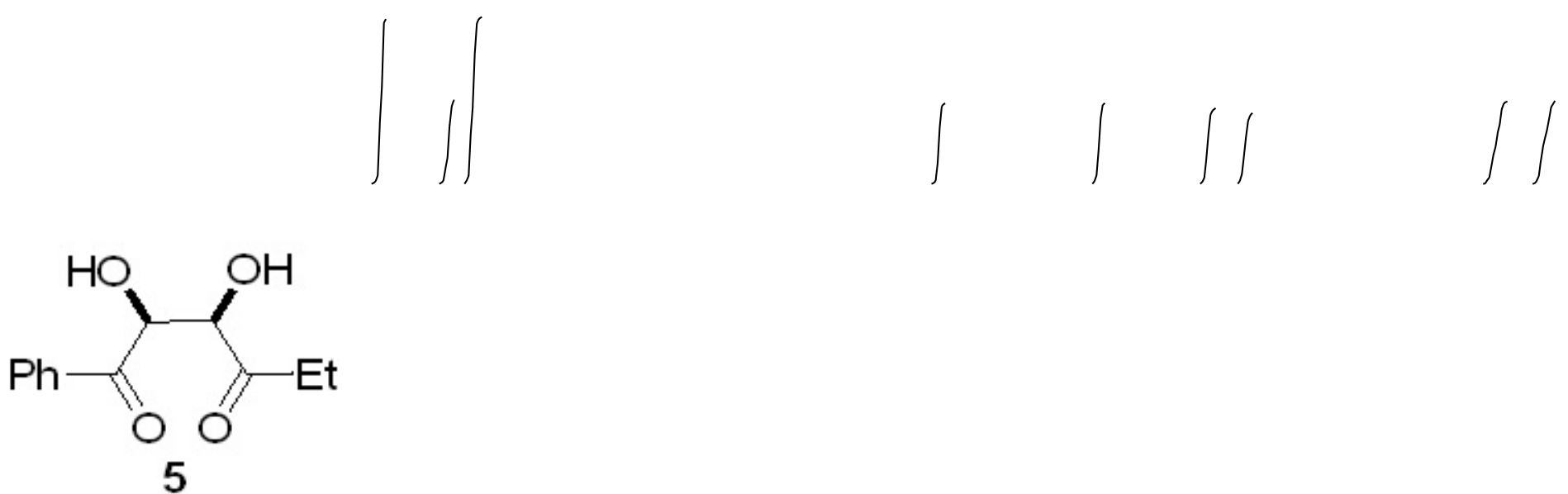

${ }^{1} \mathrm{HNMR}\left(500 \mathrm{MHz}, \mathrm{CDCl}_{3}\right)$

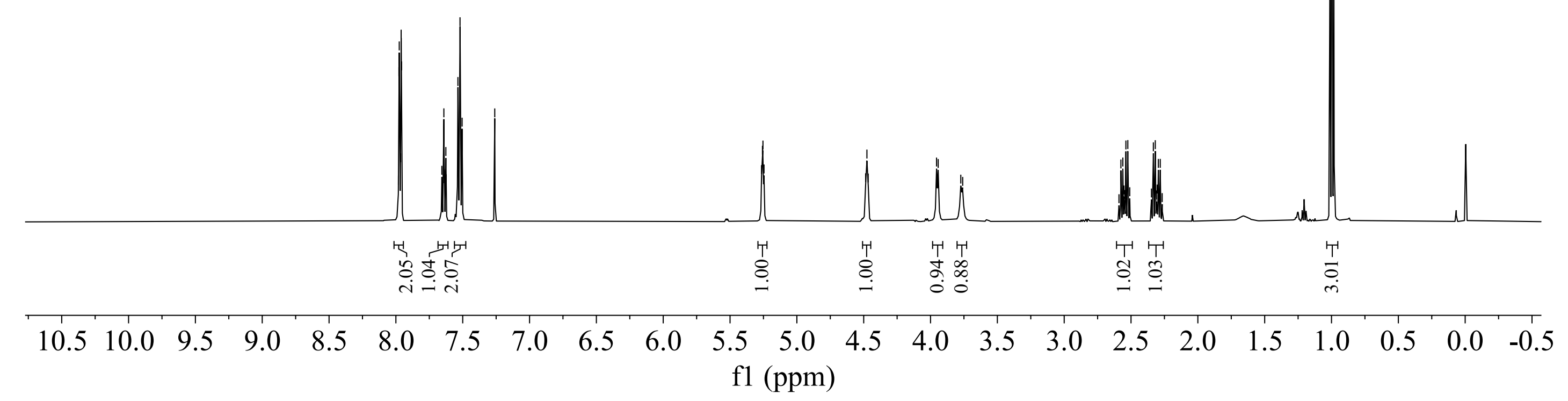




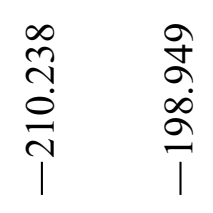

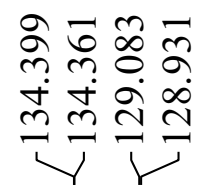

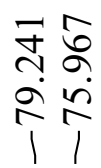

$\overrightarrow{+}$
mे
i

$\frac{0}{8}$<smiles>CCC(=O)C(O)C(O)C(=O)c1ccccc1</smiles>

${ }^{13} \mathrm{C}\left\{{ }^{1} \mathrm{H}\right\} \operatorname{NMR}\left(101 \mathrm{MHz}, \mathrm{CDCl}_{3}\right)$

Wham

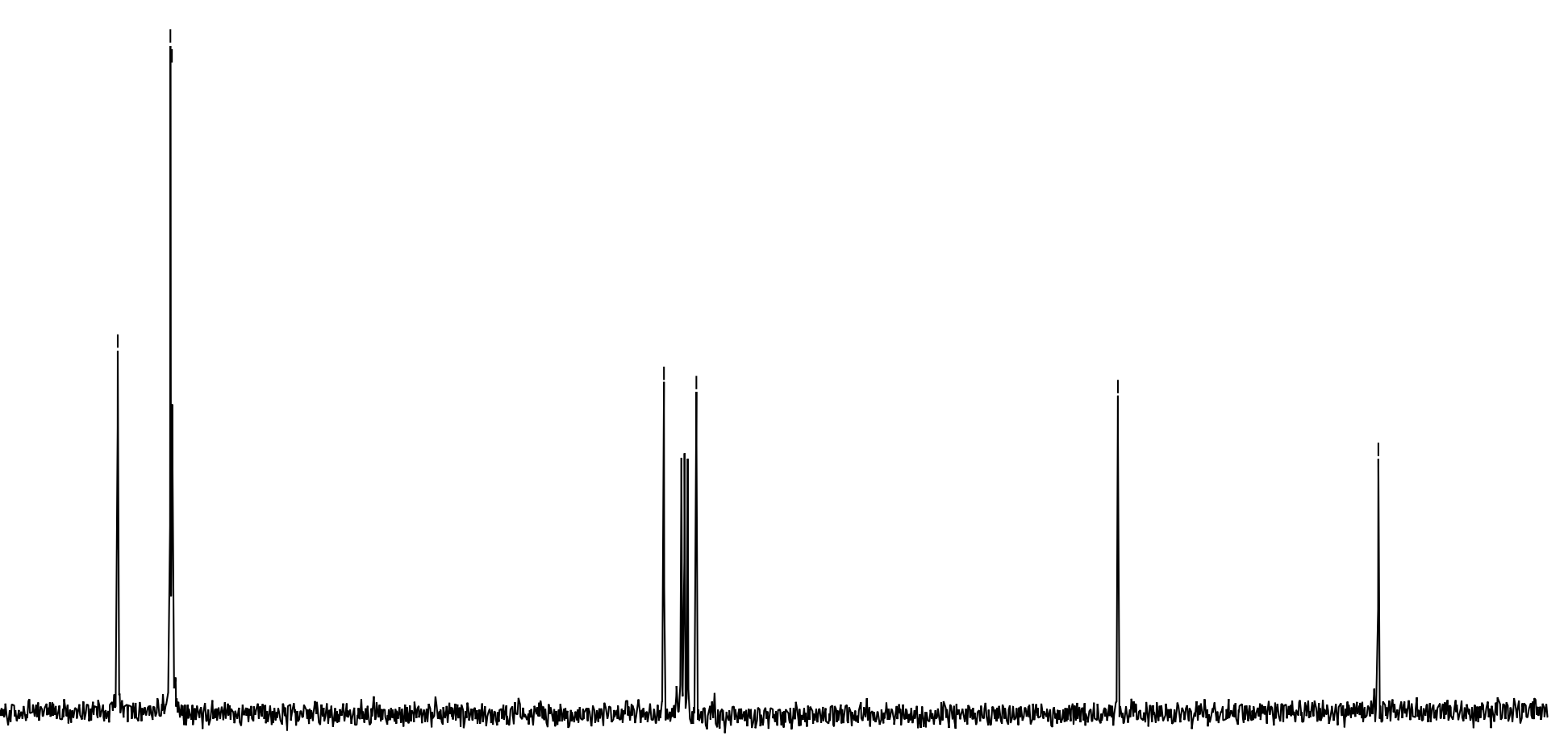

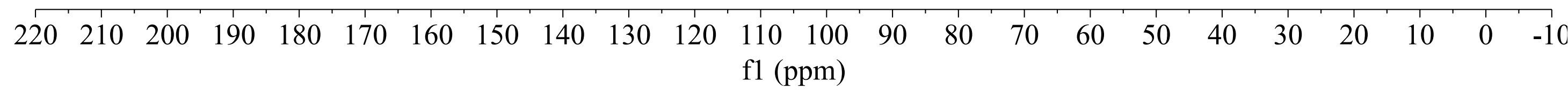




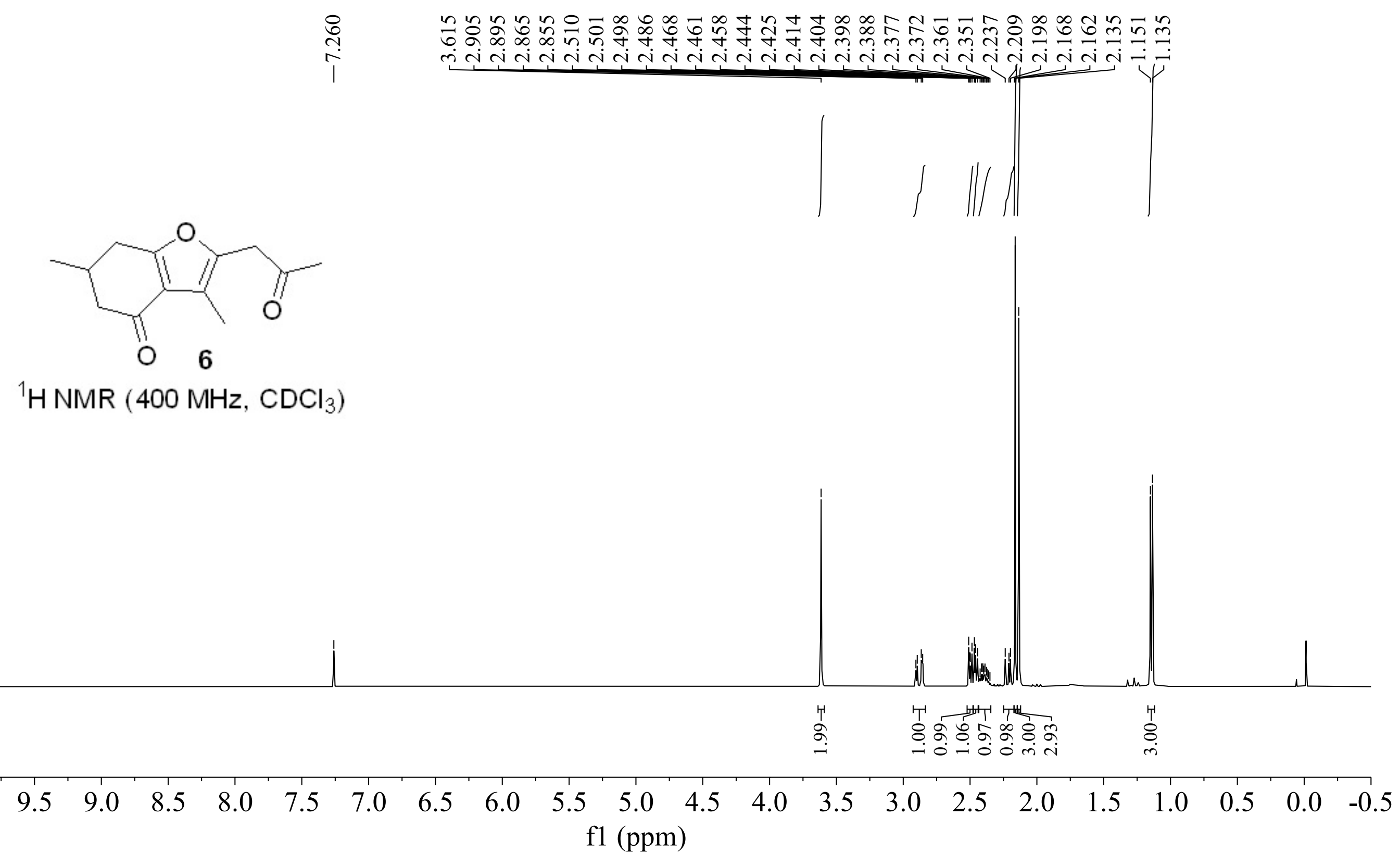




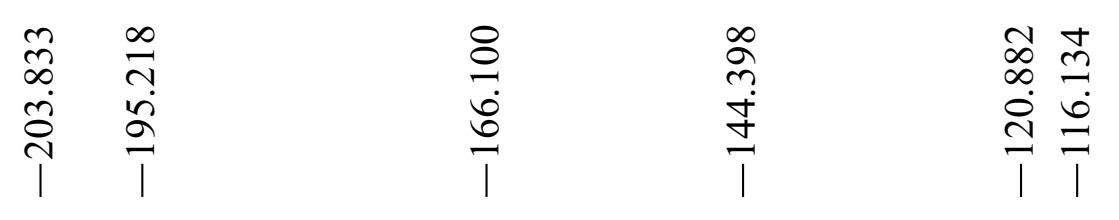

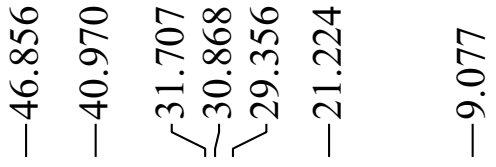

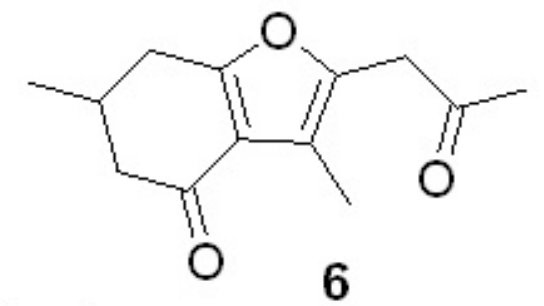

${ }^{13} \mathrm{C}\left\{{ }^{1} \mathrm{H}\right\} \mathrm{NMR}\left(101 \mathrm{MHz}, \mathrm{CDCl}_{3}\right)$

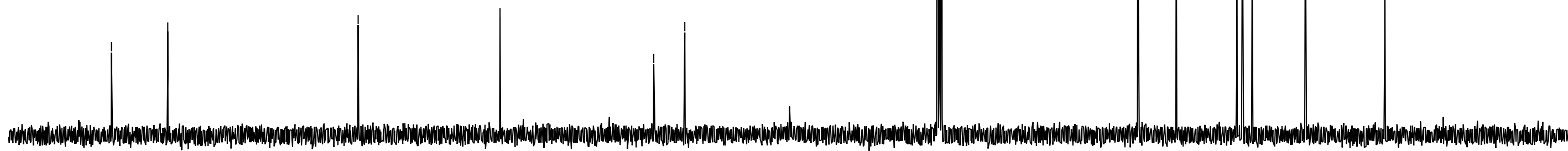

$\begin{array}{rllllllllllllllllllllll}210 & 200 & 190 & 180 & 170 & 160 & 150 & 140 & 130 & 120 & 110 & 100 & 90 & 80 & 70 & 60 & 50 & 40 & 30 & 20 & 10 & 0 & -10 \\ (10 p)\end{array}$

